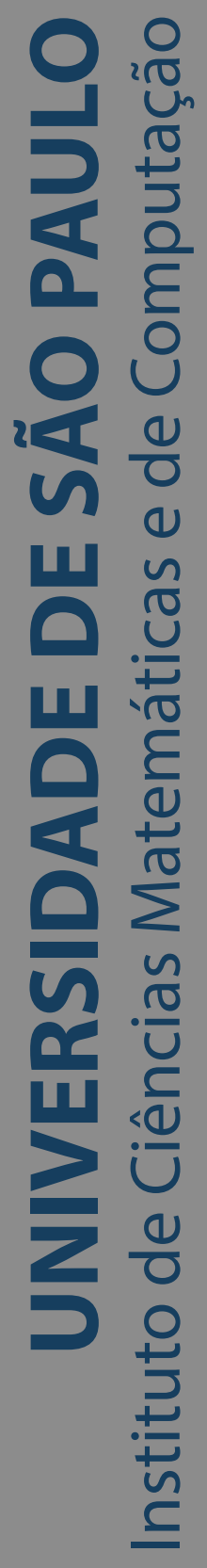

\title{
The vehicle routing problem with drones
}

\section{Joao Guilherme Cavalcanti Costa}

Dissertação de Mestrado do Programa de Pós-Graduação em Ciências de Computação e Matemática Computacional (PPG-CĆMC) 

SERVIÇO DE PÓS-GRADUAÇÃO DO ICMC-USP

Data de Depósito:

Assinatura:

\section{Joao Guilherme Cavalcanti Costa}

\section{The vehicle routing problem with drones}

Master dissertation submitted to the Institute of Mathematics and Computer Sciences - ICMC-USP, in partial fulfillment of the requirements for the degree of the Master Program in Computer Science and Computational Mathematics. FINAL VERSION

Concentration Area: Computer Science and Computational Mathematics

Advisor: Profa. Dra. Maristela Oliveira dos Santos 
Ficha catalográfica elaborada pela Biblioteca Prof. Achille Bassi e Seção Técnica de Informática, ICMC/USP, com os dados inseridos pelo(a) autor(a)

Cavalcanti Costa, Joao Guilherme The Vehicle Routing Problem with Drones / Joao Guilherme Cavalcanti Costa; orientadora Maristela Oliveira dos Santos. -- São Carlos, 2019. $124 \mathrm{p}$.

Dissertação (Mestrado - Programa de Pós-Graduação em Ciências de Computação e Matemática

Computacional) -- Instituto de Ciências Matemáticas e de Computação, Universidade de São Paulo, 2019.

1. Otimização Combinatória. 2. Algoritmo Genéticos. 3. Logística. 4. Drones. 5. Roteirização. I. Oliveira dos Santos, Maristela, orient. II. Título.

Bibliotecários responsáveis pela estrutura de catalogação da publicação de acordo com a AACR2: Gláucia Maria Saia Cristianini - CRB - 8/4938 Juliana de Souza Moraes - CRB - 8/6176 


\section{Joao Guilherme Cavalcanti Costa}

\section{O problema de roteamento de veiculos com drones}

Dissertação apresentada ao Instituto de Ciências Matemáticas e de Computação - ICMC-USP, como parte dos requisitos para obtenção do título de Mestre em Ciências - Ciências de Computação e Matemática Computacional. VERSÃO REVISADA

Área de Concentração: Ciências de Computação e Matemática Computacional

Orientadora: Profa. Dra. Maristela Oliveira dos Santos

USP - São Carlos

Maio 2019 

To Julianny,

the brightest star in the sky. 

First, I would like to thank my mother, Cristina, who always supported me in my endeavors. My family, specially my grandmother Paizinha, my father Enéas, my brother and sister-in-law, my sister and Lola, and my great aunt Marlene and uncle José, who gave me love, support and companionship throughout my whole life.

Of course, this work would not be possible without my advisor, Professor Maristela. She accepted me as her student even though I had very little experience in the field. She gave me freedom to work the way I would feel better and with a theme of my own choosing. For all the advice, insights and comprehension you gave me, thank you.

I also would like to thank the friends I made here in São Carlos, who were very crucial to keeping me sane throughout these two and a half years. To Pedro and Misael who welcomed me in a new city, I will always be grateful for that. To my flatmates, Chao, Geladinho, Eiki and Tsuruda, you guys made me feel like I belonged, like home. To Isa, Giovanni, Esther and Andre, thank you for good laughs and for showing me the city. Marye you are the best person ever, thank you for being awesome. And all other friends I made in this wonderful city, Ju, Kaori, Adriano, Check and Checkinho, Thay, Angelica, Bichão, Tiger, Nath, Maya, Minoru, Mayu and Mayu. My UFPI friends who also came to this city, Roney and JP, you guys rock. May our friendships last forever.

I would like to mention the Professor who instigated my curiosity on this field, Antonio Costa, from UFPI. I would not have done this work without your classes on Optimization and Linear Programming. For that, thank you.

Finally, a special thanks to Matheus Fernandes. You helped me a lot during my research, finding bugs in my codes and questioning my variables' names. We have been friends for years and I am truly grateful for all of it. This work is also yours.

Additionally, the authors would like to thank CNPq, CAPES, and CeMEAICEPID (FAPESP No. 2013/07375-0) for the financial support received. 

"All we have to decide is what to do with the time that is given us."

(J. R. R. Tolkien) 



\section{ABSTRACT}

COSTA, J. G. C. The vehicle routing problem with drones. 2019. 124 p. Dissertação (Mestrado em Ciências - Ciências de Computação e Matemática Computacional)

- Instituto de Ciências Matemáticas e de Computação, Universidade de São Paulo, São Carlos - SP, 2019.

In this Dissertation, the Vehicle Routing Problem with Drones (VRPD), motivated by the growing interest on Unmanned Aerial Vehicles (UAVs, or Drones) by the industry and their applications in logistics is studied. A pioneer work by (MURRAY; CHU, 2015) shows a combination between UAV and a truck to deliver products, presenting an adaptation to the Traveling Salesman Problem (TSP). After a literature review, an extension of the model from Murray and Chu (2015) we present a model for the problem with multiple vehicles. This model is developed as a Mixed Integer Linear Programming (MILP) problem and solved with the solver CPLEX. A heuristic based on a Hybrid Genetic Algorithm (HGA) is also developed and presented. Our results show that the use of drones reduces the total mileage of the trucks by a significant percentage.

Keywords: Vehicle Routing Problem, Drones, Mixed Integer Linear Programming, Hybrid Genetic Algorithm, Logistics. 



\section{RESUMO}

COSTA, J. G. C. O problema de roteamento de veiculos com drones. 2019. 124 p. Dissertação (Mestrado em Ciências - Ciências de Computação e Matemática Computacional) - Instituto de Ciências Matemáticas e de Computação, Universidade de São Paulo, São Carlos - SP, 2019.

Nessa monografia estuda-se o Problema do Roteamento de Veículos com Drones (PRVD), motivado pelo crescente interesse da indústria em Veículos Aéreos Não Tripulados (VANTs) e suas aplicações em logística. O trabalho pioneiro de (MURRAY; CHU, 2015) mostra uma combinação entre VANT e um caminhão para realização de entregas de produtos, no qual foi proposta uma adaptação do Problema do Caixeiro Viajante (PCV). Após uma revisão de literatura, apresenta-se uma extensão do modelo de Murray and Chu (2015) para o problema com múltiplos veículos. Desenvolveu-se um modelo de Programação Linear Inteira Mista que foi resolvido com o solver CPLEX. Uma heurística basead em um Algoritmo Genético Híbrido também foi desenvolvido e é apresentada. Resultados mostram que a utilização dos VANTs reduzem a quilometragem dos caminhões significativamente.

Palavras-chave: Problema do Roteamento de Veículos, VANTs, Programação Linear Inteira Mista, Algoritmo Genético Híbrido, Logística. 

Figure 1 - Drone value in different industries. . . . . . . . . . . . . . 26

Figure 2 - A comparison between a drone's route (green) and a delivery vehicle's (red) in Reykjavik. . . . . . . . . . . . . . . . . . . . . 27

Figure 3 - An instance of the VRP and one solution. . . . . . . . . . . . . 30

Figure 4 - First scenario of drone usage in logistics: delivery in downtown areas. . 40

Figure 5 - Second scenario of drone usage in logistics: delivery in rural areas . . . 41

Figure 6 - Avoided scenarios . . . . . . . . . . . . . . 50

Figure 7 - Chromosome representation. . . . . . . . . . . . 53

Figure 8 - Chromosome translation and split example. . . . . . . . . . . 54

Figure 9 - OX Crossover example. . . . . . . . . . . . . 58

Figure 10 - Flow graph for the LS process. . . . . . . . . . . . . . . . . 59

Figure 11 - Merge routes example. Here the route 726 is added to the end of the route 5314. . . . . . . . . . . . . . . . . . 60

Figure 12 - Split routes example. The first route 5314726 is split into two: 531

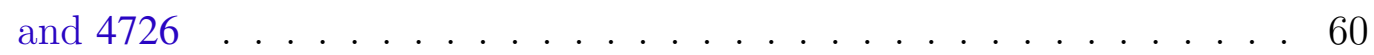

Figure 13 - Drone Local Search example, where the customer 7 is changed from truck-served to drone-served. . . . . . . . . . . . . . . . . . 61

Figure 14 - An example of the two-swap* move. . . . . . . . . . . . . . . . 61

Figure 15 - An example of the two-swap-exchange* move. . . . . . . . . . . . . . . 62

Figure 16 - An example of a two-opt* move. . . . . . . . . . . . . . . . . . 62

Figure 17 - An example of a two-opt move. . . . . . . . . . . . . . . . 63

Figure 18 - An example of the two-swap move. . . . . . . . . . . . . . . . 63

Figure 19 - An example of the two-swap-exchange move . . . . . . . . . . . . . . . 63

Figure 20 - Drone Heuristic visualization. . . . . . . . . . . . . . . . . 65

Figure 21 - Illustration of the HGA in execution. Example with instance C101 from $(\mathrm{SOLOMON}, 1987) . \ldots . \ldots . \ldots . \ldots 81$

Figure 22 - Illustration of the HGA in execution. Example with instance R206 from $($ SOLOMON, 1987). . . . . . . . . . . . . . . 82

Figure 23 - Illustration of the HGA in execution. Example with instance RC107 from $($ SOLOMON, 1987). . . . . . . . . . . . . . . . 83 

Algorithm 1 - Hybrid Genetic Algorithm structure . . . . . . . . . . . . 52

Algorithm 2 - Chromosome Split Algorithm . . . . . . . . . . . . . 55

Algorithm 3 - Sweep Algorithm . . . . . . . . . . . . . . 56

Algorithm 4 - Random Chromosome Algorithm . . . . . . . . . . . 57

Algorithm 5 - Education Step . . . . . . . . . . . . . . 59

Algorithm 6 - Drone Route Heuristic . . . . . . . . . . . . . . . . 64

Algorithm 7 - Weight Classification Algorithm . . . . . . . . . . . 68 

Table 1 - Current work on Truck and Drone problems. . . . . . . . . . . . . 37

Table 2 - General parameters for the tests. Default parameters are in bold. . . . . 69

Table 3 - Parameters for the HGA . . . . . . . . . . . . . . . 70

Table 4 - Results for the class of instances C, using the Sweep Algorithm as a starting solution. . . . . . . . . . . . . . 71

Table 5 - Results for the class of instances R, using the Sweep Algorithm as a starting solution. . . . . . . . . . . . . . . . . 72

Table 6 - Results for the class of instances RC, using the Sweep Algorithm as a starting solution. . . . . . . . . . . . . . . . 73

Table 7 - A comparison between the result of the MILP with Sweep as initial solution and the optima from the VRPTW for the class C. . . . . . . 73

Table 8 - A comparison between the result of the MILP with Sweep as initial solution and the optima from the VRPTW for the class R. . . . . . . . 74

Table 9 - A comparison between the result of the MILP with Sweep as initial solution and the optima from the VRPTW for the class RC. . . . . . . 75

Table 10 - Results for the class of instances C, using the HGA as a starting solution. 75

Table 11 - Results for the class of instances R, using the HGA as a starting solution. 76

Table 12 - Results for the class of instances RC, using the HGA as a starting solution. 77

Table 13 - A comparison between the result of the MILP with HGA as initial solution and the optima from the VRPTW for the class C. . . . . . . . 77

Table 14 - A comparison between the result of the MILP with HGA as initial solution and the optima from the VRPTW for the class R. . . . . . . . 78

Table 15 - A comparison between the result of the MILP with HGA as initial solution and the optima from the VRPTW for the class RC. . . . . . . 78

Table 16 - The effect of changes in speed in the result of the MILP + HGA. . . . . 79

Table 17 - The effect of changes in drone eligible weight in the result of the MILP + HGA. . . . . . . . . . . . . . . . . . 79

Table 18 - The HGA average for 5 runs and the best of these are compared to the VRPTW best known solutions of the class C. The average initial and final population fitness, and execution time are also shown. . . . . . . . 79

Table 19 - The HGA average for 5 runs and the best of these are compared to the VRPTW best known solutions of the class R. The average initial and final population fitness, and execution time are also shown. . . . . . . . 80 
Table 20 - The HGA average for 5 runs and the best of these are compared to the VRPTW best known solutions of the class RC. The average initial and final population fitness, and execution time are also shown. . . . . . . . 80

Table 21 - Results for the class of instances C, with 100\% drone speed and $25 \%$ drone eligible weight, using the Sweep as a starting solution. . . . . . . 97

Table 22 - Results for the class of instances C, with 100\% drone speed and $75 \%$ drone eligible weight, using the Sweep as a starting solution. . . . . . . 98

Table 23 - Results for the class of instances R, with 100\% drone speed and $25 \%$ drone eligible weight, using the Sweep as a starting solution. . . . . . . 98

Table 24 - Results for the class of instances R, with 100\% drone speed and $75 \%$ drone eligible weight, using the Sweep as a starting solution. . . . . . . 99

Table 25 - Results for the class of instances RC, with $100 \%$ drone speed and $25 \%$ drone eligible weight, using the Sweep as a starting solution. . . . . . . 99

Table 26 - Results for the class of instances RC, with 100\% drone speed and $75 \%$ drone eligible weight, using the Sweep as a starting solution. . . . . . . 100

Table 27 - Results for the class of instances C, with 50\% drone speed and 25\% drone eligible weight, using the HGA as a starting solution. . . . . . . 100

Table 28 - Results for the class of instances C, with $50 \%$ drone speed and $50 \%$ drone eligible weight, using the HGA as a starting solution. . . . . . . . 101

Table 29 - Results for the class of instances C, with 50\% drone speed and 75\% drone eligible weight, using the HGA as a starting solution. . . . . . . . 101

Table 30 - Results for the class of instances C, with $100 \%$ drone speed and $25 \%$ drone eligible weight, using the HGA as a starting solution. . . . . . . . 102

Table 31 - Results for the class of instances C, with 100\% drone speed and $75 \%$ drone eligible weight, using the HGA as a starting solution. . . . . . . . 102

Table 32 - Results for the class of instances C, with 150\% drone speed and $25 \%$ drone eligible weight, using the HGA as a starting solution. . . . . . . . 103

Table 33 - Results for the class of instances C, with $150 \%$ drone speed and $50 \%$ drone eligible weight, using the HGA as a starting solution. . . . . . . . 103

Table 34 - Results for the class of instances C, with 150\% drone speed and $75 \%$ drone eligible weight, using the HGA as a starting solution. . . . . . . . 104

Table 35 - Results for the class of instances R, with $50 \%$ drone speed and $25 \%$ drone eligible weight, using the HGA as a starting solution. . . . . . . . 104

Table 36 - Results for the class of instances R, with 50\% drone speed and 50\% drone eligible weight, using the HGA as a starting solution. . . . . . . . 105

Table 37 - Results for the class of instances R, with $50 \%$ drone speed and $75 \%$ drone eligible weight, using the HGA as a starting solution. . . . . . . . 105

Table 38 - Results for the class of instances R, with $100 \%$ drone speed and $25 \%$ drone eligible weight, using the HGA as a starting solution. . . . . . . 106 
Table 39 - Results for the class of instances R, with 100\% drone speed and $75 \%$ drone eligible weight, using the HGA as a starting solution. . . . . . . . 106

Table 40 - Results for the class of instances R, with 150\% drone speed and 25\% drone eligible weight, using the HGA as a starting solution. . . . . . . . 107

Table 41 - Results for the class of instances R, with 150\% drone speed and 50\% drone eligible weight, using the HGA as a starting solution. . . . . . . . 107

Table 42 - Results for the class of instances R, with 150\% drone speed and $75 \%$ drone eligible weight, using the HGA as a starting solution. . . . . . . . 108

Table 43 - Results for the class of instances RC, with 50\% drone speed and 25\% drone eligible weight, using the HGA as a starting solution. . . . . . . . 108

Table 44 - Results for the class of instances RC, with 50\% drone speed and 50\% drone eligible weight, using the HGA as a starting solution. . . . . . . . 109

Table 45 - Results for the class of instances RC, with $50 \%$ drone speed and $75 \%$ drone eligible weight, using the HGA as a starting solution. . . . . . . . 109

Table 46 - Results for the class of instances RC, with 100\% drone speed and $25 \%$ drone eligible weight, using the HGA as a starting solution. . . . . . . . 110

Table 47 - Results for the class of instances RC, with 100\% drone speed and $75 \%$ drone eligible weight, using the HGA as a starting solution. . . . . . . . 110

Table 48 - Results for the class of instances RC, with $150 \%$ drone speed and $25 \%$ drone eligible weight, using the HGA as a starting solution. . . . . . . . 111

Table 49 - Results for the class of instances RC, with $150 \%$ drone speed and $50 \%$ drone eligible weight, using the HGA as a starting solution. . . . . . . . 111

Table 50 - Results for the class of instances RC, with $150 \%$ drone speed and $75 \%$ drone eligible weight, using the HGA as a starting solution. . . . . . . . 112 



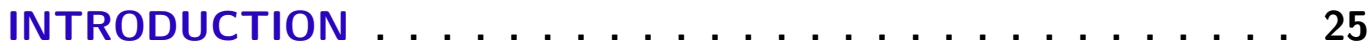

LITERATURE REVIEW . . . . . . . . . . . . . . . . . . . . 29

PROBLEM DEFINITION AND MODEL . . . . . . . . . . . . 39

3.4 The VRPD Mixed-Integer Linear Programming Model . . . . . . . . 43

4.2.1 Split Procedure . . . . . . . . . . . . . . . . 53

$4.3 \quad$ Initial Population . . . . . . . . . . . . . . . . 55

$4.4 \quad$ Crossover and Selector Operators . . . . . . . . . . . . . 57

$4.5 \quad$ Education . . . . . . . . . . . . . . . . . . . . 58

4.5.1 Drone Route Method . . . . . . . . . . . . . . . . . . . . . . . 62



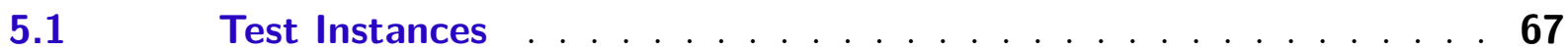

5.1.1 Overall Parameters . . . . . . . . . . . . . . . . . . . . . 68

5.1.2 CPLEX Parameters . . . . . . . . . . . . . . . . . 69

5.1.3 Hybrid Genetic Parameters . . . . . . . . . . . . . . . . . . 70

$5.2 \quad$ MILP Results . . . . . . . . . . . . . . . . . . 70

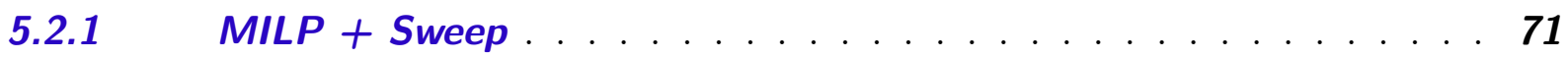

$5.2 .2 \quad M I L P+H G A \ldots \ldots \ldots 72$






BIBLIOGRAPHY ......................... 87

ANNEX A $\quad$ MILP MODEL FOR FSTSP . . . . . . . . 93

ANNEX B ADDITIONAL RESULTS AND TABLES . . . . . . . . 97



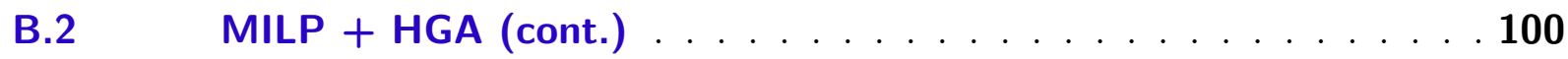

ANNEX C A MIXED INTEGER LINEAR PROGRAMMING MODEL

FOR THE DRONE-ASSISTED TSP . . . . . . . . . 113 
CHAPTER

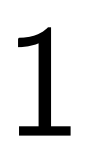

INTRODUCTION

Modern customers' eagerness to receive everything faster, whether it being information through the Internet, or services in general, has compelled the market and industry to invest in technologies to meet these customers new demands. One of these new technologies is civil unmanned aerial vehicles (UAV), which are commonly known as drones.

Recent advances in drone technology allow applications that were real only in science fiction to become a reality. Including video capture for personal use, surveillance, inspection of cables and structures, or even using drones as auxiliary video capture units in the Winter Olympics 2018 (INTEL, 2018). The drones can also be used for saving lives. Drones are being used for disaster reliefs, where they can transport water, blood, medicine and equipment for rescue teams (MEIER, 2018),(CHOWDHURY et al., 2017), (PULVER; WEI, 2018); or even organ deliveries (SEILER, 2019).

The focus of this work, however, is the use of drones in the transportation or in delivery business, which can include medical applications, but are not limited to it. Some companies are interested in applying drones to their transportation markets, such as DHL (KUHLMANN, 2016), UPS (LITHIA, 2017), Amazon (CBS, 2013), Dominos (SCHOLZ, 2016), and Google (XCOMPANY, 2017). Several small or test applications are already being developed as well: 7-Eleven is testing small products being delivered by autonomous drones in America (SHAW, 2016); DHL, in Germany, is delivering pharmaceutics to an island (DHL, 2014); Zipline's drones are being used to transport blood and medicine to remote locations in Rwanda (SIMMONS, 2016).

All these applications under development or even in execution show that the drone industry is promising. Market trends reported by the Business Insider magazine suggest a growth in drone sales that will reach $\$ 12$ billion dollars by 2021 (MEOLA, 2017). To illustrate this growth Figure 1 shows drone values in different industries in 2015, where, for 
example, $\$ 13$ billion dollars were invested on drone transportation solutions. Apart from delivery speed, accessibility is a strong appeal for the use of drones, which helps to justify the investments. For example: locations that are hard to access by regular vehicles because they lack pavemented roads (or have no roads at all); islands or other isolated areas (due to landslides, fires and other disasters); highly dense urban areas, where heavy traffic or vehicle limitations are present; or even rural areas, where the distance between residences can be significant. This way, new markets (more clients) can be reached, and/or time (and therefore money) can be saved. Another recurrent concern in the transportation industry is the emission of greenhouse gases, which adds an extra incentive to use drones. As most drones are battery-powered, they can lower up to $54 \%$ of emissions (STOLAROFF; SAMARAS, 2018).

Figure 1 - Drone value in different industries.

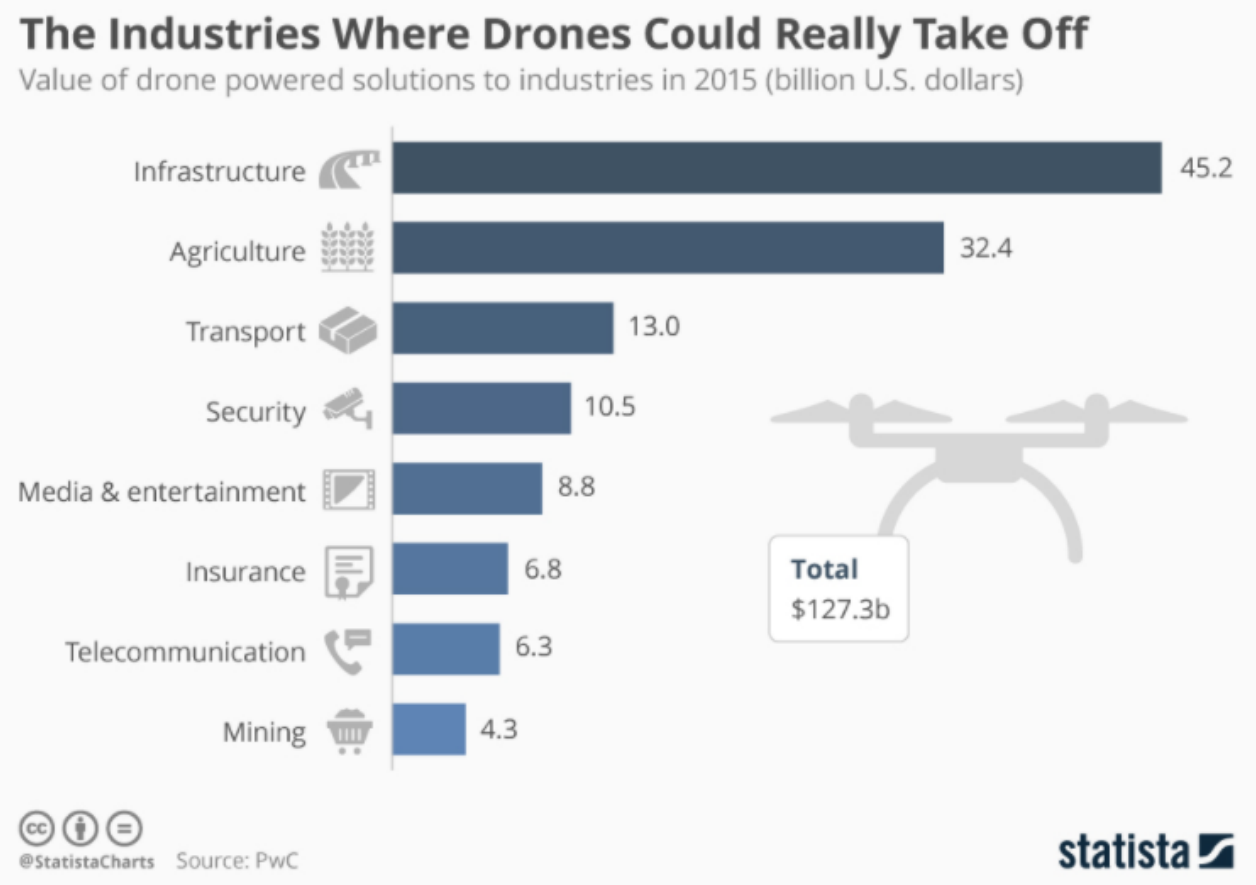

Source: (STOLAROFF; SAMARAS, 2018)

A successful application with drone in transportation and delivery is the Icelandic company AHA, which is using Flytrex's drones to deliver products to customers who are located at the other side of a bay in Reykjavík (O'KANE, 2017). As seen in Figure 2, the distance reduced by the use of the drones is noticeable. This smaller route cuts delivery times and costs significantly. The company employs drones to fly over the bay and a deliveryman would wait on the other side collecting the drone's cargo. Then, the deliveryman would serve the customers on that side of the bay. We explored this idea of a drone transporting parcels to a deliveryman at another location in a paper that was published in a 
national operations research conference (COSTA et al., 2018). The paper can be seen in the Appendix C.

Figure 2 - A comparison between a drone's route (green) and a delivery vehicle's (red) in Reykjavik.

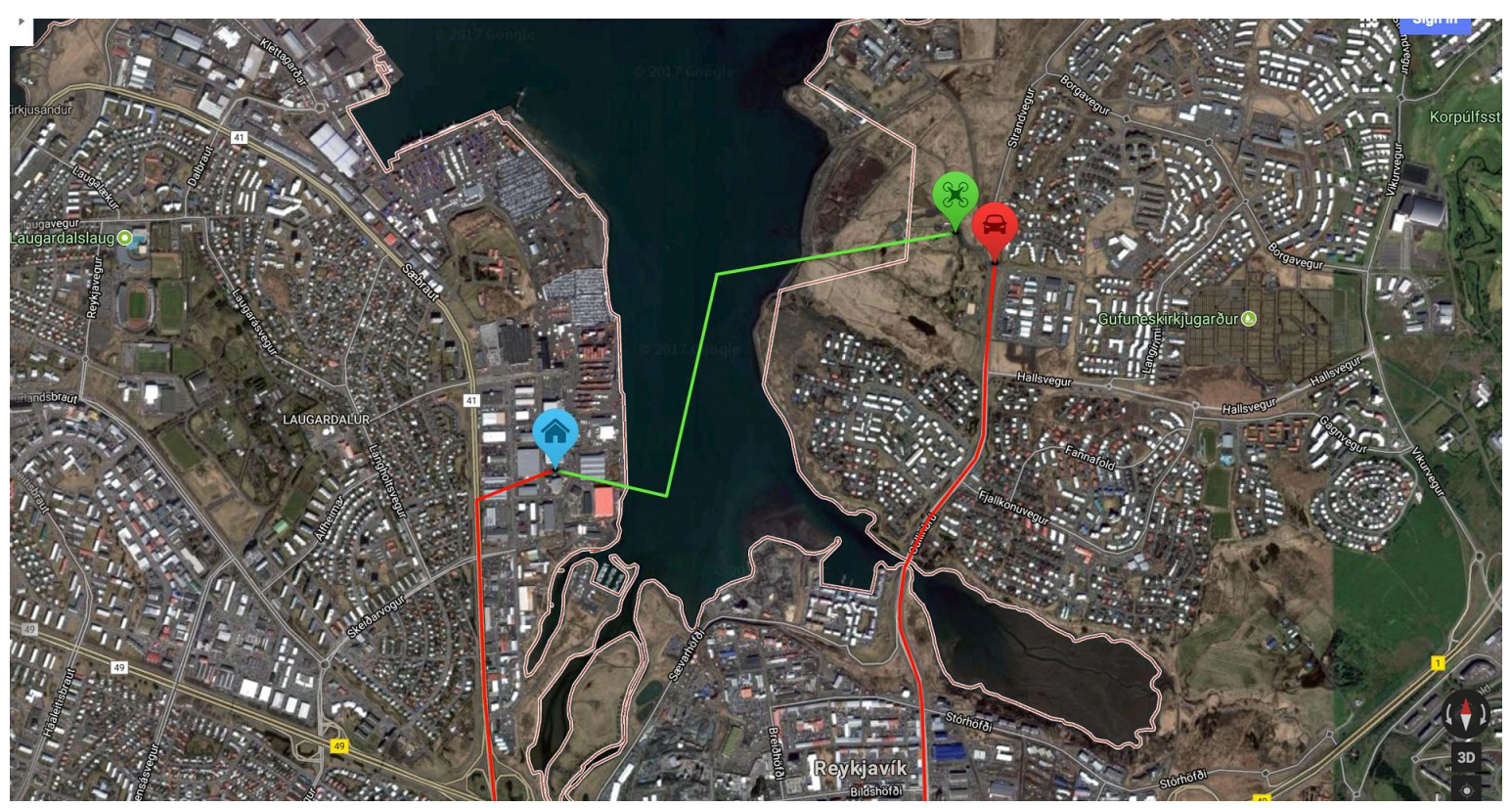

Source: Flytrex, found in (GILCHRIST, 2017).

Although the use of drones seems to be very promising, many challenges arise in real applications, such as the legislation on drone usage (privacy issues, congested airspace and threat to other aircraft, and even safety issues), the engineering difficulty for autonomous flight, the logistics implications of using vehicles with spatial and weight limitations, and the current batteries' duration. DHL has written a report on these problems and the expectations for drones in (KüCKELHAUS; NIEZGODA; ENDRIß, 2014). Some papers have explored a couple of these adversities, such as (HONG; KUBY; MURRAY, 2018) with recharging stations, and (EREN; ÇELIK, 2018) with risk assessment. Given the current adversities and their implication has lead to manufacturers, logistics and transportation companies to research and invest into solving them.

This dissertation focuses on the logistics aspect, creating solutions methods for drone delivery. As most companies are in a stage of research and legislation challenges, some assumptions have to be made on how applications are going to work, and, therefore, results may be overly theoretical.

One of the main problems in logistics is defining routes for vehicles. The classical Traveling Salesman Problem (TSP) is the basic problem in the field. When several vehicles are involved, and adding a limited capacity, an extension of the TSP is born as the Vehicle Routing Problem (VRP). The addition of Drones in this process is the Vehicle Routing 
Problem with Drones (VRPD). The main differences from VRP using drones to the classic VRP, or even to other variants, are: the reduced capacity, the trend being to deliver one package per flight; the distance covered (or flight endurance) when compared to other vehicles is very small; and not all deliveries or customers are able to be served with a drone, either because of weight or lack of landing sites.

An alternative, so as to reduce some of these difficulties and while benefiting from the advantages offered by drones, is the use of trucks (or other medium to large vehicles) with a drone in tandem. This way the drone flies from one point (a customer served by the truck) to the client it has to deliver to, then meets the truck in another customer's location. Thus, long distance trips can be executed by the truck (as well delivering packages which are not drone-eligible) and short distance trips (or areas with heavy traffic) can be covered by the drone, especially in context of last-mile delivery. This idea is being tested by UPS (LITHIA, 2017) and was first introduced in the scientific field (as far as the authors are aware) by (MURRAY; CHU, 2015), where only one truck-drone pair is used. Their work will be further explored in Chapter 2. On the other hand, in several real-life applications, using only one truck (and one drone) is not enough. Due to the large number of clients and/or the limited time for delivery, most logistic companies use several vehicles to fulfill their demands. The VRP, which is used for such cases, is not covered by the aforementioned model. The use of this truck-drone system as a VRP is explored in (WANG; POIKONEN; GOLDEN, 2016), where the authors provide mathematical proof that the time for delivery is indeed reduced when using drones. However, the authors do not provide any mathematical model nor solution method for the problem.

Hence, the purpose of this dissertation is to provide a mathematical Mixed Integer Linear Problem (MILP) model based on the formulation from (MURRAY; CHU, 2015), extending it to a VRPD, adding constraints and/or variables and adapting them to fit the original model to the idea of using several truck-drone pairs. A solution method based on a Hybrid Genetic Algorithm (HGA) in combination with Local Search (LS) is also introduced as an realistic (time-wise) technique to solve the VRPD.

It is important to note that this work, despite being inspired by the UAV technology and its applications, can also be applied for other types of autonomous vehicles such as wheeled robots (which can also be referred as drones), since the principles of other autonomy vehicles are very similar to the ones applied to UAVs. The differences would mainly be about parameters adjustment.

The following chapters are divided as follows: in Chapter 2 a literature review on the VRP and drone problems related to this work is presented; Chapter 3 presents scenarios for the problem and its description as well as the MILP model; Chapter 4 presents our HGA and the LS used; The results are discussed in Chapter 5; Finally, we summarize the work in Chapter 6, as well as present some research perspectives. 
CHAPTER

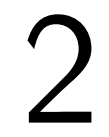

2

\section{LITERATURE REVIEW}

In this chapter, a preliminary review of the literature on the subject of the Vehicle Routing Problem with Drones is presented. First, the origins of the VRP and some types of VRP are given to provide some context. Afterwards, related TSP and VRP work in logistics with drones are addressed. Finally, a comparison of this work with all the other works presented is shown.

\subsection{An Introduction to VRP}

Formally, the Vehicle Routing Problem (VRP) in its classical formulation is defined by a set of $m$ vehicles with capacity $Q$ each, which have to visit a set of customers with demand $q_{i}$ (being either delivery or pick-up). The world model is represented by a directed graph $G=(V, A)$, where the Vertices $V=\{0,1, \ldots, n\}$ are the customers (with 0 being the depot), and the $\operatorname{Arcs} A=\{(i, j): i, j \in V, i \neq j\}$ being the distance from node $i$ to node $j$ (in time or distance units). The VRP is a generalization of the Traveling Salesman Problem (TSP) with several vehicles and limited capacity. In the TSP a single salesman wants to visit every city (from a set of cities) in such a way that he visits all cities only once, reducing the total distance traveled. In the VRP, however, $m$ routes are computed, one for each vehicle, every one of them starting and ending in the depot, with every customer being visited exactly once, and no route demand exceeds the capacity $Q$, i.e. to find the shortest feasible routes. Both the TSP and the VRP are of NP-Hard complexity, and are therefore very costly to solve (LENSTRA; KAN, 1981). Figure 3 shows a visualization of the problem.

The first work published for the VRP was presented by (DANTZIG; RAMSER, 1959). The truck dispatching problem, as it is called in their work, tries to find an optimal solution to a gasoline delivery service, from one single distribution center (a depot), containing several trucks and customers. The objective is to find the minimal route for 
Figure 3 - An instance of the VRP and one solution.
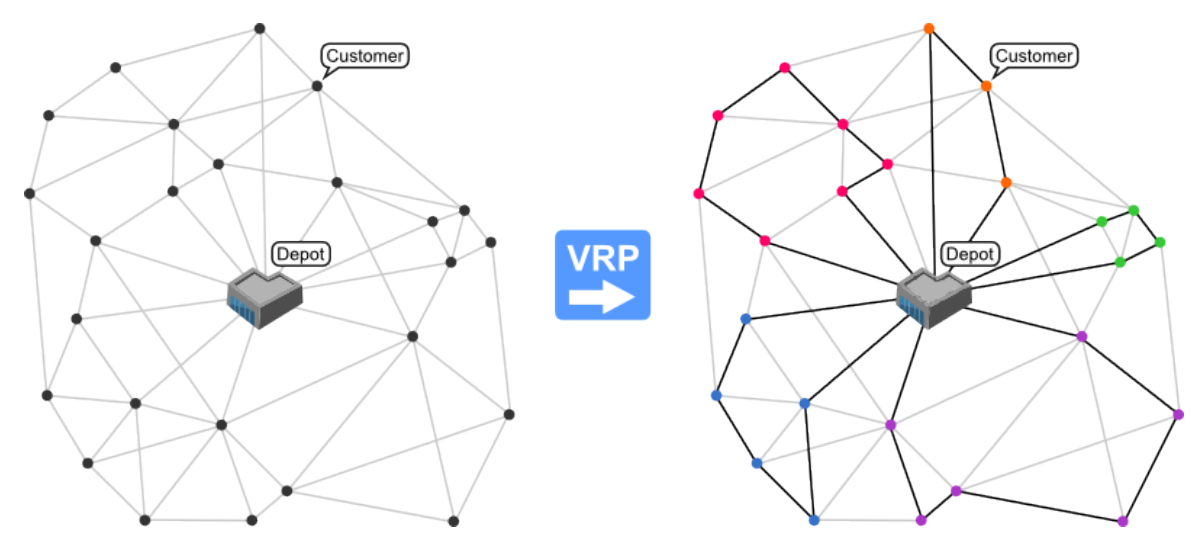

Source: (NETWORKING; OPTIMIZATION, 2013)

every truck in a way where each customer is visited exactly once, and their demand is satisfied. The trucks also present the same capacity (homogeneous fleet). Later, (CLARKE; WRIGHT, 1964) wrote a generalization of Dantzig and Ramser (1959) as a linear optimization problem, and proposed the Savings Algorithm heuristic to solve it.

Both of these works were later specified as a Capacitated VRP, since they acknowledge that the vehicles have a transportation capacity. As the VRP grew in popularity the following decades, several variations were introduced to represent more realistic situations. For example, the addition of time constraints was introduced as VRP with Time Windows (VRPTW), where customers have a specific time window they can be served. Another variant is the pick-up and delivery, where cargo is to either be picked-up or delivered during the course, taking into account the order in which the customers will be visited so the vehicle can be more efficiently filled or emptied. More variants are related to the dynamism of the problem, whether by customers being unknown before execution or following stochastic demands, or even the travel times, for example, taking into account traffic or detours. For more on the VRP and its variations check (BRAEKERS; RAMAEKERS; NIEUWENHUYSE, 2016), (LAPORTE, 2009), (EKSIOGLU; VURAL; REISMAN, 2009).

In the classical literature for the VRP, the problem that is comparable to the approach of truck and drone (VRPD) is the VRP with multiple deliverymen (VRPMD), with only one deliveryman, since delivery drones have a relatively large size, so it is impractical to fit more than one in a single truck. In the VRPMD a vehicle is driven to a parking location, and then the servicemen (deliverymen) walk to the customers, returning afterwards to the parked vehicle to continue to the next parking point (for more on the VRPMD read (PUREZA; MORABITO; REIMANN, 2012)). However, this problem is not similar enough to VRPD, as the drones have batteries which need to be taken into account and the truck does not need to stay parked, waiting for the drone. The VRPD 
could also be characterized as a Green Vehicle Routing Problem (GVRP), as it utilizes a sustainable transportation method (LIN et al., 2014) in the form of a battery-powered drone and implicitly reduces the total mileage covered by the truck. In the next section, works related to that of the VRPD are presented.

\subsection{Use of Drones in Logistics}

Using drones for package delivery is a very recent development, and there was a boom in the last two years (2017-2018) of several works in literature being published. (MURRAY; CHU, 2015) wrote one of the first papers on delivering with drones (using the UAV notation), called the Flying Sidekick Traveling Salesman Problem (FSTSP), referring to the truck with its UAV partner. They present two different Mixed Integer Linear Programming (MILP) approaches for two types of delivery: the first one is a combination of one truck and one UAV delivering to customers outside the flying range from the depot, and a second one where several UAVs deliver directly from the depot and a truck delivers to customers who are out of range (called the PDSTSP, or Parallel Drone Scheduling TSP); in both cases they seek to minimize the time the last vehicle (UAV or truck) returns to the depot. Their work introduces the idea of having two different types of customers, those to which an UAV can deliver, and those to which it cannot, either because of the product's weight, because the site does not allow clear landing, and others. Murray and Chu (2015) solve the first problem (truck and an UAV) with a TSP heuristic that finds a route for the truck, which gets improved by checking whether delivering to a client with an UAV is viable (reduces total delivery time) and then adjusting the truck's route accordingly. The second problem is solved by initially using a TSP heuristic for the truck, including all customers that are out of range and whose demand is not UAVsuitable, and the remaining customers are scheduled to the UAVs by using a parallel identical machine scheduling problem heuristic, where a customer is a job, the flight time is the processing time, and the objective is to have a minimal makespan. That initial solution is improved by checking if swapping the customers from one route to another is viable to the time cost (of course, respecting the UAV-suitable restrictions). Their results, which compare a truck-only TSP with their models, show an improvement to the solutions. The model for the FSTSP, which is the base for the model in this Dissertation, is available in Appendix A.

The study of (AGATZ; BOUMAN; SCHMIDT, 2015) is very similar to the one of (MURRAY; CHU, 2015). They named it as the Traveling Salesman Problem with Drone (TSP-D) and developed an Integer Programming (IP) model based on operations for the problem. They also propose a heuristic approach to solve it where they find a truck-only route, and then improve it by adding customers to the drone route, based on a fast greedy heuristic and an exact partitioning algorithm. 
(HA et al., 2015) propose two heuristics to solve the TSP-D, both based on clustering techniques to find the customers which will be served by the drone. They compare their results with the heuristic proposed in the FSTSP (MURRAY; CHU, 2015), and achieve better results in some test cases, but most of their results do not.

(FERRANDEZ et al., 2016) utilize a combination of a truck and one or more drones. They use a K-Means algorithm clustering the clients to find parking points where a truck could stop and send the drones in tandem to deliver packages. They then apply a genetic algorithm to solve the TSP between parking spots. The authors show results in terms of time and energy, where the first one is improved when compared to the standalone TSP, but is largely affected by the speed and/or the number of drones, and the second one vastly surpasses the first one when compared to the truck alone, claiming the drones are more efficient energy-wise. The algorithm developed was able to solve instances of more than 200 customers, in around two minutes.

(DORLING et al., 2017) elaborate a VRP using drones only, where one drone can deliver to multiple customers. From a starting depot, the drones are routed to serve all customers and they are allowed to return to the depot multiple times. Dorling et al. (2017) write an MILP to the problem, in which not only routing and re-routing are done, but also each drone's battery weight and consumption are measured, that way they can optimize the weight and the number of routes a drone can fly, with a single charge. They propose this model either to minimize delivery time or to minimize the number of drones used and energy consumption. They solve the model with a simulated annealing heuristic. They also did a linearization of the power consumption equation for drones, and a practical test for the average voltage, current and power consumed in some flight modes.

(WANG; POIKONEN; GOLDEN, 2016) write an analysis of worst-case scenarios for the Vehicle Routing Problem with Drones (VRPD), and are the first to use this terminology. The paper is for theoretical results only. The authors show mathematical relations for different scenarios of drone usage in combination with trucks, where each drone carries a single package, where the goal is to minimize time of completion, proving both upper and lower bounds for all cases, varying the number of trucks, the speed of the drones and the number of drones in each truck. The work of (WANG; POIKONEN; GOLDEN, 2016) is very relevant to any posterior work in the VRPD, since the results show the relation between the regular TSP or VRP with the VRPD. For example, one particular case with $m$ trucks and 2 drones each will have the completion time reduced by at most $75 \%$ when compared to a VRP also with $m$ trucks, considering the drones travel $50 \%$ faster than the truck.

The authors from (WANG; POIKONEN; GOLDEN, 2016) also wrote another work on the subject, (POIKONEN; WANG; GOLDEN, 2017), where they expand their scenarios to more realistic situations with more mathematical proof, taking into account 
the battery life, different metrics for drones and trucks, and the costs of utilizing drones. They also provide proof that the Close-Enough Vehicle Routing Problem (CEVRP), where trucks do not need to visit exactly the location of a client, but instead must be within some distance $R_{i}>0$, is a bound to VRPD.

(PUGLIESE; GUERRIERO, 2017) propose a MILP model for the VDRPTW, Vehicle and Drone Routing Problem with Time Windows, where several trucks with drones in tandem are used to perform parcel deliveries. Their model has similarities with the one of (MURRAY; CHU, 2015), whereas a drone can only deliver one package before returning to its truck, at a client location, and the truck will make deliveries whilst the drone is flying. The synchronization process is similar as well, although they disregard time to set-up launch or landing, and take into account the service time. The main difference between the models, besides several vehicles and the set-up time, and therefore their main contribution, is that (PUGLIESE; GUERRIERO, 2017) also add time windows for customers visits, the trucks' capacity and the objective function, which aims to minimize the total transportation cost for both drones and trucks. They test the model for small samples ( 5 and 10 customers, and a percentage of that is drone-eligible) with 2 vehicles. Their results, however, show that the use of drones is very costly even in those small instances. For example, with the same cost of transportation for trucks and drones, the model resulted in no drones being used in the optimal solution. The drones are used only when the transportation cost of a truck is more expensive. When comparing operational time, however, the drones do reduce the completion time, as expected and as pointed in the work of (WANG; POIKONEN; GOLDEN, 2016).

(SCHERMER; MOEINI; WENDT, 2018) propose two heuristics to solve the VRPD, and test them using 750 large-scale instances, from the TSPLIB dataset ${ }^{1}$. Their assumptions on how the drone and truck combination work are based on (WANG; POIKONEN; GOLDEN, 2016). The two heuristics developed are based on a route-first cluster-second approach, where a single route is created with a nearest neighbor heuristic, the route is equally divided between the $m$ trucks and are then improved. In the first heuristic they improve the trucks route before adding drones. In the second one, the drones are considered from the beginning. On their tests, the authors show that the first heuristic presents better results when compared to the second one, concluding that finding better VRP routes before adding the drones will result in better VRPD routes.

(FREITAS; PENNA, 2018) present a Randomized Variable Neighborhood Descent (RVND) heuristic to solve the flying sidekick traveling salesman problem (FSTSP) (MURRAY; CHU, 2015). On the proposed heuristics, first they solve the regular TSP optimally with the solver Concorde plus CPLEX. Then, a initial solution for the FSTSP is created by removing customers from the truck's route and adding them to the drone's route.

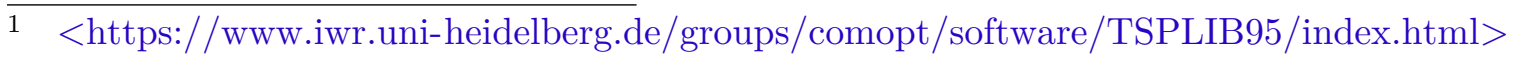


Finally, this route is improved with the RVND where, similarly to the VND, from the starting route, neighbor routes are explored and the best one is set as the new route and then moving to a new neighborhood, the difference being that there is a shuffle in the neighborhoods in the RVND. The neighborhoods utilized in their work are based on: changing one truck customer location; permutation between two truck customers; swapping two consecutive customers and an additional one; 2-OPT; and, one customer from the truck's route is relocated to the drone's route. To test their results, the authors utilize 11 instances of the TSPlib dataset, containing between 51 to 100 nodes. Although in the best cases the GAP between the regular TSP and their heuristic is an average of $7.2 \%$ improvement, the overall gap considering the 10 runs is only $0.057 \%$, for about 1 second of execution time. The best result, improved delivery time by almost $20 \%$.

(LUO; LIU; SHI, 2017) write a paper on Two-echelon Cooperated Routing Problem where, similarly to the TSPD, they use one ground vehicle and one UAV. However, the problem approach is quite different, as all customers are served by the UAV, which can serve multiple customers before returning to the vehicle. Additionally, the UAV can only rendezvous with the vehicle in predetermined parking spots. They solve this problem by proposing an IP formulation, and two constructive heuristics. The first one builds a single route with all nodes and splits it by adding the parking points, while the second one builds a route for the vehicle first (with the parking spots) and them assigns the UAV routes based on that. They create one dataset with up to 200 customers and 80 parking spots, randomly distributed, resulting in 600 instances. They compare the IP formulation with the heuristics in the 30 instances that the model could solve (with times of up to 12000s), and prove that the heuristics are competitive. For larger instances, they compare both heuristics with each other, where the first one is more accurate, but the second one is faster.

(CARLSSON; SONG, 2018) aims to find how efficient is the FSTSP with a mathematical approach. The authors use asymptotic theoretical analysis in the Euclidean plane, with computational experiments, to demonstrate that the use of the UAV will improve the efficiency of deliveries on a proportion equal to the square root of the ratio between the truck's speed and the UAV's speed.

(BOUMAN; AGATZ; SCHMIDT, 2017) propose an exact approach for the TSP-D by using Dynamic Programming (DP). To summarize, the DP consists of three main steps: enumerating all shortest paths for the truck; a combination of truck paths with the drone nodes; and the computation of the optimal path given previous steps. The authors also apply an $A^{*}$ algorithm to guide the proposed DP, to try to solve it faster. The results show that the DP does succeed in finding exact solutions faster than the IP model of the same authors Agatz, Bouman and Schmidt (2015), and that the addition of the $A^{*}$ technique also helps solve some larger instances, while also saving CPU time. 
(CHUNG, 2018) propose a heuristic method for the FSTSP, here called Truck and Drone Parcel Delivery Problem (TDRP). The heuristic, named Two Step Ahead Tour Growing Heuristic, builds the truck route constructively based on a saving heuristics. The savings is built for all combination of three customers, with one of them being served by the drone. Then, starting from the depot, the least costing route is added to the current one. The author create an small toy problem with 9 customers to test this heuristic, saving up to $33 \%$ in completion time.

(HAM, 2018) propose an extension to the PDSTSP (where drones serve directly from the depot and go back) containing: pick-up and delivery, multiple trips, multiple depots, and time-windows. The drones can leave the depot with one parcel, drop it at the desired customer location, and then fly to another customer to perform a parcel pickup, then returning to one of the depots. The trucks will work similarly, however they can serve (deliver or pick-up) several customers before returning. The author utilize two distinct Constraint Programming models to solve the problem. He tests the model with instances of 20,50 and 100 customers, finding optimal values to most instances with 20-50 customers within 300s of execution using IBM ILOG CP OPTIMIZER.

The authors of Ha et al. (2015) write another paper on TSP-D, (HA et al., 2018), where they apply two new heuristics and a new MILP model for the problem, aiming to minimize operational costs with added waiting time variables (which accounts for the time the truck or the drone have to wait for each other). The first heuristic is a GRASP with adapted local search algorithms to better fit the drone usage. The second heuristic is based on (MURRAY; CHU, 2015), consisting of constructing a truck route and adding local search to find drone routes. The authors test their solutions in instances of 10, 50 and 100 customers, running a total of 1080 tests. The exact solution can only solve the ones with 10 customers within $1 h$, and therefore cannot be compared with the heuristics for other instances. In those which can, the GRASP performs a lot better than the second heuristic, finding optimal solutions in all cases. For the larger instances, the GRASP still outperforms the other, but the average execution time is higher. When compared to the heuristic of (MURRAY; CHU, 2015), the GRASP also finds better or equal solutions for most compared cases.

(CHANG; LEE, 2018) solve the TSP-D with a nonlinear programming model approach. They find clusters of customers using K-means, and a TSP where the trucks visits all clusters centers to where the drones will fly from. They also shift the clusters centers to minimize the truck distance, yet without any customer getting out of range for the drones and compare the two models. They test the models with random instances ranging from 10 to 100 customers, showing that by shifting the clusters centers, which increases the use of drones, is more effective since in their experiments the drones are faster than the trucks. 
The dissertation of (PONZA, 2016) does an study on the FSTSP. She analyzes the problem numerically, investigating the trade-offs of faster drones or drones with longer flight autonomy. She also develops a Simulated Annealing (SA) heuristic to solve the problem. To test the algorithm and to collect results, she develops her own instances ranging from 5 to 200 customers (not regularly). She compares the SA result to the regular TSP, and finds the drones improve all instances when comparing distance. She also shows that a faster drone is actually more influential than one that flies for longer periods, even though both are not that significant. The first case improves about at average $3.5 \%$ when improving the speed by $50 \%$, and the second case average around $2.5 \%$ when the endurance is increased in approximately $60 \%$. However, these tests are made only to a very small sample, and may lack statistical significance.

(MATHEW; SMITH; WASLANDER, 2015) use a directed graph in this problem similar to the TSP-D. The difference here is that the truck follow the street network, while the drone fly directly to the customer. They formulate the problem as an optimal path planning problem, and solve it by using a novel reduction to the Generalized TSP. The authors prove that the problem is of NP-hardness complexity.

Similar to the last paper, (OTHMAN et al., 2017) propose a problem more similar to the FSTSP, where only the drones deliver the parcels, but with a pre-determined route for the truck. They model the problem in a graph of special structure. The authors also prove that this problem is of NP-hardness complexity. They solve it by finding minimumcost paths in the special graph.

(ULMER; THOMAS, 2018) write a paper on the VRP with drones for Same Day Delivery (SDD), and heterogeneous vehicles, with stochastic demands. They use a policy function approximation method to solve the problem, and find that the use of drones may reduce operational costs.

\subsection{Literature Summary}

Table 1 summarizes the works mentioned and identifies their contribution, whether it is a truck + drone approach, what kind of exact method (if any) and type of heuristic are used. Although there are a MILP formulation and some variations have a non-exact solution method for the VRPD, we deem our work is more complete and more competitive than the ones listed here. It is also important to note that not all work related to the use of drones in logistics are listed, since there is several already published. However, we tried to list the most relevant and the most related to this work. In the next chapter 3, we present the MILP formulation and the overall definition of the VRPD. After that, Chapter 4 present our HGA and LS procedures. 
Table 1 - Current work on Truck and Drone problems.

\begin{tabular}{|c|c|c|c|c|}
\hline Work & $\begin{array}{c}\text { Truck } \\
+ \\
\text { Drone }\end{array}$ & Issue & $\begin{array}{l}\text { Exact } \\
\text { Method }\end{array}$ & Heuristic \\
\hline (MURRAY; CHU, 2015) & $\checkmark$ & TSP & MILP & $\begin{array}{l}\text { Construct }+ \\
\text { Savings }\end{array}$ \\
\hline (AGATZ; BOUMAN; SCHMIDT, 2015) & $\checkmark$ & TSP & IP & $\begin{array}{c}\text { Greedy }+ \\
\text { Partitioning }\end{array}$ \\
\hline (HA et al., 2015) & $\checkmark$ & TSP & - & Clustering \\
\hline (FERRANDEZ et al., 2016) & $\checkmark$ & TSP & - & $\begin{array}{l}\text { K-Means + } \\
\text { Genetic }\end{array}$ \\
\hline (DORLING et al., 2017) & - & VRP & MILP & Simulated Annealing \\
\hline (WANG; POIKONEN; GOLDEN, 2016) & $\checkmark$ & VRP & - & - \\
\hline (POIKONEN; WANG; GOLDEN, 2017) & $\checkmark$ & VRP & - & - \\
\hline (PUGLIESE; GUERRIERO, 2017) & $\checkmark$ & VRP & MILP & - \\
\hline (SCHERMER; MOEINI; WENDT, 2018) & $\checkmark$ & VRP & - & $\begin{array}{c}\text { Route-first } \\
\text { Cluster-second }\end{array}$ \\
\hline (FREITAS; PENNA, 2018) & $\checkmark$ & TSP & - & $\begin{array}{c}\text { Random Variable } \\
\text { Neighborhood Descent }\end{array}$ \\
\hline (LUO; LIU; SHI, 2017) & $\checkmark$ & TSP & IP & $\begin{array}{c}\text { Two Constructive } \\
\text { Heuristics }\end{array}$ \\
\hline (CARLSSON; SONG, 2018) & $\checkmark$ & TSP & - & - \\
\hline (BOUMAN; AGATZ; SCHMIDT, 2017) & $\checkmark$ & TSP & $\overline{\mathrm{DP}}$ & - \\
\hline$(\mathrm{CHUNG}, 2018)$ & $\checkmark$ & TSP & - & Savings-based \\
\hline (HAM, 2018) & $\checkmark$ & mTSP & $\mathrm{CP}$ & - \\
\hline (HA et al., 2018) & $\checkmark$ & TSP & MILP & $\begin{array}{c}\text { GRASP } \\
\text { and } \\
\text { Constructive + } \\
\text { Local Search }\end{array}$ \\
\hline (CHANG; LEE, 2018) & $\checkmark$ & TSP & NL & - \\
\hline (PONZA, 2016) & $\checkmark$ & TSP & - & $\begin{array}{l}\text { Simulated } \\
\text { Annealing }\end{array}$ \\
\hline (MATHEW; SMITH; WASLANDER, 2015) & $\checkmark$ & TSP & OPP & - \\
\hline (OTHMAN et al., 2017) & $\checkmark$ & TSP & - & - \\
\hline (ULMER; THOMAS, 2018) & $\checkmark$ & VRP & - & $\begin{array}{l}\text { Policy Function } \\
\text { Approximation }\end{array}$ \\
\hline This dissertation & $\checkmark$ & VRP & MILP & $\begin{array}{l}\text { Hybrid Genetic } \\
\text { Algorithm }\end{array}$ \\
\hline
\end{tabular}

Source - Authors. 

CHAPTER



PROBLEM DEFINITION AND MODEL

In this chapter the problem and how it is going to be approached in this dissertation is discussed. Firstly, two scenarios are presented to exemplify how the use of drone is advantageous, and why such research is relevant. Afterwards, we present the Mixed-Integer Linear Programming (MILP) formulation to the problem.

\subsection{Scenario I}

Large cities' downtown areas are heavily crowded with traffic and people, which make a delivery truck's access difficult or even prohibited (outside work hours, usually). An alternative to solve, or at least minimize these problems could be delivering packages with drones. The truck would navigate around these traffic heavy regions, and drones could fly into downtown and deliver packages to roofs of buildings or special sites set-up for this task, avoiding traffic, and speeding up delivery time. Additionally, if their use reduced the number of vehicles in central areas, pollution would decrease and air quality would improve.

Figure 4 shows an example of this case, where the trucks are surrounding the city center, serving clients that are not eligible for drone delivery (red and orange marks), and the drones start flying towards delivery points (green and blue marks). It is worth noting that all drone-eligible clients are also truck-eligible, since all the shipment is within the truck's cargo. Since a drone's weight capacity is limited, possible applications could be mail, documents, food delivery and other small packages with low weight.

\subsection{Scenario II}

Rural areas and small cities tend to have large distances between one delivery point to another, or some access roads are not paved (specially in underdeveloped countries). 
Figure 4 - First scenario of drone usage in logistics: delivery in downtown areas.

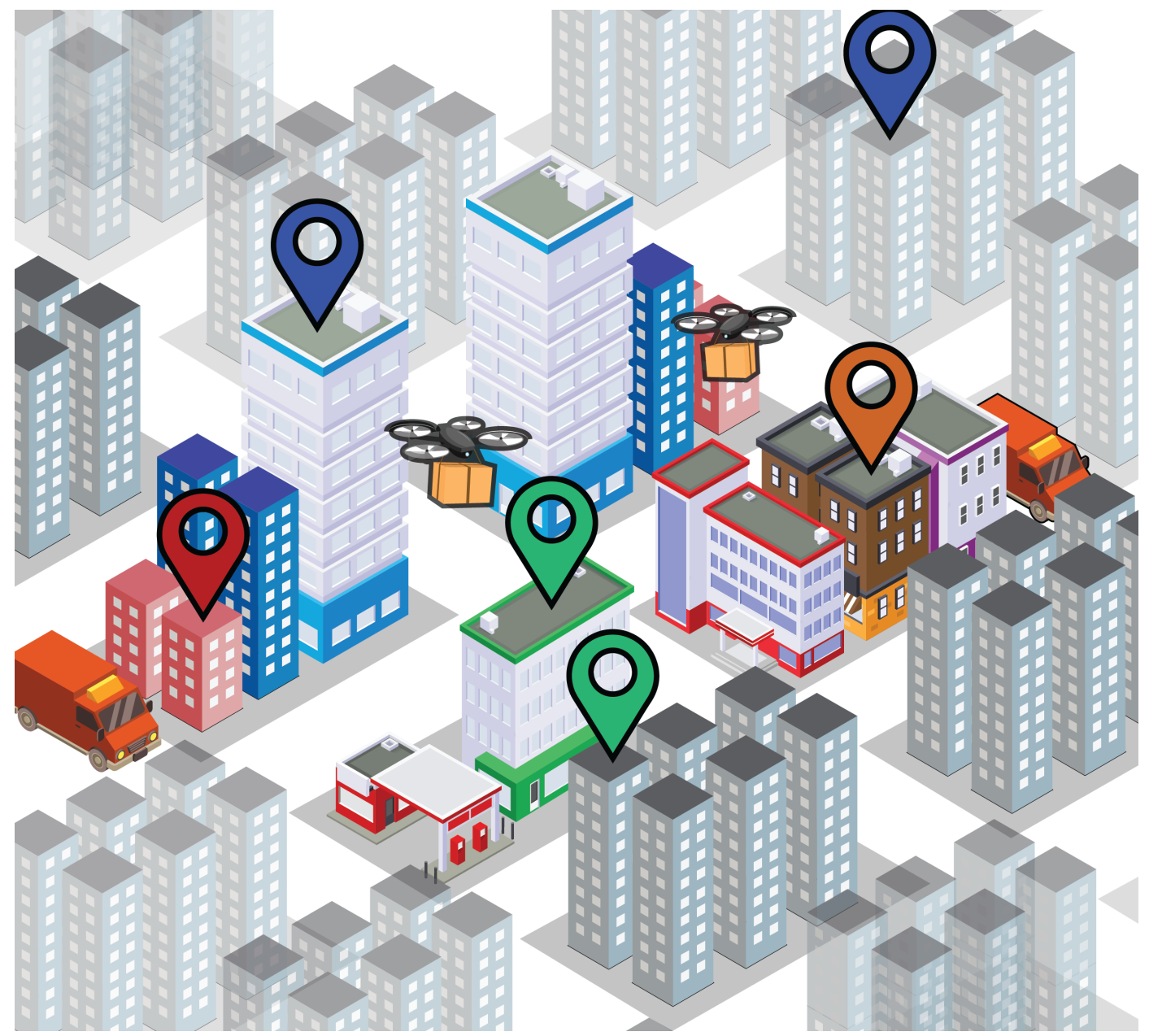

Source: Authors, Based on Designed by Freepik

Drone usage in this case could help to heavily reduce the truck's mileage and avoid riskier roads. A truck attending one client can send the drone to another one that stands outside the main road, and meet the truck back at the next client's location. Rural areas also have more space where a drone can safely land.

Figure 5 shows an example of rural area where a drone can save time, and the trucks can keep delivering along the main road, while the drones deliver to the side roads. Also in this case, aside from any road condition, some packages will be delivered by truck, since not all packages are drone-eligible.

\subsection{Problem Discussion}

Given the above scenarios, the first one is somewhat complicated to turn into reality in today's world, since several problems are present: privacy, for one, since modern drones use cameras to process their flight path; the risk of failure, where the drone can fall 
Figure 5 - Second scenario of drone usage in logistics: delivery in rural areas

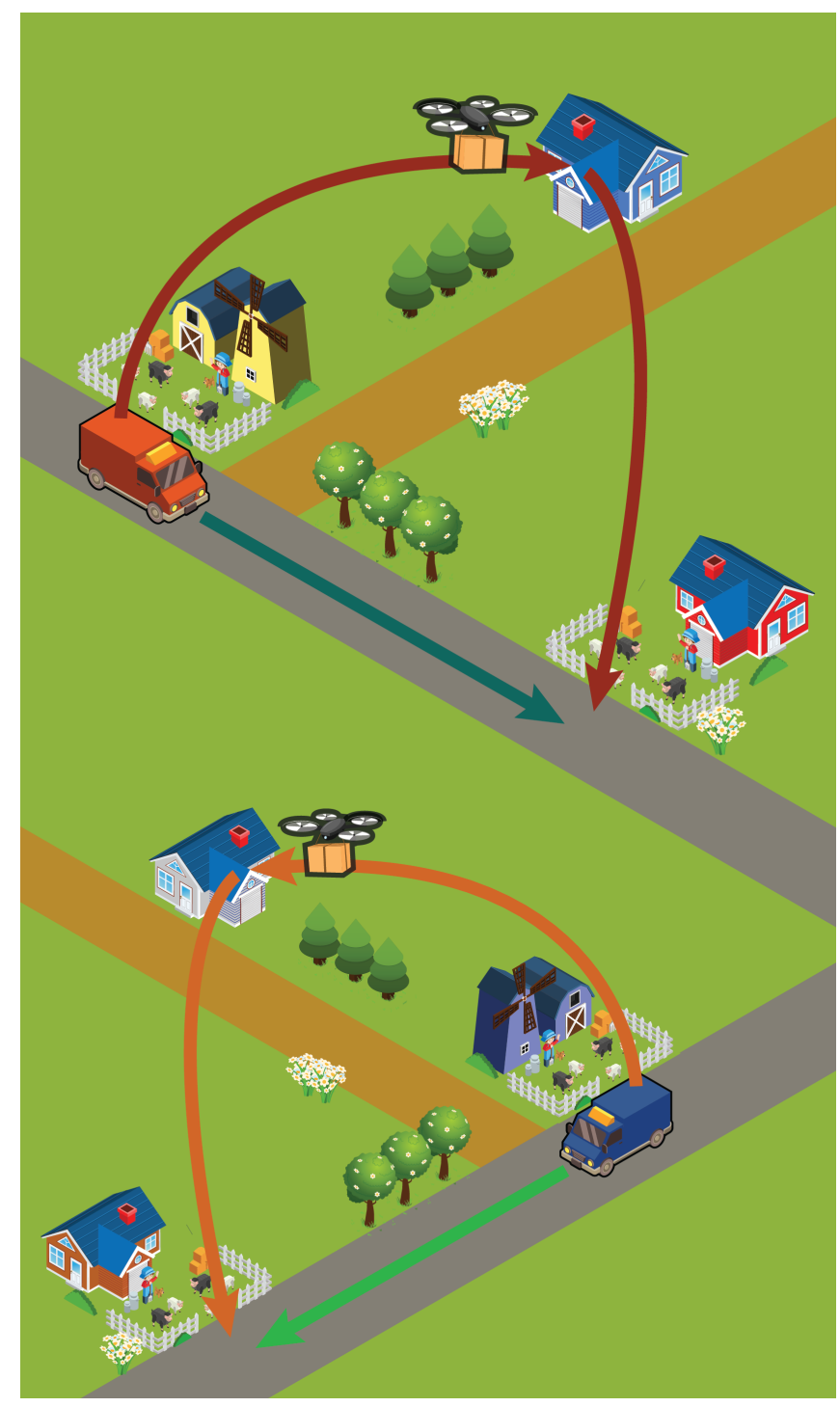

Source: Authors, Based on Designed by Freepik

to the ground and hurt a pedestrian or damage a property; air space shared with other aircrafts (planes, helicopters) increasing the risk of accidents.

The second scenario is more likely to happen within a few years, since most of the problems from the first one are a lot less probable. Large areas with no buildings or people, and the large distances allow drones to be used to their full potential, though security should not be disregarded. This scenario would save a lot of time for the truck, allowing more packages to be delivered within the same number of hours or to have reduced mileage (resulting in fewer emissions of greenhouse gases). Both of these scenarios were reported by DHL to be the most promising for logistic companies in the next years (KüCKELHAUS; NIEZGODA; ENDRIß, 2014). Despite the complications, the advantages are overwhelming, reducing costs, time and pollution. 
However, these scenarios will only be efficient after answering the following question: How to decide which clients are served by the truck and which are served by the drone in an optimal way? The answer to this question could be achieved by solving a VRP, where the routes contain which clients are to be served by which vehicle. Since the number of customers is expected to be very large (from logistics companies' standpoint), a TSP solution would not suffice, therefore a VRP approach needs to be proposed. However, it is not an easy task to achieve, because the problem has several particularities that need to be taken into account. Hence, before extending the model, some assumptions and considerations are required:

- Each fleet type is homogeneous, all the drones have the same capacity $Q^{v}$, endurance $e$ and speed $v_{d}$; similarly, all the trucks have the same capacity $C_{t}$ and speed $v_{t}$, the trucks' endurance is ignored due to it being sufficiently large.

- The drone can only take-off and land while the truck is at a client location or the depot, no extra stops are allowed.

- A drone is linked to only one truck, meaning the drones cannot depart from one truck and arrive at another.

- If a drone has to wait for its truck, the drone cannot land so as to preserve energy, and will have to hover.

- Not all clients can be served by a drone, meaning some packages are required to be delivered by truck.

- Each drone can only carry one package, even if its capacity allows it for more, meaning it needs to return to its truck before it makes another delivery.

- No drone nor truck can visit the same client more than once.

- All customers have time-windows and service time pre-established. However, the service time is only taken into account for the truck deliveries. We assume that the drones will just deliver the product and leave, while the deliveryman has to do extra work other than deliver, such as unload the truck, get the customer's signature, or some other time consuming task.

- The time to make the package flight-ready is incorporated in the prep time for the drone launch. Additionally, any time related to a returning drone is incorporated to the landing time.

- The depot has a landing site which support the number of available drones.

Based on these considerations we present in the next section the MILP for the VRPD. 


\subsection{The VRPD Mixed-Integer Linear Programming Model}

The mathematical programming model for the Vehicle Routing Problem with Drones is presented. In this model, based on (MURRAY; CHU, 2015), some adjustments were made to either make the model less computationally expensive, or to encourage the drone usage, for example, by relaxing the requirement that a drone needs to arrive at the final depot together with its truck.

The MILP model has several sets, parameters and variables that are explained next. All constraints are grouped up by their goal with a briefly description, and are presented afterwards.

Set:

$C: \quad$ set of clients;

$C^{\prime}: \quad$ set of clients drone-eligible;

$N$ : $\quad$ set of nodes with starting point 0 and ending point $c+1$ being the depot;

$N_{0}$ : $\quad$ set of nodes which vehicles may depart from, i.e. $0,1, \ldots, c$;

$N_{+}$: $\quad$ set of nodes which vehicles may arrive at, i.e. $1,2, \ldots, c+1$;

$P: \quad$ set of nodes which combined can form a drone route, i.e $(i, j, k)$ such that $i \in N_{0}, j \in C^{\prime}, k \in N_{+}$;

$M$ : $\quad$ set of vehicles that can be used.

Data:

$d_{i j}: \quad$ the distance between vertices $i$ and $j$, for trucks;

$d_{i j}^{\prime}$ : the distance between vertices $i$ and $j$, for drones;

$q_{i}$ : $\quad$ the demand of client $i$;

$Q^{t}: \quad$ the truck's load capacity;

$a_{i}: \quad$ the start of the time windows for client $i$;

$b_{i}$ : $\quad$ the end of the time windows for client $i$;

$s_{i}$ : the service's duration for client $i$ (note: 0 for drone deliveries);

$v_{d}$ : the drones' average flight speed;

$v_{t}$ : the trucks' average speed;

$e: \quad$ the drones' flight endurance;

$S_{L}$ : $\quad$ set-up time for launching a drone from one truck;

$S_{R}$ : $\quad$ set-up time for returning a drone from one truck;

$T_{\text {max }}$ : maximum operational time (closing window of depot);

$K$ : a large enough number. 


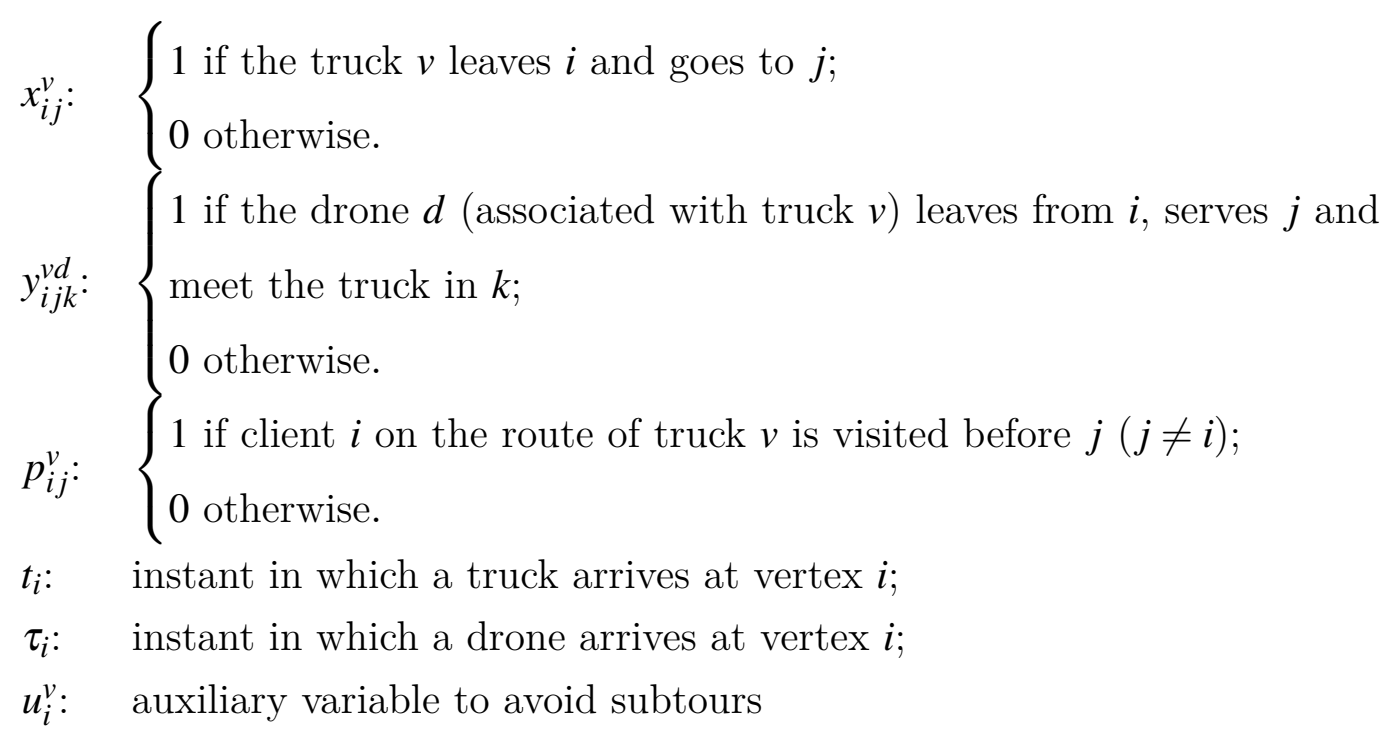

The objective function is the classical VRP objective, which aims to reduce the total distance, and will be used to compare this model with the classical known results.

Objective Function I (Equation 3.1): to minimize the total distance traversed by the trucks (i.e., maximizing the distance traveled by the drones).

$$
\operatorname{Min} Z=\sum_{i \in N_{0}} \sum_{\substack{j \in N_{+} \\ i \neq j}} \sum_{\nu \in M} d_{i j} x_{i j}^{v}
$$

Constraints 3.2 ensure every customer $j$ is visited by either a truck or a drone.

$$
\text { s.t. } \sum_{\substack{i \in N_{0} \\ i \neq j}} \sum_{v \in M} x_{i j}^{v}+\sum_{\substack{i \in N_{0} \\ i \neq j}} \sum_{\substack{k \in N_{+} \\(i, j, k) \in P}} \sum_{(v, d) \in M} y_{i j k}^{v d}=1 \quad \forall j \in C
$$

Constraints 3.3 and 3.4 ensure that every truck leaves the depot and returns to the depot. Here the trucks are allowed to leave from the starting depot to the ending depot because some customers could be served by drones directly from the depot.

$$
\begin{array}{cl}
\sum_{j \in N_{+}} x_{0 j}^{v}=1 & \forall v \in M \\
\sum_{i \in N_{0}} x_{i(c+1)}^{v}=1 & \forall v \in M
\end{array}
$$

Constraints 3.5 prevents sub-cycles for each vehicle $v$.

$$
u_{i}^{v}-u_{j}^{v}+1 \leq(c+2)\left(1-x_{i j}^{v}\right) \quad \forall i \in C, j \in\left\{N_{+}: j \neq i\right\}, v \in M
$$

The flow constraints 3.6 ensures that a truck $v$ will arrive and leave the customer $j$.

$$
\sum_{\substack{i \in N_{0} \\ i \neq j}} x_{i j}^{v}=\sum_{\substack{k \in N_{+} \\ k \neq j}} x_{j k}^{v} \quad \forall j \in C, v \in M
$$


Constraints 3.7 and 3.8 limits a drone $d$ to arrive and leave a customer at most once.

$$
\begin{aligned}
& \sum_{\substack{j \in C^{\prime} \\
j \neq i}} \sum_{\substack{k \in N_{+} \\
(i, j, k) \in P}} y_{i j k}^{v d} \leq 1 \quad \forall i \in N_{0},(v, d) \in M \\
& \sum_{\substack{i \in N_{0} \\
i \neq k}} \sum_{\substack{j \in C^{\prime} \\
(i, j, k) \in P}} y_{i j k}^{v d} \leq 1 \quad \forall k \in N_{+},(v, d) \in M
\end{aligned}
$$

Constraints 3.9 ensure that the truck $v$ will visit customer $i$ and $k$ if the correspondent drone $d$ does the route $i \rightarrow j \rightarrow k$. Constraints 3.10 do the same when $i=0$ (so the truck only needs to visit $k$ ).

$$
\begin{gathered}
2 y_{i j k}^{v d} \leq \sum_{\substack{h \in N_{0} \\
h \neq i}} x_{h i}^{v}+\sum_{\substack{l \in C \\
l \neq k}} x_{l k}^{v} \\
\qquad i \in C, j \in\{C: j \neq i\}, k \in\left\{N_{+}:(i, j, k) \in P\right\},(v, d) \in M \\
y_{0 j k}^{v d} \leq \sum_{\substack{h \in N_{0} \\
h \neq k}} x_{h k}^{v} \quad \forall j \in C, k \in\left\{N_{+}:(0, j, k) \in P\right\},(v, d) \in M
\end{gathered}
$$

Constraints 3.11 ensure that the truck $v$ will have $i$ before $k$ in its route, if the correspondent drone $d$ does the route $i \rightarrow j \rightarrow k$.

$$
u_{k}^{v}-u_{i}^{v} \geq 1-(c+2)\left(1-\sum_{\substack{j \in C^{\prime} \\(i, j, k) \in P}} y_{i j k}^{v d}\right) \quad \forall i \in C, k \in\left\{N_{+}: k \neq i\right\},(v, d) \in M
$$

The next set of constraints ensure that a truck and its drone will have a synchronous time in both take-off and landing customers. The first two (3.12 and 3.13) synchronize the arrival at customer $i$, whilst the next two 3.14 and 3.15 synchronize the arrival at customer $k$. Here, the "big M" $K$ equals to $T_{\max }$, since the biggest difference (for non bounding cases) in any time schedule for the VRPTW is less than the maximum operational time.

$$
\begin{aligned}
& \tau_{i} \geq t_{i}-K\left(1-\sum_{\substack{(v, d) \in M \\
j}} \sum_{\substack{j \in C \\
j \neq i}} y_{i j \in N_{+}}^{v d}\right) \quad \forall i \in C
\end{aligned}
$$

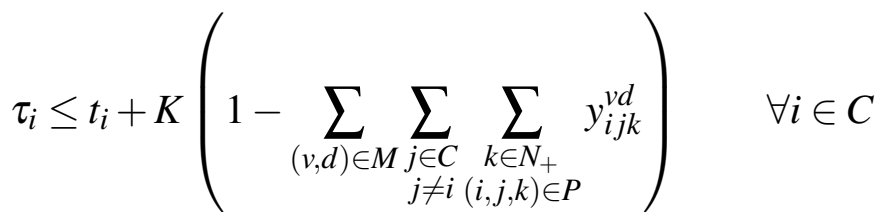




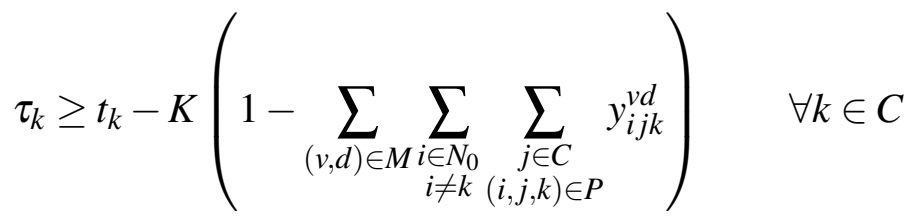

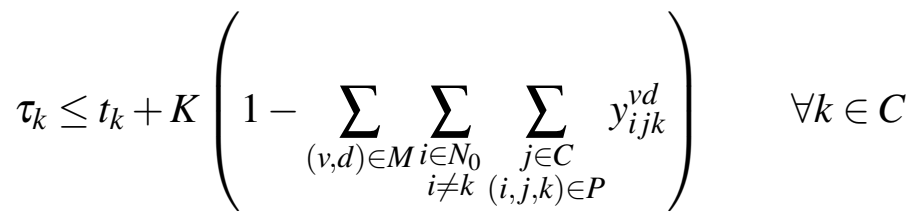

$$
\begin{aligned}
& K=T_{\max }
\end{aligned}
$$

Constraints 3.16 and 3.17 are related to the truck $v$ arrival time at customer $k$, and the depot $c+1$, respectively. They account the travel time from $h$ to $k($ or $c+1)$, the service time at customer $h$ (in case $h$ is the depot, the service time is 0 ) and possible takeoff and landing times ( $S_{L}$ and $S_{R}$, respectively) from the drone $d$. Here $K$ also incorporates the travel time, service time and take-off (for the first case) and landing times.

$$
\begin{aligned}
& t_{k} \geq t_{h}+\frac{d_{h k}}{v_{t}}+s_{h}+S_{L}\left(\sum_{\substack{l \in C \\
l \neq k}} \sum_{\substack{m \in N_{+} \\
(k, l, m) \in P}} y_{k l m}^{v d}\right)+S_{R}\left(\sum_{\substack{i \in N_{0} \\
i \neq k}} \sum_{\substack{j \in C \\
i, j, k) \in P}} y_{i j k}^{v d}\right)-K\left(1-x_{h k}^{v}\right) \\
& \forall h \in N_{0}, k \in\{C: k \neq h\},(v, d) \in M \\
& K=T_{\max }+\frac{d_{h k}}{v_{t}}+S_{L}+S_{R}+s_{h} \\
& t_{(c+1)} \geq t_{h}+\frac{d_{h(c+1)}}{v_{t}}+s_{h}++S_{R}\left(\sum_{i \in N_{0}} \sum_{\substack{j \in C \\
(i, j, c+1) \in P}} y_{i j(c+1)}^{v d}\right)-K\left(1-x_{h(c+1)}^{v}\right) \\
& \forall h \in N_{0},(v, d) \in M \\
& K=T_{\max }+\frac{d_{h(c+1)}}{v_{t}}+S_{R}+s_{h}
\end{aligned}
$$

Constraints 3.18 ensure that the time of visit for customer $j$, served by the drone $d$, accounts the travel time from $i$ to $j$ and the time the drone left $i$. This constraint is bounding when the drone does travel from $i$ to $j$ and then to some $k$, otherwise, the "big M" $K$ is equal to $T_{\max }$ plus the travel time from $i$ to $j$.

$$
\tau_{j} \geq \tau_{i}+\frac{d_{i j}^{\prime}}{v_{d}}-K\left(1-\sum_{\substack{k \in N_{+} \\(i, j, k) \in P}} y_{i j k}^{v d}\right)
$$

$$
\forall j \in C^{\prime}, i \in\left\{N_{0}: i \neq j\right\},(v, d) \in M
$$




$$
K=T_{\text {max }}+\frac{d_{i j}^{\prime}}{v_{d}}
$$

Constraints 3.19 and 3.20 are similar to the above, but for the customer $k$ or depot $c+1$, respectively, if the drone lands there $(i \rightarrow j \rightarrow k)$. The main difference is the landing time $S_{R}$ that needs to be added, as well as for $K$.

$$
\begin{gathered}
\tau_{k} \geq \tau_{j}+\frac{d_{j k}^{\prime}}{v_{d}}+S_{R}-K\left(1-\sum_{\substack{i \in N_{0} \\
(i, j, k) \in P}} y_{i j k}^{v d}\right) \\
K=T_{\text {max }}+\frac{d_{j k}^{\prime}}{v_{d}}+S_{R} \\
\tau_{(c+1)} \geq \tau_{j}+\frac{d_{j(c+1)}^{\prime}}{v_{d}}+S_{R}-K\left(\begin{array}{c}
1-\sum_{\substack{i \in N_{0} \\
(i, j, c+1) \in P}} y_{i j(c+1)}^{v d} \\
K=T_{\text {max }}+\frac{d_{j(c+1)}^{\prime}}{v_{d}}+S_{R}
\end{array} \quad \forall j \in C^{\prime},(v, d) \in M\right.
\end{gathered}
$$

Constraints 3.21 ensure that the drone endurance $e$ is not exceeded. This endurance is not cumulative, which means that the batteries are either fully recharged or swapped after each trip. Here the "big M" cannot be larger than the longest possible waiting time reduced by the endurance.

$$
\begin{gathered}
\tau_{k}-\tau_{i} \leq e+K\left(1-\sum_{\substack{j \in C \\
(i, j, k) \in P}} y_{i j k}^{v d}\right) \\
K=T_{\max }-e \\
\quad \forall k \in C, i \in C,(v, d) \in M
\end{gathered}
$$

Constraints 3.22, 3.23 and 3.24 set the variables $u_{i}$ and $p_{i j}$ for each truck $v$.

$$
\begin{gathered}
u_{i}^{v}-u_{j}^{v} \geq 1-(c+2) p_{i j}^{v} \quad \forall i \in C, j \in\{C: j \neq i\}, v \in M \\
u_{i}^{v}-u_{j}^{v} \leq-1+(c+2)\left(1-p_{i j}^{v}\right) \quad \forall i \in C, j \in\{C: j \neq i\}, v \in M \\
p_{i j}^{v}+p_{j i}^{v} \leq 1 \quad \forall i \in C, j \in\{C: j \neq i\}, v \in M
\end{gathered}
$$


Constraints 3.25 cover the drone not leaving from another customer while it is still in the middle of a delivery. Figure 6 shows the unfeasible cases that are avoided by these constraints.

$$
\begin{aligned}
& \tau_{l} \geq \tau_{k}-K\left(3-\sum_{\substack{j \in C \\
(i, j, k) \in P \\
j \neq l}} y_{i j k}^{v d}-\sum_{\substack{m \in C \\
m \neq i \\
m \neq k \\
m \neq k \\
m \neq l \\
m \neq n}} \sum_{\substack{n \in N_{+} \\
n \neq i \\
n \neq k}} y_{l m n}^{v d}-p_{i l}^{v}\right) \\
& \forall i \in N_{0}, k \in\left\{N_{+}: k \neq i\right\}, l \in\{C: l \neq i, l \neq k\},(v, d) \in M \\
& K=T_{\max }
\end{aligned}
$$

Constraints 3.26 ensure the vehicles load capacities are respected.

$$
\sum_{i \in N_{0}} q_{i} \sum_{\substack{j \in C \\ j \neq i}} x_{i j}^{v}+\sum_{j \in C} q_{j} \sum_{\substack{i \in N_{0} \\ i \neq j}} \sum_{\substack{\left.k \in N_{+} \\ i, j, k\right) \in P}} y_{i j k}^{v d} \leq Q^{v} \quad \forall v \in M
$$

Constraints 3.27 and 3.28 are symmetry breaking constraints. The first one ensures the trucks are used in order, while the second one assures the trucks visit order is ascending, i.e. a truck $v(v>1)$ can only start its route by visiting a customer which has greater index than the truck's $v-1$ first customer.

$$
\begin{gathered}
x_{0 j}^{v} \leq x_{0 j}^{v-1} \quad \forall j \in C, v \in M-\{0\} \\
x_{0 j}^{v} \leq \sum_{\substack{l \in C \\
l<j}} x_{0 l}^{v-1} \quad \forall j \in C, v \in M-\{0\}
\end{gathered}
$$

The next constraints determines the start value and the domains of the variables.

$$
\begin{gathered}
t_{0}=0 \\
\tau_{0}=0 \\
p_{0 j}^{v}=1 \quad \forall j \in C, v \in M \\
x_{i j}^{v} \in\{0,1\} \quad \forall i \in N_{0}, j \in\left\{N_{+}: j \neq i\right\}, v \in M
\end{gathered}
$$




$$
y_{i j k}^{v d} \in\{0,1\}
$$

$$
\begin{gathered}
\forall i \in N_{0}, j \in\{C: j \neq i\}, k \in\left\{N_{+}:(i, j, k) \in P\right\},(v, d) \in M \\
1 \leq u_{i}^{v} \leq c+2 \quad \forall i \in N_{+}, v \in M \\
a_{i} \leq t_{i} \leq b_{i} \quad \forall i \in N \\
a_{i} \leq \tau_{i} \leq b_{i} \quad \forall i \in N \\
p_{i j}^{v} \in\{0,1\} \quad \forall i \in N_{0}, j \in\{C: j \neq i\}, v \in M
\end{gathered}
$$

\subsection{Additional Notes on the Model}

The MILP developed has some characteristics that require further notes. For instance, the addition of classical symmetry breaking constraints (3.27 and 3.28) are possible only for the trucks' routes, since they are the only independent source. And the possible drones symmetry problems are very narrow, the main case is when there are different drones serving customers directly from the depot and returning.

The variables $p_{i j}^{v}$ and $u_{i}^{v}$, unlike the classical models from literature, are added the vehicle index because the order of visit for each customer is relevant for the drone $d$ linked with the truck $v$. The constraints 3.25 use the variable $p$ to check whether the truck $v$ visits $i$ after $l$. The original version of this constraint works because in the FSTSP all customers are visited by the same vehicle (if not visited by drone, obviously), therefore, the variable always makes sense. However, in the VRPD, customer $i$ and $l$ may be from different routes and, thus, losing its original intent. By adding the vehicle index, the value of $p$ is now guaranteed to have both customers $i$ and $l$ in the same route, if the case is so. This restriction is very important to the problem since it avoid the possibility of mixed drone routes, where the single drone would be launched more than once before returning to the truck (as shown in Figure 6). This change implicates in the constraint 3.24 being less than equal, rather than equal to 1 , since $i$ is not necessarily before $j$ (or vice-versa).

Unlike the original model, a decision was make to allow the drone to return to the depot by itself, as seen in constraints 3.14 and 3.15 where the synchronous return point $k$ contains only the customer set $C$. Considering that a drone which is not going to delivery to any other customer, then it should be okay for it to return to the depot. This way the 
Figure 6 - Avoided scenarios

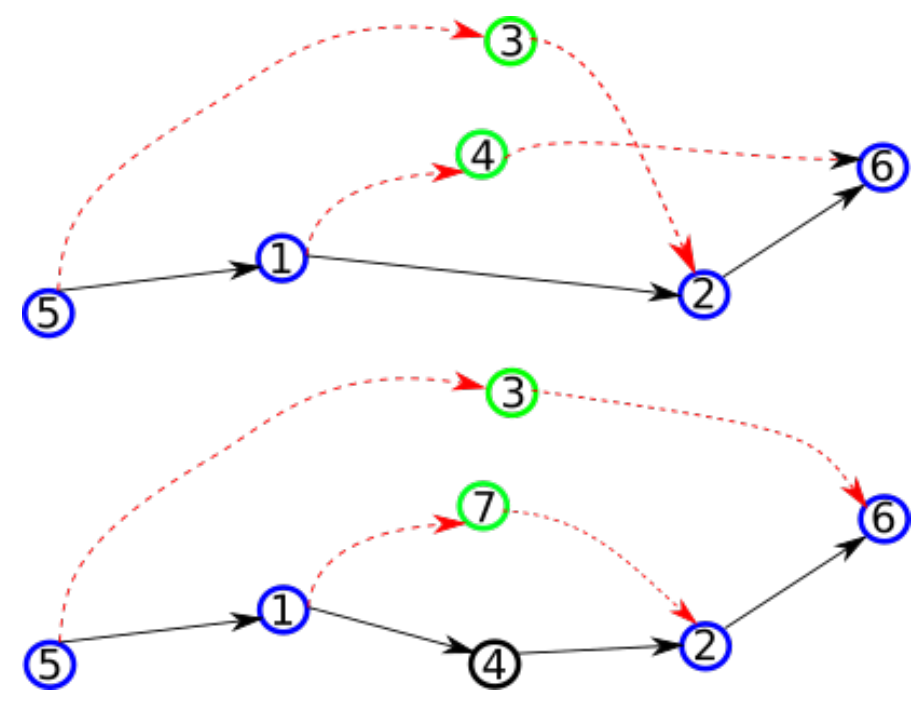

Source: Authors

drone could have its battery charged for the next operation and its basic maintenance taken care as soon as possible, whilst also allowing for more drone routes, as both the trucks and drones will not depend on the next customers' time-windows to synchronize arrivals.

Similarly to the original model, the objective function can be changed to minimize the time of return to the depot. Objective Function $I I$ : to minimize the time of return to the depot, hence minimizing all delivery times. This is the function used by (MURRAY; CHU, 2015).

$$
\operatorname{Min} t_{(c+1)}
$$

In the next chapter (4), we present the HGA approach. 
CHAPTER

4

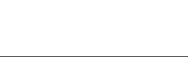

\section{HYBRID GENETIC ALGORITHM}

This chapter focuses on the Hybrid Genetic Algorithm (HGA) developed for the Vehicle Routing Problem with Drones (VRPD). Our HGA utilizes a combination of a Genetic Algorithm (GA) and a Local Search (LS). The GA is an evolutionary algorithm that start with several solutions which, as the name says, evolve into a better fit population; while the LS is a method in which one solution has one or more of its neighborhoods explored to find better results. The combination of GA and LS was first successfully used in solving a VRP by (PRINS, 2004). The version of the HGA done in this dissertation, however, is more similar and was inspired by the work of (VIDAL et al., 2014), although they share similarities.

The HGA used follows the general guideline of a traditional GA: a initial population is created (each individual is represented by a chromosome); a selection within the population where two parents are chosen occurs; these parents are combined in a crossover operation; and finally the children may be altered in some way as part of the mutation process and are returned to the population. However, as in (VIDAL et al., 2014), instead of a possible mutation step there is an education step. In the education step, the LS procedures are applied to the offspring in order to improve the next generation in a more efficient way. The education also contains the drone route decision method, a heuristic to find a good route for the drone.

\subsection{HGA}

Algorithm 1 describes the structure of the HGA. The parameters for the algorithm are the size of the population $n$, the maximum number of iterations (number of generations and/or execution time) and the maximum size of the population, as it is of variable size. The algorithm start by generating the initial population, which is then evaluated (lines 3 and 4). The evaluation step temporarily stores in the memory the fitness of all the 
chromosomes in a way that the selection process does not need to compute it again. Using that temporary memory, the selection process occurs (line 7) and two distinct parents are selected to perform the Crossover process (line 8). The crossover will return at most two offspring (already evaluated in the process), which will be passed to the Education step (line 9). The children then are added to the population. If the maximum size of the population is reached, the population is trimmed (line 12), i.e. the least fit individuals are removed from the population. The best individual is updated if needed in the final step (line 15). The procedure does this while the time limit or the maximum number of generations is not reached, returning the best individual. The main steps of the HGA are further elaborated in the next sections.



The chromosome structure, which is a mix of the traditional single route without trip delimiters used in TSP problems combined with a second array with the drone information, is explained in the next section.

\subsection{Chromosome Representation}

One of the main concerns of any computational procedure is representing a real world solution computationally without loss of information. Solutions from mathematical models are very similar to real solutions. However, this is not the case for other types of solutions. For instance, in metaheuristics, usually there are generic procedures that 
can be applied to several types of problems. These generic procedures, therefore, need to be adapted to the problem being solved. And with adaptation, usually comes a loss of information or precision in the data. The goal would be to minimize this loss of information without making it too difficult to use the metaheuristic's original generic format. This struggle was present when trying to represent VRPD solutions.

Traditional GA representations for VRP solutions such as binary values or routes with delimiters fails to account for drone deliveries. However, as in the work of (VIDAL et al. , 2012), the traditional schemes utilized could be altered in a way that fits the problem's particularities.

The chromosome for the VRPD's HGA is represented with a two by $n$ matrix $(2 \times n$, $n$ being the number of customers) where: the first row is a single route without delimiters, containing each customer in the order they should be visited; and the second row is a binary vector to determine if a customer is served by truck or drone. The corresponding customer from the first row will have the value 1 if it is served by the drone and 0 otherwise (as seen in Figure 7).

The single route without delimiters, although initially used for the TSP, can be used for VRP variants because it can be split into different routes. For example, in (PRINS, 2004) the author utilizes an algorithm of order $O\left(n^{2}\right)$ to split the routes by building an auxiliary graph and returning minimum cost paths. Based on that approach we built one split algorithm for the VRPD which is detailed next.

Figure 7 - Chromosome representation.

6432517 Route

01010001 Drone status

Source: Authors.

\subsubsection{Split Procedure}

We illustrate this split with Figure 8, where each route shows it own section of a single route chromosome. The customers from where drones launch or land are colored blue (just for customers' locations, the depot in the example is also a launch and landing point) and are served by the truck. The green circles represent the customers served by the drones. Black circles are supplied by the truck. The routes although disconnected here, can be joined with any permutation of those routes. This can lead to different chromosomes having the same fitness value, as they are the same routes, only in different order. The Split procedure recovers the trips from the single route chromosome by utilizing some characteristics from the problem's instance to improve computational performance. Firstly, we build a graph $G$ given the problem input, the customers and their time-windows. 
Figure 8 - Chromosome translation and split example.

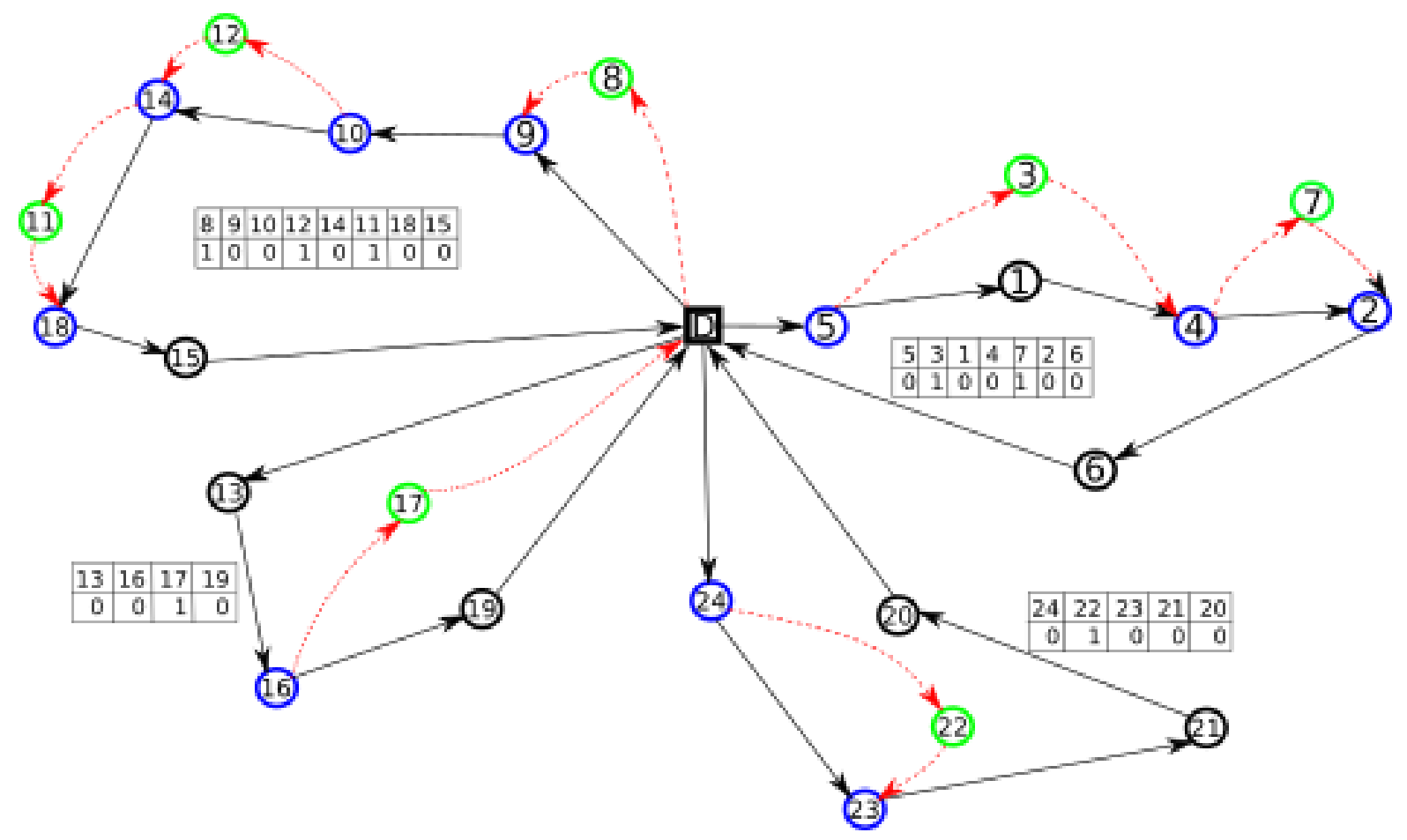

Source: Authors.

$G$ contains each customer as nodes, where the edges between them are present if, and only if, it is possible for going from one customer to another. For example, the time windows of two customers may be overlapping, and it might be impossible to visit them in sequence because of that. To calculate whether it is possible, we use the following inequation:

$$
a_{i}+\text { travel_time }(i, j)+S_{i} \leq b_{j}
$$

If the Inequation 4.1 is false, then it is not possible to visit $j$ after visiting customer $i$, because even if arriving at the earliest possible at $i$, the shortest time possible to arrive at $j$ would be after its time window closes.

The graph $G$ is built with every customer pair using the Inequation 4.1. This graph is independent of current solutions, therefore it is calculated before the start of the HGA. The graph $G$ is the input, along the chromosome for the Split procedure, as seen in Algorithm 2. The algorithm consists of forming new routes as long as the next customer in the chromosome does not break the load capacity or the time window (line 11), including waiting for the window to open if necessary. When checking the customer or when storing the customer's identifier (lines $8,10,13,14,24$ ), we refer to the first row of the chromosome matrix. However, both rows will be saved in the other cases. This algorithm has $O(n)$ complexity. 


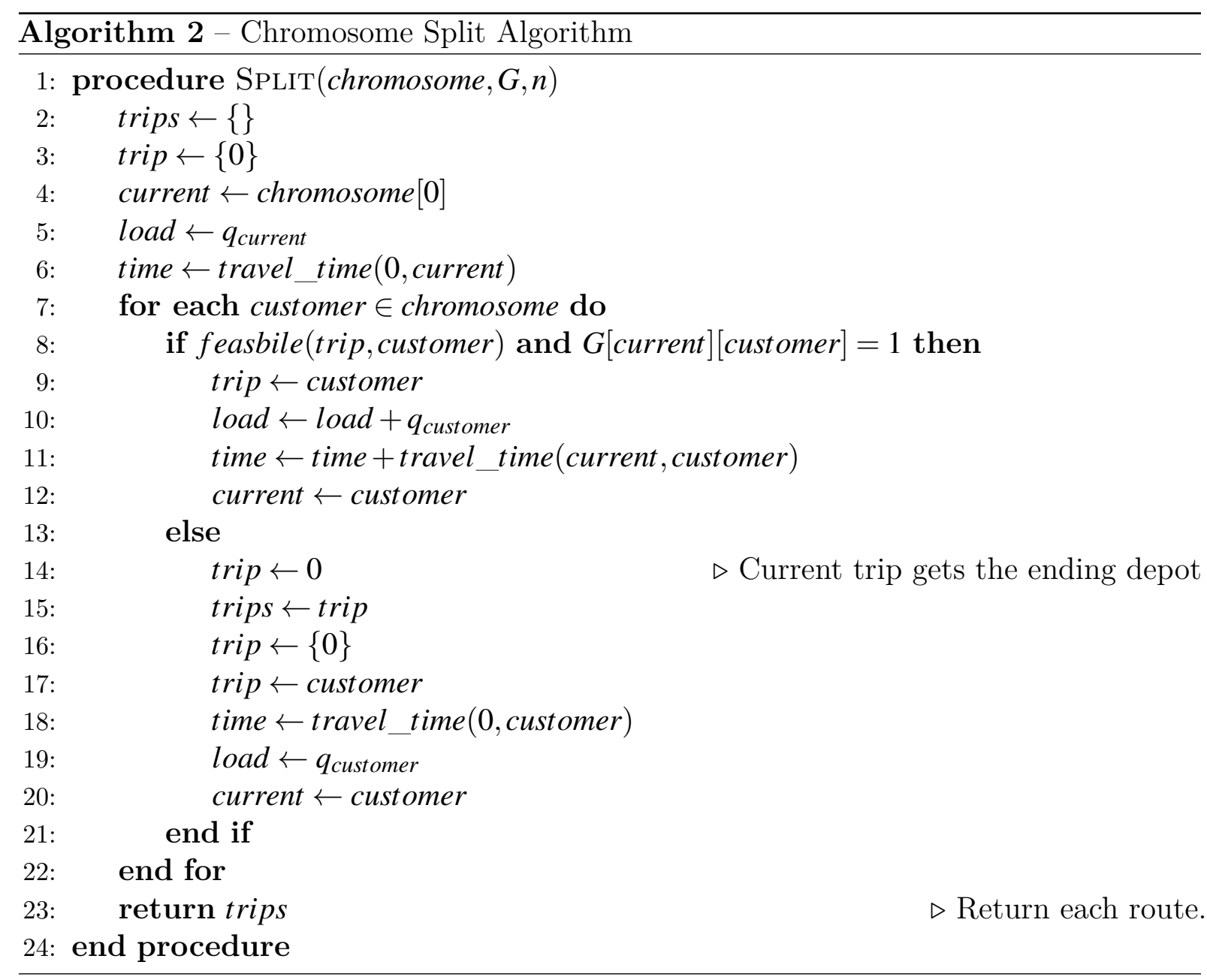

This algorithm is greedy, thus it does not optimally split the chromosome. For example, in a case where the route of a drone consists in visiting a customer directly from the depot and back, the split is unlikely to return that as a solution. Therefore the HGA utilizes another auxiliary structure which saves the routes separately. This will happen when the Local Search finds better routes, and will be discussed in the Education section. Next, we specify how the initial population is created.

\subsection{Initial Population}

The efficiency of most heuristics is heavily dependent on the initial solution (or solutions, for population based heuristics). Since the algorithms explore the characteristics of current solutions, the better they are, the better the performance overall. To better exploit this concept, we created one initial population based on the sweep heuristic, completed with random solutions to add more variability.

We start the population by adding chromosomes generated by an adapted sweep heuristic, as seen in Algorithm 3. The sweep heuristic was proposed by (WREN; HOLLIDAY , 1972) and it works by adding customers based on its angle when compared to the 
depot. This aims to put customers that are around the same region in the same route, since customer with similar angles are supposedly closer to each other. It is a very simple heuristics that can return one route with less computational effort. The sweep used here does not take the drones into account, so it builds truck-only routes for the starting population. Since we want several routes, we change the starting angle for each route, avoiding duplicates.



Since we want to diversify our population, some customers are generated by the 
random algorithm 4. It simply returns non-duplicates of random permutation of customers. The difference here is that we allow drones to serve customers. The parameter drone_served is the probability in which each drone-eligible customer has of being served by a drone. Given this initial population, the next step consists of selecting the individuals to perform a crossover.

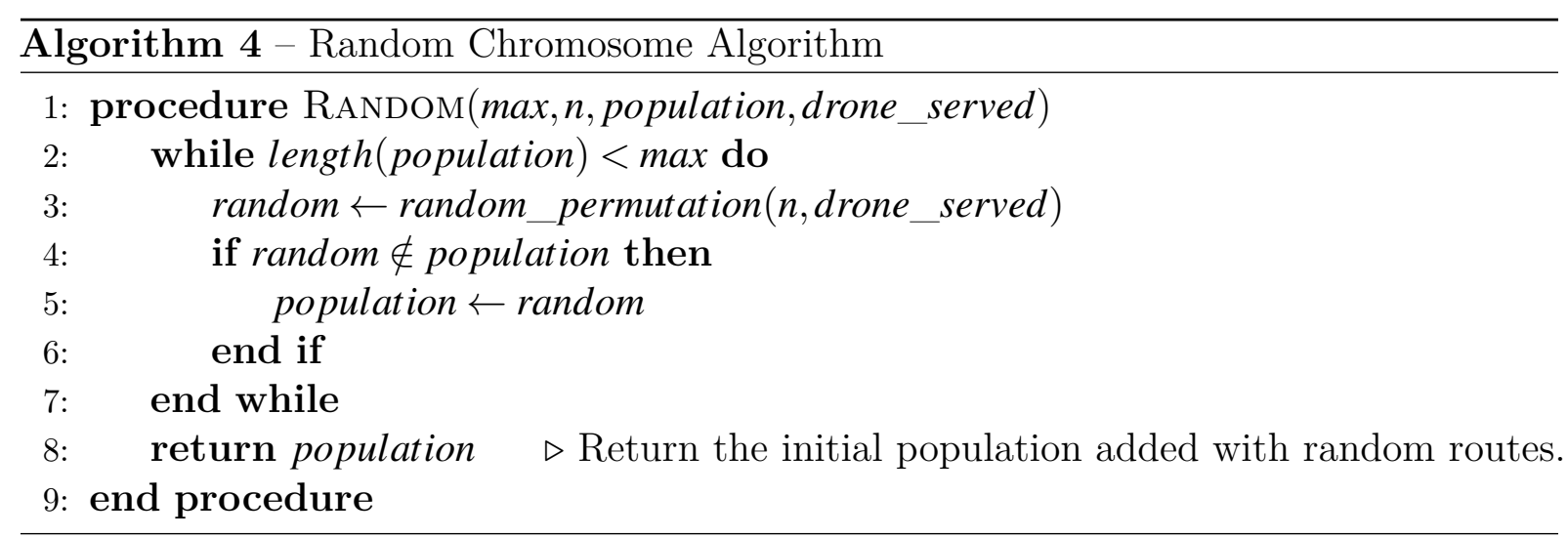

\subsection{Crossover and Selector Operators}

The crossover step is very important for the performance of a GA. If the crossover function is too simple, the population might take longer to evolve. And if it is too complex, it might take too much time to compute or could make the population converge too quickly. However, it is maybe even more crucial to the growth (fitness-wise) of the population to select which parents will breed. Therefore, before presenting the crossover method, we introduce the selection process.

There are several selection methods utilized in GA literature: Tournament, roulette wheel, best fit, to name a few. The selection method we chose is the traditional tournament without replicates. This method selects $n$ individuals from the population at random, and the best two of these are selected to become the parents. In our runs, the value of $n$ is $10 \%$ of the population size.

Having both parents selected, we can cross them with the chosen method of crossing: order crossover (OX). This method is well suited for TSP and VRP solutions, because it keeps some of the current route formation intact, meaning good routes can be passed on to new generations. It consists of isolating one random continuous sub-string out of one parent, ranging from 2 to the length of the string minus 1 (length(parent) -1 ), and completing this string with the rest of the other parent positions circularly, starting at the same position as the first parent's sub-string end. An example can be seen in Figure 9. This results in 2 children being created, each with one exact copy of a sub-string of one parent. 
Figure 9 - OX Crossover example.

\begin{tabular}{ccccccccccccc} 
& & \multicolumn{1}{c}{} & \multicolumn{1}{c}{$\mathrm{c}=4$} & \multicolumn{1}{c}{$j=6$} \\
& & & & & & $\downarrow$ & & $\downarrow$ & & & \\
$\mathrm{P} 1$ & $:$ & 1 & 3 & 2 & 6 & 4 & 5 & 9 & 7 & 8 \\
$\mathrm{P} 2$ & $:$ & 3 & 7 & 8 & 1 & 4 & 9 & 2 & 5 & 6 \\
& & & & & & & & & & \\
$\mathrm{C} 1$ & $:$ & 8 & 1 & 9 & 6 & 4 & 5 & 2 & 3 & 7 \\
$\mathrm{C} 2$ & $:$ & 2 & 6 & 5 & 1 & 4 & 9 & 7 & 8 & 3
\end{tabular}

Source: (PRINS, 2004).

The offspring may not always be feasible, since the crossover can lead to two different customers who are served by drone to be adjacent to each other. To fix unfeasible solutions and as well as to improve each child, both children are sent to the Education step.

\subsection{Education}

In this section the Education process is described. Here unfeasible routes are fixed, a new heuristic for determining a drone launch and landing point is applied, and a local search is performed to improve quality. These steps are not done one after another though, as one local move can make drones routes infeasible, therefore, they all occur several times.

Traditional mutation, for example simple customer swaps or similar moves, is not good enough for more complex structures such as VRP routes, especially with the added complexity of time windows and drone service. As shown in (PRINS, 2004), the addition of local search improves greatly the quality of GAs. Our education step contains LS algorithms that improve both truck's and drone's routes, as well as inter and intra routes.

The education step can be summarized with Algorithm 5. This step is performed for all the children, but the algorithm is invoked individually for each one. First, Split (Algorithm 2) is called to recover the routes from the single route chromosome (line 2). Then the child is evaluated to get its current fitness (line 3) and is duplicated for later comparison (line 4). Afterwards, the first inter route neighborhoods are applied in random order: route merge and route split (line 5). We call it the strong inter routes LS because they alter a route dramatically. The route merge algorithm tries to combine two different routes into one single new route by concatenating them (as seen in Figure 11). The split route algorithm tries to separate one route into two different ones (Figure 12). However, all route changes (including the next LS neighborhoods) are only applied if they improve the current solution, and if so, the current solution value is updated. Figure 10 illustrates this concept. 
Figure 10 - Flow graph for the LS process.

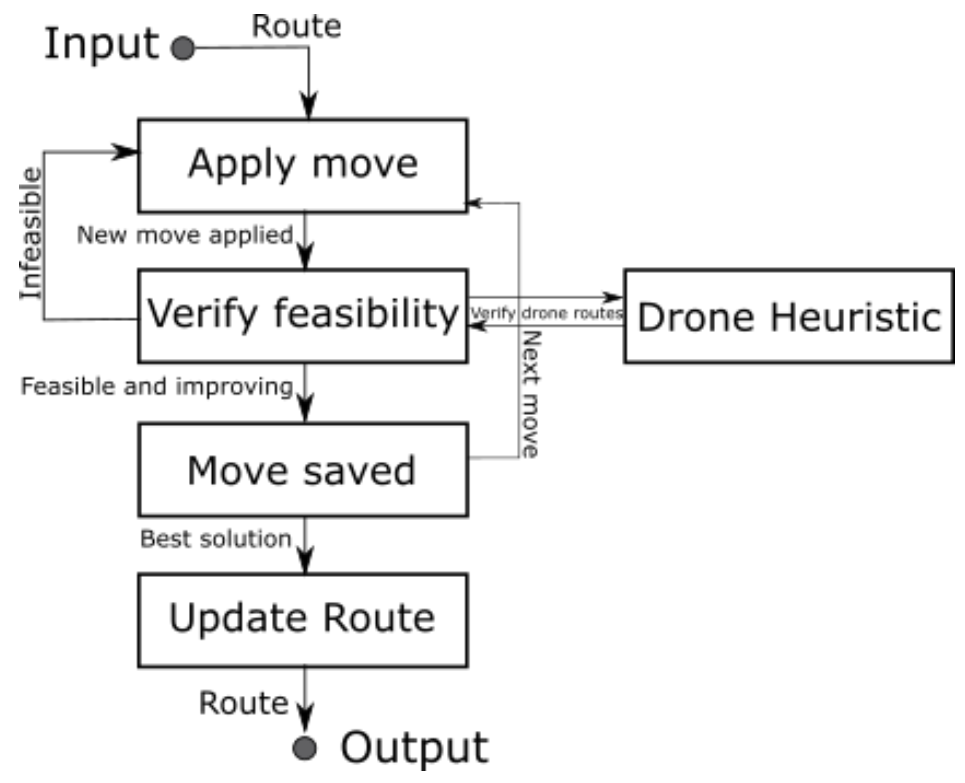

Source: Authors.

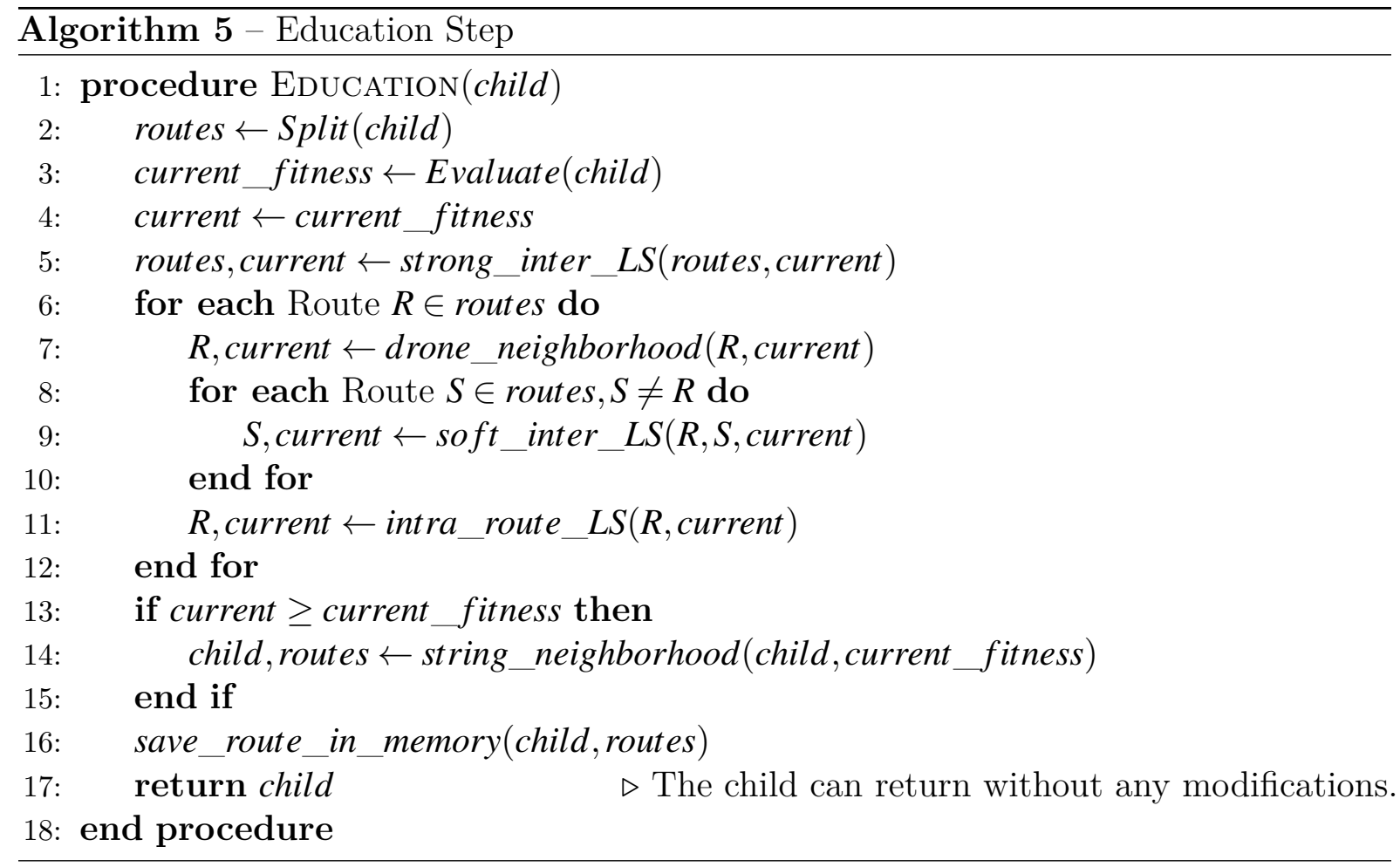

In the next step, we iterate over the routes (line 7) and try to add more droneserved customers (line 8) with a simple LS that tries to serve drone-eligible customers (example in Figure 13), before trying to explore the other inter route neighborhood. The next set of inter route LS try to improve quality by performing simpler swaps. We apply three searches also in random order: the first one, the two-swap*, swaps two consecutive customers from two different routes (Figure 14); the second one, two-swap-exchange*, 
Figure 11 - Merge routes example. Here the route 726 is added to the end of the route 5314.

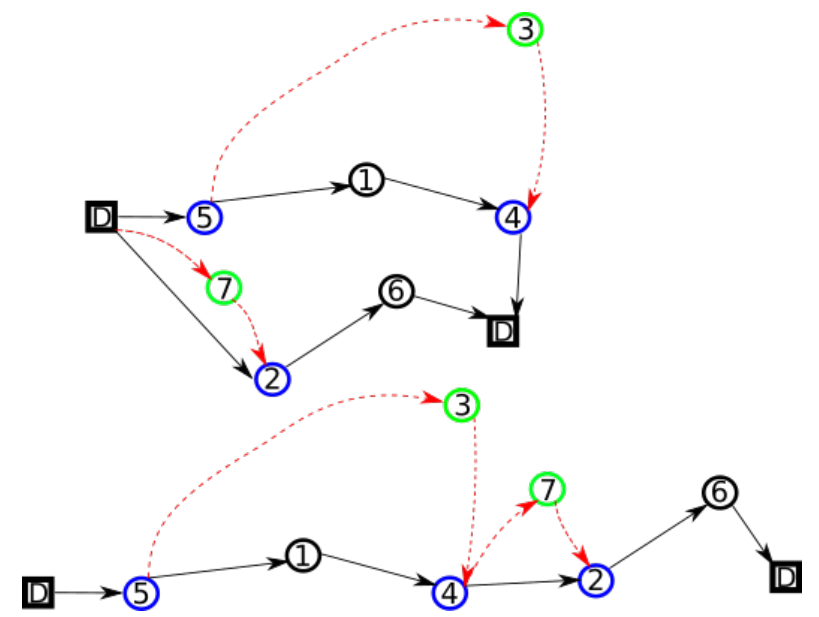

Source: Authors.

Figure 12 - Split routes example. The first route 5314726 is split into two: 531 and 4726
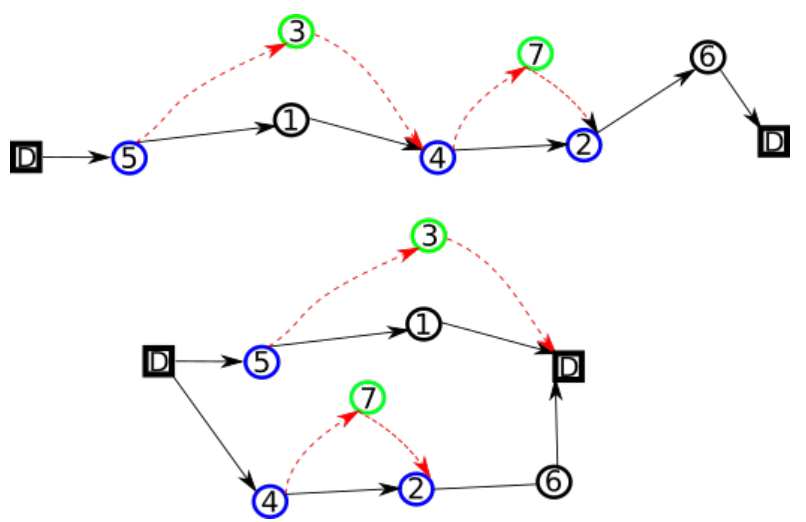

Source: Authors.

is very similar to the two-swap but also changes the customers' order (Figure 15); the third one simply tries to swap two customers between the routes, also known as two-


two-opt, where two customers are swapped (illustrated in Figure 17); a two-swap move, where two consecutive customers swap places with another two consecutive customers (Figure 18); and the last one, two-swap-exchange, works the same way the two-swap, but additionally the customers' have their sequence swapped (Figure 19).

Finally, with all the neighborhoods explored we check whether the new solution is worse than the initial one (line 13). If there was no improvement, i.e. a local minimum, the string neighborhood (line 14) will perform the same changes (also in random order) that the intra-route algorithms did, but considering the whole chromosome instead of just a single route. This is a more expensive (computationally) LS, since we need to check the 
Figure 13 - Drone Local Search example, where the customer 7 is changed from truck-served to drone-served.



Source: Authors.

Figure 14 - An example of the two-swap* move.

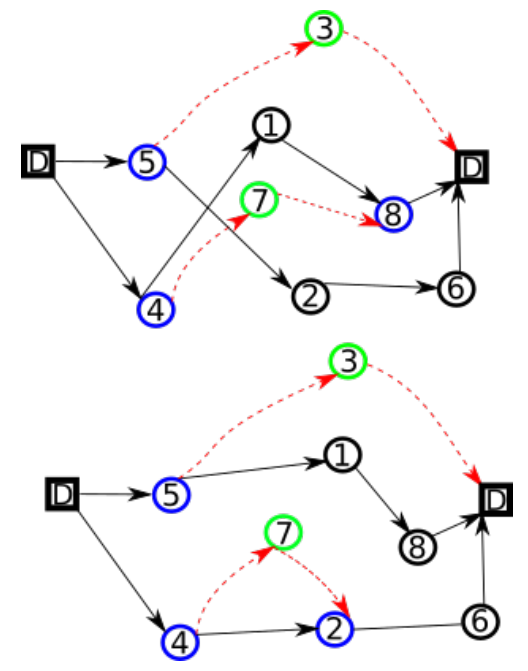

Source: Authors.

feasibility within all routes after any move, but it is a good way to escape local minima and diversify the population when needed. Even so, since most chromosomes will not enter this case, the extra computational cost is barely relevant, and if the child comes out of this step unchanged, then there is likely a chance we have a very strong individual, potentially a global optimum.

The next step for both cases is saving the routes and the child in the local memory. This way, even if the Split algorithm does not return the same routes, we do not lose them. The return of the algorithm is the resulting child chromosome, which may or not have been altered, and can be added to the population in the next steps of the HGA.

Now we present the method which calculates the launching and landing points for 
Figure 15 - An example of the two-swap-exchange* move.

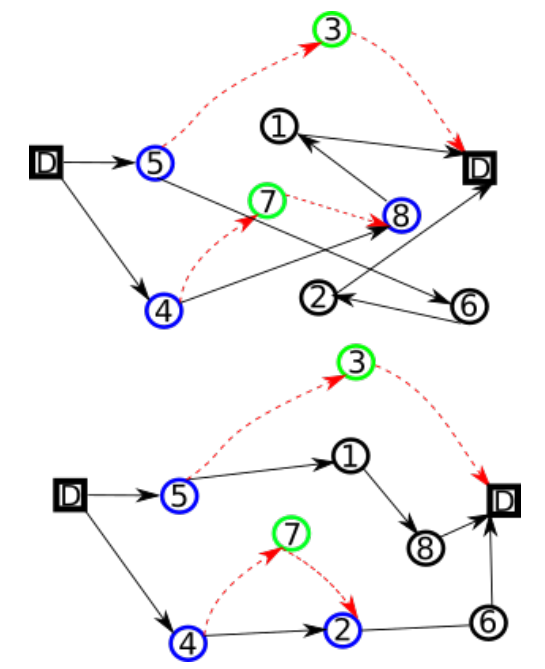

Source: Authors.

Figure 16 - An example of a two-opt* move.


Source: Authors.

each drone.

\subsubsection{Drone Route Method}

Here we introduce our heuristic to find good launching and landing points for the drones. Since we have a chromosome that does not contain that information, each time we evaluate a route we need to calculate those points. And since it is done several times within an execution, it needs to be fast and reliable.

The chosen approach, as shown in Algorithm 6 and illustrated in Figure 20, takes one route as input (line 1) and explore all drone-served customers (line 2). The first 
Figure 17 - An example of a two-opt move.



Source: Authors.

Figure 18 - An example of the two-swap move.
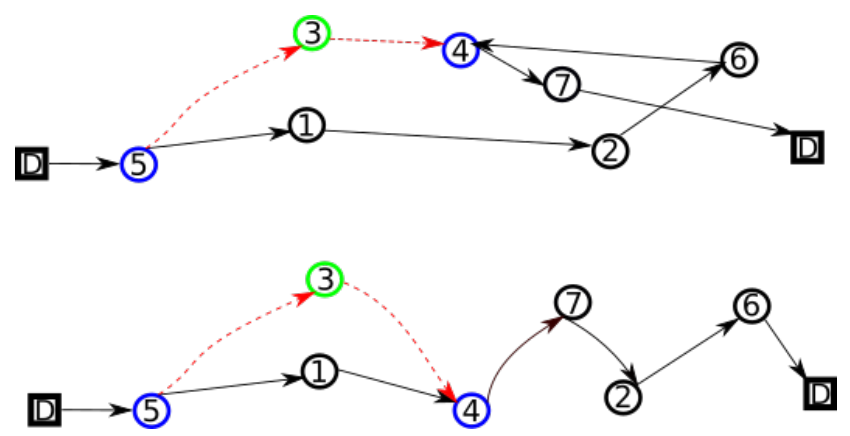

Source: Authors.

Figure 19 - An example of the two-swap-exchange move.
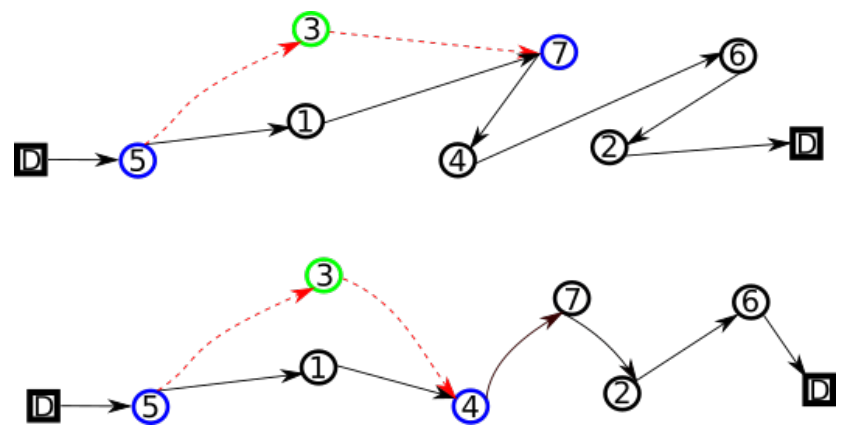

Source: Authors.

customer (highlighted in red in the figure) will have all possible launch and landing sites explored (line 4). First, we calculate if it is feasible for the drone to leave from the previous nodes. In the example from Figure 20, the depot and customer 4 are analyzed. Then. we verify whether the drone can land in any of the later nodes which are not drone-served, customer 3 in the figure. We choose the best feasible route among those to determine the launch and land sites, and since we want to minimize the truck's route, the longest drone's route will reduce the truck's route the most. In the example, we picked the second pair 
$(6 \rightarrow 4 \rightarrow 3)$, and temporarily set the statuses of those two customers as drone launch (line 12) and land (line 13), represented as green zeros in the figure. We repeat this process for the next drone-served customer, in example 2. Both pairs of customers are then explored, $(3,5)$ and $(3,1)$, with the latter being chosen. For the last drone-served customer 7 , the only possibility remaining is the pair $(1,0)$ because the drone cannot depart from client 5 since it already lands on customer 1 . If none of the pairs are feasible, we remove the customer from the drone-served status (line 15).

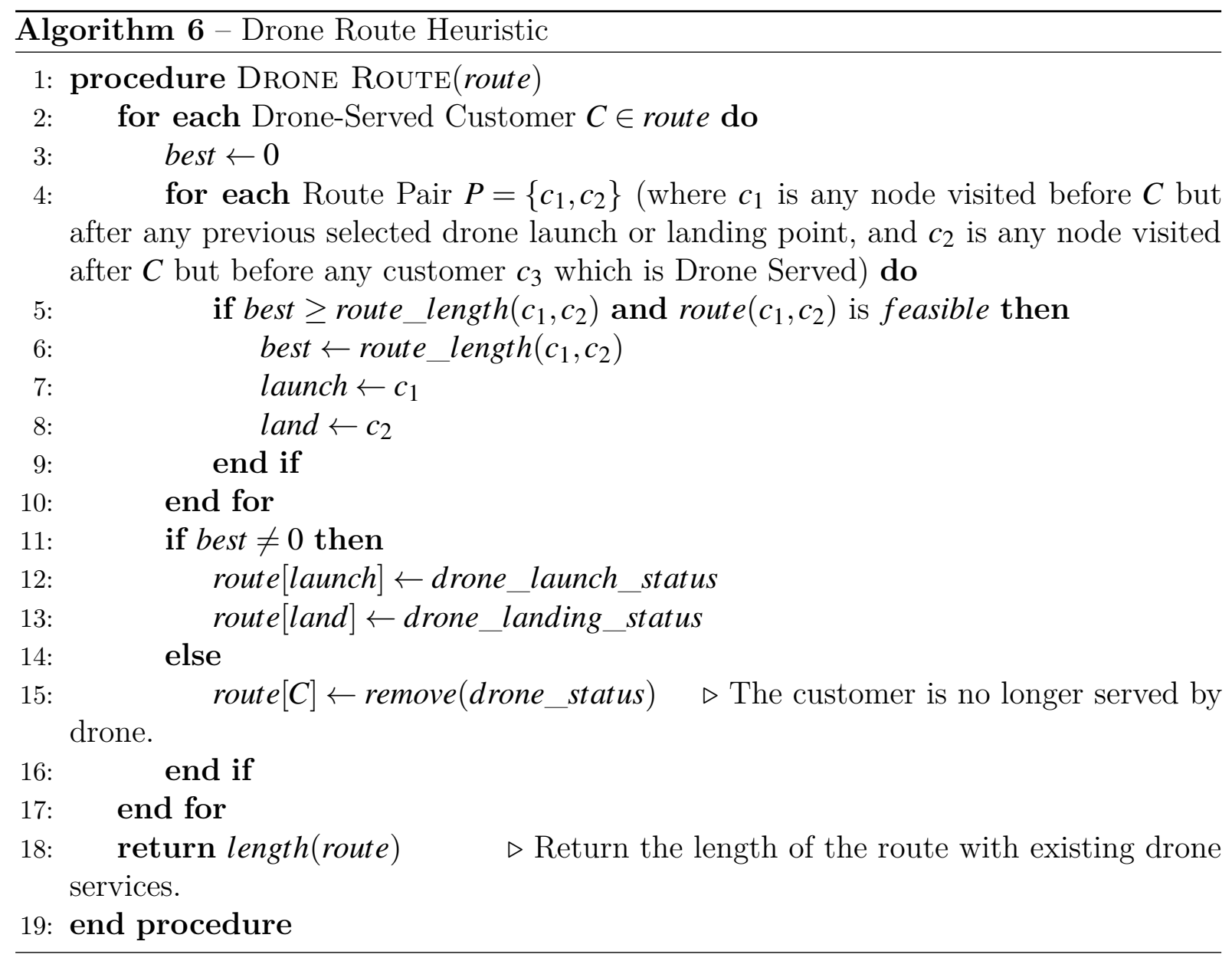


Figure 20 - Drone Heuristic visualization.



Source: Authors. 

CHAPTER

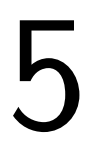

(2)

RESULTS

In this section, the computational experiments with their results are shown. The tests are run in a computer cluster with the following configuration: two Intel Xeon E52680v2 $2.8 \mathrm{GHz}$ processors and $128 \mathrm{~GB}$ of RAM, using a total of up to 40 threads. The MILP model was implemented using Python version 3.5.4 and the IBM CPLEX Python API version 12.8. The HGA is also implemented with the same Python version.

\subsection{Test Instances}

In order to test both the MILP model and the Genetic Algorithm, the Solomon's dataset (SOLOMON, 1987) instances were used. The Solomon's dataset is suitable for testing VRP algorithms that have time-windows, since it varies the length of the timewindow from really tight to more relaxed ones, which are harder to solve. The dateset also has clustered, non-clustered and mixed (both clustered and non-clustered) geographical locations for customers being the classes $\mathrm{C}, \mathrm{R}$ and $\mathrm{RC}$, respectively, which helps to see how do the algorithms behave with such types of customer's location. The class $\mathrm{C}$ has a total of 17 instances, the class $\mathrm{R}$ has a total of 23 and the $\mathrm{RC}$ has 16, each divided into two sub-groups, where the first (aka $\mathrm{C} 1, \mathrm{R} 1$ and $\mathrm{RC} 1$ ) has a shorter time window, whereas the second group (C2, R2 and RC2) has a long schedule horizon.

For all the dataset the number of customers is set to 100 . However, it is very common to divide these into subsets of 25 and 50 customers (taking the first 25 or first 50 customers from the original one, respectively), which work better for more complex versions of the VRPTW. We will be utilizing the 25 subset for testing our proposed MILP, and both the 25 and original 100 for testing the HGA. The next subsections elaborates on the parameters changed overall and the ones specifics to each solution methods. 


\subsubsection{Overall Parameters}

Here the parameters utilized for both the MILP and the HGA are described. All tests are executed with a time limit of 1 hour, not including pre and post processing.

One of the most relevant parameter for testing the VRPD would be the set of drone-eligible customers. Since the Solomon's instances have different weights metrics between each instance type, it was developed the Algorithm 7 to select customers rather than modify all of the instances to fit the particularities of the VRPD (the drones lower weight capacity). On this procedure, the number of customers is not taken into account directly, but rather the number of weights. The procedure takes the list of customers and the chosen percentage in which the calculations will be based upon (line 1). We remove all duplicates weights from the customer list (line 2). And then, by sorting the unique weights of all customers (line 3), each position of the ordered list will cover several customers. The position for each instance is based on the percentage parameter, and by choosing one of these positions (line 4), the set of drone-eligible customers can be defined by selecting every customer that has a parcel with weight less or equal than the one chosen (lines $7-9$ ). For example, for a list of 6 customers' weights $L=[2,2,4,6,11,20]$, the list without duplicates would be $L^{\prime}=[2,4,6,11,20]$, and for a $50 \%$ drone-eligible customer rate, then the procedure from Algorithm 7 would return a list $D=[2,4,6]$, since these are the customers that have a weight equal to or lower than the one in the $50 \%$ position in the list $L^{\prime}$ (rounded-up), in this case, every customer with weight less than or equal to 6 will be selected. This way, the procedure does not depend on the number of customers. In all our tests we use three different drone-eligible values: $25 \%, 50 \%$ and $75 \%$.

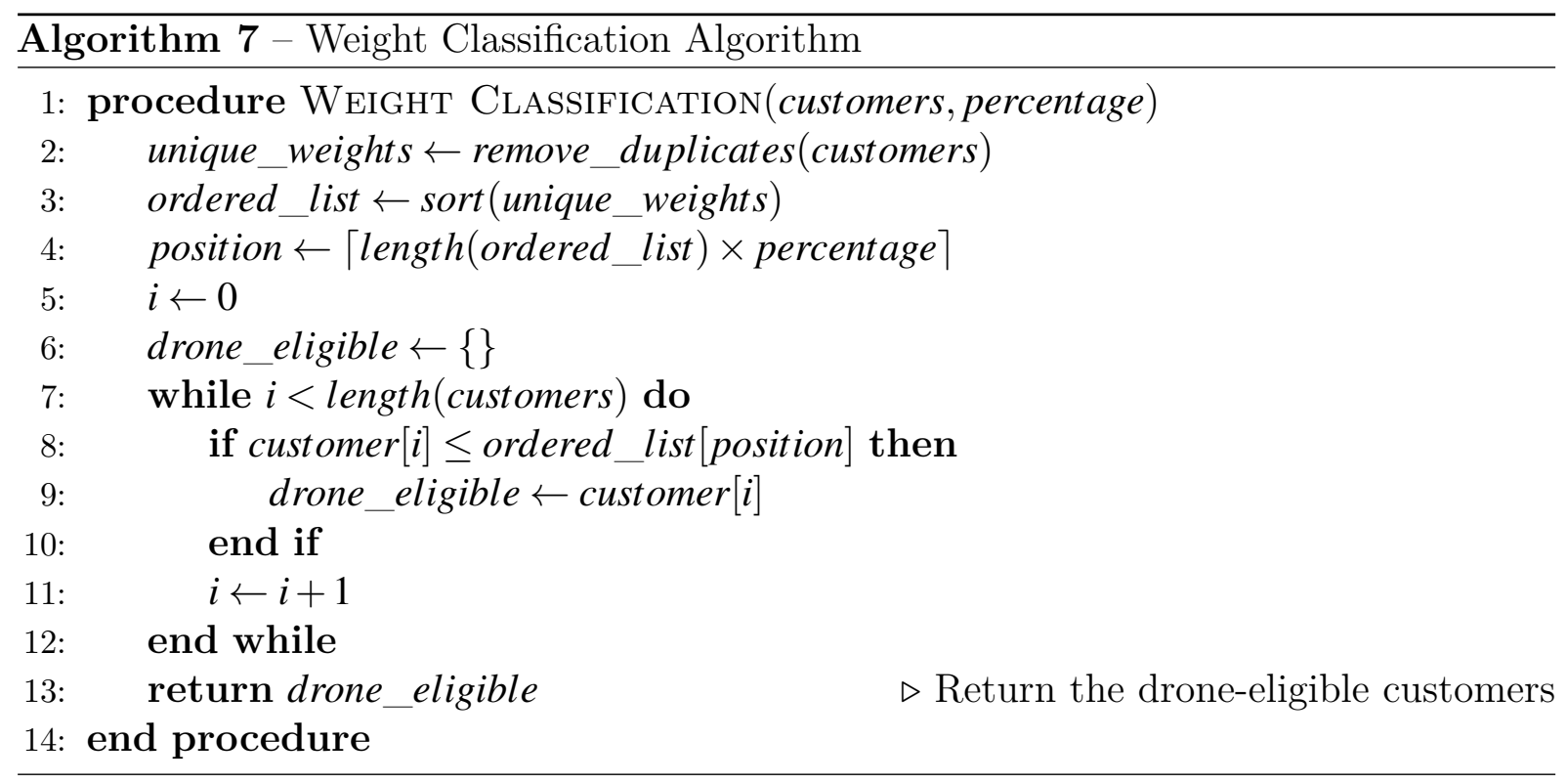

Other important parameters are the travel speed of both the drone and truck. As a general rule, when using Solomon's dataset, the truck speed is set to 1, and all major works utilize that norm. Therefore we also set the truck's speed to 1 and changed the 
drone's speed as a relation to the truck's: half the speed, or 0.5; same speed, also 1; and half as fast, with 1.5 .

As for the drone endurance, since there are different instances types with varying distances between customers, we chose to not use a single endurance value, even though drone models usually have fix flight times. What we did instead was to come up with a formula relating the average distance between customers with an addition to the standard deviation for the distance average, shown in Equation 5.1. Therefore the endurance is dependent on the instance, and can cover a significant amount of customers. This formula when applied to the dataset, had an approximate value to what most commercial drones have which is around 30 minutes of flight, whereas our tests result in value between 25 to 40 units of time, depending on the instance. Additionally, the preparation time for launching $\left(S_{L}\right)$ and for landing $\left(S_{R}\right)$ are set to $10 \%$ of the endurance value.

$$
\text { endurance }=\mu_{\text {distances }}+\sigma_{\text {distances }}
$$

The last parameter is the distance metrics for each type of vehicle. Traditionally, studies use Euclidean distance for the vehicles, since it is a simplification of the real world. And for the sake of comparison, both drones and trucks will be using Euclidean on our tests.

Table 2 - General parameters for the tests. Default parameters are in bold.

\begin{tabular}{c|c}
\hline Parameter & Values \\
\hline Time limit & $3600 s$ \\
\hline Drone eligible weight & $25 \%, \mathbf{5 0} \%, 75 \%$ \\
\hline Drone travel speed & $50 \%, \mathbf{1 0 0} \%, 150 \%$ \\
\hline Drone endurance & $\mu_{\text {distances }}+\sigma_{\text {distances }}$ \\
\hline Distance metrics & Euclidean \\
\hline \multicolumn{2}{c}{ Source - Authors }
\end{tabular}

Table 2 summarizes all test parameters. Since this combination of parameters would require a minimum of 9 runs for all instances (more for the HGA, since we want an average), we defined a default parameter configuration to compare the results. Except for the results of the MILP + HGA, which are run in all possible cases, all other use the following values: $50 \%$ for drone eligible weight and $100 \%$ for drone speed. In the MILP we also use inequation 4.1 to avoid creating unnecessary variables.

\subsubsection{CPLEX Parameters}

The parameters used for solving the MILP were not set to the default values and, hence, we explain them here. The first parameter set was the number of threads used 
for computation. As we had available a robust cluster, we set the number of threads to be used for the MILP calculations to be 40. To improve the high amount of threads usage, we also set an parameter of CPLEX that exploits better concurrency use, i.e. the Opportunistic and Concurrent strategy.

Since the problem was initially taking too much time on node 0 , we also changed the parameter that tries to spend less time on each node by branching based on a pseudo cost algorithm, rather than the exact solution. Additionally, the frequency in which the heuristics were applied was set to 0 , so that to leave the starting node was quicker.

Finally, since to get any feasible solution was hard for a lot of instances, we set the emphasis in feasibility, rather than optimality.

\subsubsection{Hybrid Genetic Parameters}

As described in Chapter 4, the HGA contains few parameters that can be changed. The main ones are the number of generations and the population size. We set these as a function of the number of customers, being the generations six times the amount, and the population with the same size. We summarize the parameters and their value in Table 3.

Table 3 - Parameters for the HGA.

\begin{tabular}{c|c}
\hline Parameter & Value \\
\hline Time limit & $3600 s$ \\
\hline Population Size & $n$ \\
\hline Max Population Size & $n \times 2$ \\
\hline Number of Generations & $50 \%$ \\
\hline Drone served (Random Chromosome) & $T L$ or Generations Limit \\
\hline Stop Criteria &
\end{tabular}

The other parameter present in Table 3 is the chance in which the customers have to be served by drone, that our Algorithm 4 uses. Unlike other GAs, there is no mutation parameter, as we always perform the education step for the offspring.

\subsection{MILP Results}

In this section we present the results for the MILP formulation. There were two approaches to find feasible solutions to the problem. The first one utilizes the Sweep algorithm (Algorithm 3) to find truck-only routes and then CPLEX would improve the routes and add the drones. The second approach was to utilize the result of the HGA (Algorithm 1) to start with a good solution as a boost for the solver. It is important to 
note that without the initial solution given, the CPLEX would reach time limit without any feasible solutions for most instances.

We present the results in the following scheme: the instance name, which are the original Solomon instance names added by a 25 after the instance identifier, since all MILP results are only available to these instances; the objective function Equation 3.1; the GAP given by CPLEX; the dual objective; execution time in seconds; number of explored nodes during execution; the number of variables in the problem; and the number of constraints generated.

Table 4 - Results for the class of instances C, using the Sweep Algorithm as a starting solution.

\begin{tabular}{l|c|c|c|c|c|c|c}
\hline Instance & $\begin{array}{c}\text { Objective } \\
\text { Function }\end{array}$ & Dual & GAP $(\%)$ & Exec. Time & Nodes & Variables & Constraints \\
\hline $\mathrm{c} 25101$ & 181.46 & 181.45 & 0.0 & 105.48 & 12672 & 52255 & 522001 \\
\hline $\mathrm{c} 25102$ & 637.95 & 107.57 & 83 & TL & 1 & 70555 & 543926 \\
\hline $\mathrm{c} 25103$ & 502.13 & 79.80 & 84 & TL & 2 & 75805 & 551551 \\
\hline $\mathrm{c} 25104$ & 389.05 & 79.64 & 80 & TL & 0 & 81555 & 558176 \\
\hline $\mathrm{c} 25105$ & 183.64 & 139.18 & 24 & TL & 95215 & 57705 & 528126 \\
\hline $\mathrm{c} 25106$ & 177.90 & 177.90 & 0.0 & TL & 26929 & 53805 & 523651 \\
\hline $\mathrm{c} 25107$ & 418.45 & 107.37 & 74 & TL & 44975 & 58780 & 529701 \\
\hline $\mathrm{c} 25108$ & 469.70 & 26.00 & 94 & TL & 6841 & 64480 & 536776 \\
\hline $\mathrm{c} 25109$ & 436.62 & 22.77 & 95 & TL & 1 & 69380 & 542951 \\
\hline $\mathrm{c} 25202$ & 201.87 & 201.87 & 0.0 & 297.93 & 65329 & 53905 & 524251 \\
\hline $\mathrm{c} 25203$ & 589.37 & 96.01 & 84 & TL & 9 & 63405 & 537376 \\
\hline $\mathrm{c} 25204$ & 461.24 & 53.52 & 90 & TL & 1 & 71830 & 547951 \\
\hline $\mathrm{c} 25205$ & 547.23 & 97.57 & 82 & TL & 49671 & 59705 & 552576 \\
\hline $\mathrm{c} 25206$ & 438.65 & 46.10 & 89 & TL & 0 & 62105 & 531001 \\
\hline $\mathrm{c} 25207$ & 445.57 & 54.72 & 88 & TL & 1 & 66880 & 539001 \\
\hline Average & - & - & 67 & - & - & 64840.29 & 537711.29 \\
\hline
\end{tabular}

\subsubsection{MILP + Sweep}

The first results are from the MILP execution with the Sweep algorithm providing the initial solution. Table 4, Table 5 and Table 6 summarize the data from the 3 classes, $\mathrm{C}, \mathrm{R}$ and RC, respectively. As seen in the tables, the number of variables and constraints is large for an instance of just 25 customers, averaging around 60000 variables and 530000 constraints, which reflects in the large gaps present. The excess of "Big M" constraints also complement to the great discrepancy in the dual bound. Overall, the model is hard to solve, with many instances not leaving the root node in the time limit of 1 hour.

Table 7, Table 8 and Table 9 compares the results from the MILP, with the Sweep algorithm providing an initial solution, with the optimal value from the VRPTW. As seen, the sweep is not a good starting point for the VRPD. Most values are far worse than the VRPTW optima, where the solutions from classes $\mathrm{C}$ and $\mathrm{RC}$ are on average more than the double of the optimal. Although this comparison is unfair, since the VRPD are 
Table 5 - Results for the class of instances R, using the Sweep Algorithm as a starting solution.

\begin{tabular}{l|c|c|c|c|c|c|c}
\hline Instance & $\begin{array}{c}\text { Objective } \\
\text { Function }\end{array}$ & Dual & GAP $(\%)$ & Exec. Time & Nodes & Variables & Constraints \\
\hline r25101 & 515.19 & 515.19 & 0.0 & 59.77 & 34658 & 31680 & 490126 \\
\hline r25102 & 773.97 & 202.38 & 74 & TL & 102 & 42180 & 505426 \\
\hline r25103 & 678.39 & 182.06 & 73 & TL & 12 & 46730 & 512751 \\
\hline r25104 & 582.78 & 167.97 & 71 & TL & 1 & 49730 & 517126 \\
\hline r25105 & 390.39 & 390.38 & 0.0 & 1055.45 & 27032 & 37130 & 497501 \\
\hline r25106 & 782.37 & 202.38 & 74 & TL & 5 & 45030 & 509501 \\
\hline r25107 & 617.44 & 182.06 & 71 & TL & 1 & 47880 & 514551 \\
\hline r25109 & 594.70 & 167.97 & 72 & TL & 1 & 50230 & 518101 \\
\hline r25110 & 563.29 & 227.88 & 67 & TL & 4477 & 41030 & 504176 \\
\hline r25111 & 653.14 & 175.23 & 69 & TL & 6 & 47455 & 513551 \\
\hline r25112 & 522.93 & 167.97 & 72 & TL & 5 & 46780 & 512851 \\
\hline r25201 & 449.79 & 333.13 & 26 & TL & 2 & 51680 & 520776 \\
\hline r25202 & 635.44 & 202.38 & 68 & TL & 60396 & 39505 & 502326 \\
\hline r25203 & 612.00 & 182.06 & 70 & TL & 45 & 46505 & 512401 \\
\hline r25204 & 536.87 & 167.97 & 69 & TL & 87 & 51005 & 516926 \\
\hline r25205 & 565.43 & 228.94 & 60 & TL & 752 & 43405 & 508626 \\
\hline r25206 & 579.00 & 178.29 & 69 & TL & 10 & 48130 & 515201 \\
\hline r25207 & 548.74 & 167.97 & 69 & TL & 32 & 49655 & 517826 \\
\hline r25208 & 533.49 & 167.97 & 69 & TL & 4 & 51180 & 519901 \\
\hline r25209 & 512.67 & 192.50 & 62 & TL & 218 & 46830 & 513401 \\
\hline r25210 & 605.60 & 209.00 & 65 & TL & 147 & 47380 & 514301 \\
\hline r25211 & 475.78 & 167.97 & 65 & TL & 3 & 51705 & 520826 \\
\hline Average & - & - & 65 & - & - & 46170.22 & 514419.75 \\
\hline
\end{tabular}

mostly not in their optimal and all VRPTW are. Nevertheless, some instances still have an improvement, showing the potential of the application. Given the poor performance with a bad initial solution, we can assume that such simplistic heuristics is not enough for such a complex model. Hence a more elaborated method, such as our proposed HGA, is necessary to find good solutions. We show those results next.

\subsubsection{MILP + HGA}

The next set of results, Table 10, Table 11 and Table 12, show the same tests aforementioned, but with the HGA providing the initial solution. Even though there is still a large gap to the lower bound (dual), overall solutions improved, which becomes more noticeable when comparing to the VRPTW. This comparison is shown in Table 13, Table 14 and Table 15, where we see that in fact there is a reduction in cost (distance), averaging a decrease of around 7\%, despite several of the VRPD solutions not being the optimal. It is important to note that since we are minimizing total distance traveled by the trucks, by using drones to serve some customers, then it is expected that the VRPD will outperform the classical VRPTW. Our goal is to show by how much it does.

The improvements for these instances are also less significant because there is little room for improvement with fewer customers and fewer possible routes. In the next section 
Table 6 - Results for the class of instances RC, using the Sweep Algorithm as a starting solution.

\begin{tabular}{|c|c|c|c|c|c|c|c|}
\hline Instance & $\begin{array}{l}\text { Objective } \\
\text { Function }\end{array}$ & Dual & GAP $(\%)$ & Exec. Time & Nodes & Variables & Constraints \\
\hline rc25101 & 869.38 & 151.38 & 83 & TL & 4 & 49880 & 515826 \\
\hline $\begin{array}{lrc}\mathrm{rc} 25102 \\
\end{array}$ & 764.73 & 47.07 & 94 & TL & 2 & 61055 & 531101 \\
\hline $\mathrm{rc} 25103$ & 622.29 & 38.05 & 94 & TL & 1 & 67955 & 540626 \\
\hline rc25104 & 573.94 & 29.73 & 95 & TL & 0 & 71705 & 545526 \\
\hline rc25105 & 875.21 & 48.59 & 94 & TL & 1 & 58630 & 526951 \\
\hline rc25106 & 695.34 & 45.93 & 93 & TL & 1 & 59580 & 528326 \\
\hline rc25107 & 747.24 & 29.68 & 96 & TL & 0 & 69805 & 541701 \\
\hline rc25108 & 587.71 & 29.68 & 95 & TL & 0 & 73455 & 547926 \\
\hline rc25201 & 722.92 & 187.89 & 74 & TL & 26791 & 53230 & 522301 \\
\hline rc25202 & 794.62 & 47.08 & 94 & $\mathrm{TL}$ & 11 & 62905 & 535026 \\
\hline rc25203 & 738.73 & 39.54 & 95 & TL & 1 & 69680 & 543651 \\
\hline rc25204 & 519.67 & 29.74 & 94 & TL & 3 & 72655 & 547401 \\
\hline $\mathrm{rc} 25205$ & 738.90 & 125.18 & 83 & TL & 2 & 61180 & 532326 \\
\hline rc25206 & 583.21 & 53.54 & 91 & TL & 2 & 61130 & 532501 \\
\hline $\mathrm{rc} 25207$ & 539.85 & 33.54 & 94 & TL & 0 & 68830 & 541926 \\
\hline rc25208 & 454.11 & 29.68 & 93 & TL & 0 & 74505 & 549826 \\
\hline Average & - & 91 & - & - & - & 64761.25 & 536433.81 \\
\hline
\end{tabular}

Table 7 - A comparison between the result of the MILP with Sweep as initial solution and the optima from the VRPTW for the class C.

\begin{tabular}{l|c|c|c}
\hline Instance & MILP + Sweep & VRPTW Optimal & GAP (\%) \\
\hline $\mathrm{c} 25101$ & 181.46 & 191.3 & -5.14 \\
\hline $\mathrm{c} 25102$ & 637.95 & 190.3 & 235.23 \\
\hline $\mathrm{c} 25103$ & 502.13 & 190.3 & 163.86 \\
\hline $\mathrm{c} 25104$ & 389.05 & 186.9 & 108.16 \\
\hline $\mathrm{c} 25105$ & 183.64 & 191.3 & -4.00 \\
\hline $\mathrm{c} 25106$ & 177.9 & 191.3 & -7.00 \\
\hline $\mathrm{c} 25107$ & 418.45 & 191.3 & 118.74 \\
\hline $\mathrm{c} 25108$ & 469.7 & 191.3 & 145.53 \\
\hline $\mathrm{c} 25109$ & 436.62 & 191.3 & 128.24 \\
\hline $\mathrm{c} 25201$ & 201.87 & 214.7 & -5.98 \\
\hline $\mathrm{c} 25202$ & 589.37 & 214.7 & 174.51 \\
\hline $\mathrm{c} 25204$ & 550.19 & 214.7 & 156.26 \\
\hline $\mathrm{c} 25205$ & 461.24 & 213.1 & 116.44 \\
\hline $\mathrm{c} 25206$ & 547.23 & 214.7 & 154.88 \\
\hline $\mathrm{c} 25207$ & 438.65 & 214.7 & 104.31 \\
\hline $\mathrm{c} 25208$ & 398.57 & 214.5 & 107.72 \\
\hline Average & - & 214.5 & 85.70 \\
\hline
\end{tabular}

we show that with more customers, the improvements are more substantial. Table 16 compares how the change in speed affects the performance of the model. We see that with greater speeds better results, which is expected, since faster drones can cross the same distances in less time, allowing for more possible routes. While the other way around works just the opposite: a slower drone will barely benefit the overall distance. It is good to remember that these results are not from optimal values, therefore, in the worst best case (where we have all optimum), the optimal route would have a gap of $0 \%$, since the trucks would perform the same route if the drones would not improve anything. This is 
Table 8 - A comparison between the result of the MILP with Sweep as initial solution and the optima from the VRPTW for the class R.

\begin{tabular}{l|c|c|c}
\hline Instance & MILP + Sweep & VRPTW Optimal & GAP (\%) \\
\hline $\mathrm{r} 25101$ & 515.19 & 617.1 & -16.51 \\
\hline $\mathrm{r} 25102$ & 773.97 & 547.1 & 41.47 \\
\hline $\mathrm{r} 25103$ & 678.39 & 454.6 & 49.23 \\
\hline $\mathrm{r} 25104$ & 582.78 & 416.9 & 39.79 \\
\hline $\mathrm{r} 25105$ & 390.39 & 530.5 & -26.41 \\
\hline $\mathrm{r} 25106$ & 782.37 & 465.4 & 68.11 \\
\hline $\mathrm{r} 25107$ & 617.44 & 424.3 & 45.52 \\
\hline $\mathrm{r} 25108$ & 594.7 & 397.3 & 49.69 \\
\hline $\mathrm{r} 25109$ & 689.29 & 441.3 & 56.20 \\
\hline $\mathrm{r} 25110$ & 563.54 & 444.1 & 26.89 \\
\hline $\mathrm{r} 25111$ & 653.14 & 428.8 & 52.32 \\
\hline $\mathrm{r} 25112$ & 522.93 & 393 & 33.06 \\
\hline $\mathrm{r} 25201$ & 449.79 & 463.3 & -2.92 \\
\hline $\mathrm{r} 25202$ & 635.44 & 410.5 & 54.80 \\
\hline $\mathrm{r} 25203$ & 612 & 391.4 & 56.36 \\
\hline $\mathrm{r} 25204$ & 536.87 & 355 & 51.23 \\
\hline $\mathrm{r} 25205$ & 565.43 & 393 & 43.88 \\
\hline $\mathrm{r} 25206$ & 579 & 374.4 & 54.65 \\
\hline $\mathrm{r} 25208$ & 548.74 & 361.6 & 51.75 \\
\hline $\mathrm{r} 25209$ & 533.49 & 328.2 & 62.55 \\
\hline $\mathrm{r} 25210$ & 512.67 & 370.7 & 38.30 \\
\hline $\mathrm{r} 25211$ & 605.6 & 404.6 & 49.68 \\
\hline Average & 475.78 & 350.9 & 35.59 \\
\hline & - & - & \\
\hline
\end{tabular}

true even for slower drones. The drawback from the increase in speed, is a significant increase in model complexity, as it will have more variables.

On the other hand the drone eligible weight parameter does not seem to change the results by a significant amount, as seen in Table 17. Even though the addition of new customers may also add new route possibilities for the drones, the problem also becomes more hard to solve due to the addition of new variables, so the optimal values could have an slight improvement with higher percentage of customers. For all other instance configurations (shown in Table 2) the results are available at Appendix B. The instances with 100 customers, however, are not solvable by the MILP model, since the system run out of memory beforehand. In the next section we present the results for such instances by using our HGA.

\subsection{HGA Results}

This section presents the results obtained with our Hybrid Genetic Algorithm. All Solomon instances were run in their original size, with 100 customers, and the designed default parameters to the VRPD (presented previously in subsection 5.1.1: 50\% drone eligible weight; and $\mathbf{1 0 0 \%}$ speed of drone when compared to the truck). Table 18, Table 19 
Table 9 - A comparison between the result of the MILP with Sweep as initial solution and the optima from the VRPTW for the class RC.

\begin{tabular}{l|c|c|c}
\hline Instance & MILP + Sweep & VRPTW Optimal & GAP (\%) \\
\hline rc25101 & 869.38 & 461.1 & 88.54 \\
\hline $\mathrm{rc} 25102$ & 764.73 & 351.8 & 117.38 \\
\hline $\mathrm{rc} 25103$ & 622.29 & 333.1 & 86.82 \\
\hline $\mathrm{rc} 25104$ & 573.94 & 306.6 & 87.20 \\
\hline $\mathrm{rc} 25105$ & 875.21 & 411.3 & 112.79 \\
\hline $\mathrm{rc} 25106$ & 695.34 & 345.5 & 101.26 \\
\hline $\mathrm{rc} 25107$ & 747.24 & 298.3 & 150.50 \\
\hline $\mathrm{rc} 25108$ & 587.71 & 294.5 & 99.56 \\
\hline $\mathrm{rc} 25201$ & 722.92 & 360.2 & 100.70 \\
\hline $\mathrm{rc} 25202$ & 794.62 & 338 & 135.09 \\
\hline $\mathrm{rc} 25203$ & 738.73 & 326.9 & 125.98 \\
\hline $\mathrm{rc} 25204$ & 519.67 & 299.7 & 73.40 \\
\hline $\mathrm{rc} 25205$ & 738.9 & 338 & 118.61 \\
\hline $\mathrm{rc} 25206$ & 583.21 & 324 & 80.00 \\
\hline $\mathrm{rc} 25207$ & 539.85 & 298.3 & 80.98 \\
\hline Average & 454.11 & 269.1 & 68.75 \\
\hline
\end{tabular}

Table 10 - Results for the class of instances C, using the HGA as a starting solution.

\begin{tabular}{l|c|c|c|c|c|c|c}
\hline Instance & $\begin{array}{c}\text { Objective } \\
\text { Function }\end{array}$ & Dual & GAP $(\%)$ & Exec. Time & Nodes & Variables & Constraints \\
\hline $\mathrm{c} 25101$ & 181.46 & 181.46 & 0.0 & 257.58 & 21039 & 52255 & 521377 \\
\hline $\mathrm{c} 25102$ & 189.99 & 107.47 & 43 & TL & 5 & 70555 & 543302 \\
\hline $\mathrm{c} 25103$ & 188.12 & 79.61 & 58 & TL & 0 & 75805 & 550927 \\
\hline $\mathrm{c} 25104$ & 182.73 & 79.61 & 56 & TL & 0 & 81555 & 557552 \\
\hline $\mathrm{c} 25105$ & 183.50 & 133.05 & 27 & TL & 9246 & 57705 & 527502 \\
\hline $\mathrm{c} 25106$ & 177.90 & 177.90 & 0.0 & 785.24 & 20137 & 53805 & 523027 \\
\hline $\mathrm{c} 25107$ & 184.10 & 106.66 & 42 & TL & 91 & 58780 & 529077 \\
\hline $\mathrm{c} 25108$ & 184.10 & 31.00 & 83 & TL & 3 & 64480 & 536152 \\
\hline $\mathrm{c} 25201$ & 184.10 & 22.77 & 88 & TL & 1 & 69380 & 542327 \\
\hline $\mathrm{c} 25202$ & 201.87 & 201.87 & 0.0 & 44.92 & 1231 & 53905 & 523627 \\
\hline $\mathrm{c} 25203$ & 205.46 & 96.19 & 53 & TL & 58 & 63405 & 536752 \\
\hline $\mathrm{c} 25204$ & 207.47 & 53.52 & 74 & TL & 2 & 71830 & 547327 \\
\hline $\mathrm{c} 25206$ & 207.57 & 46.91 & 77 & TL & 0 & 75505 & 551952 \\
\hline $\mathrm{c} 25207$ & 205.11 & 47.25 & 53 & TL & 4 & 59705 & 530377 \\
\hline $\mathrm{c} 25208$ & 205.73 & 54.30 & 74 & TL & 1 & 66880 & 539357 \\
\hline Average & - & - & 52 & - & - & 64840.29 & 537087.29 \\
\hline
\end{tabular}

and Table 20 show, for each of the three classes of instances, C, R and RC, respectively, the results from a total of 5 runs. The tables present: the average fitness for all the initial and the final populations, which show that the convergence happens within close grasp from the best solution; the average best solution and how it is compared to the best known solution for the VRPTW ${ }^{1}$; the best solution found in the 5 runs and again how it compares to the VRPTW; and, finally, the average execution time in seconds.

1 Available at <https://www.sintef.no/projectweb/top/vrptw/solomon-benchmark/ 100 - customers /> 
Table 11 - Results for the class of instances R, using the HGA as a starting solution.

\begin{tabular}{|c|c|c|c|c|c|c|c|}
\hline Instance & $\begin{array}{l}\text { Objective } \\
\text { Function }\end{array}$ & Dual & GAP (\%) & Exec. Time & Nodes & Variables & Constraints \\
\hline $\mathrm{r} 25101$ & 515.19 & 515.19 & 0.0 & 20.39 & 490 & 31680 & 489502 \\
\hline r25102 & 492.87 & 208.23 & 58 & TL & 792 & 42180 & 504802 \\
\hline r25103 & 430.94 & 182.06 & 58 & TL & 1 & 46730 & 512127 \\
\hline r25104 & 391.60 & 167.97 & 57 & TL & 1 & 49730 & 516502 \\
\hline r25105 & 398.99 & 346.28 & 13 & TL & 60980 & 37130 & 496877 \\
\hline r25106 & 439.75 & 202.38 & 54 & TL & 2 & 45030 & 508877 \\
\hline r25107 & 385.65 & 182.06 & 53 & TL & 1 & 47880 & 513927 \\
\hline r25108 & 361.20 & 167.97 & 53 & TL & 3 & 50230 & 517477 \\
\hline r25109 & 379.48 & 223.52 & 41 & TL & 2087 & 41030 & 503552 \\
\hline r25110 & 361.28 & 175.23 & 51 & TL & 1 & 47455 & 512927 \\
\hline r25111 & 385.65 & 182.06 & 53 & TL & 2 & 46780 & 512227 \\
\hline r25112 & 335.70 & 169.03 & 50 & TL & 4 & 51680 & 520152 \\
\hline r25201 & 379.52 & 345.00 & 09 & TL & 74931 & 39505 & 501702 \\
\hline r25202 & 382.74 & 202.38 & 47 & TL & 8 & 46505 & 511777 \\
\hline r25203 & 353.81 & 182.06 & 49 & TL & 1 & 49080 & 516302 \\
\hline r25204 & 334.21 & 167.97 & 50 & TL & 2 & 51005 & 519002 \\
\hline r25205 & 358.38 & 239.08 & 33 & TL & 6449 & 43405 & 507902 \\
\hline r25206 & 329.48 & 178.29 & 46 & TL & 1 & 48130 & 514577 \\
\hline r25207 & 314.74 & 167.97 & 47 & TL & 6 & 49655 & 517202 \\
\hline r25208 & 298.05 & 167.97 & 47 & TL & 0 & 51180 & 519277 \\
\hline r25209 & 316.44 & 192.50 & 39 & TL & 2 & 46830 & 512777 \\
\hline r25210 & 364.39 & 209.00 & 43 & TL & 9 & 47380 & 513677 \\
\hline r25211 & 313.44 & 167.97 & 46 & TL & 1 & 51705 & 520202 \\
\hline Average & - & - & 42 & - & - & 46170.22 & 537087.29 \\
\hline
\end{tabular}

From the results, we can observe that the improvement is more significant, with around $20 \%$ decrease in the total traveled distance. This is very motivational for companies that are interested in drone applications, as a reduction of this caliber to a large fleet can have a tremendous impact economically. This also implicates in significantly less greenhouse gas emissions and probably even for customer satisfaction, with faster deliveries.

Figure 21, Figure 22 and Figure 23 illustrates the convergence of the HGA with one example from each class $(\mathrm{C}, \mathrm{R}$ and $\mathrm{RC}$ ). The part (a) of each figure is the initial population, where we can clearly see the half sweep based and the half random based; in part (b) we see how the final population converged, with the first individuals being the better ones; part (c) is a box plot of the convergence, where the variance is clearly decreasing with each generation; and finally, a line plot $(d)$ that shows the evolution of the average and the best individual. Specially in the part (d) of the graphs, we can see that the HGA evolves the most within the first 100 generations. Therefore, it is possible to have a reasonable to good solution even for cases where there is limited execution time. 
Table 12 - Results for the class of instances RC, using the HGA as a starting solution.

\begin{tabular}{l|c|c|c|c|c|c|c}
\hline Instance & $\begin{array}{c}\text { Objective } \\
\text { Function }\end{array}$ & Dual & GAP $(\%)$ & Exec. Time & Nodes & Variables & Constraints \\
\hline rc25101 & 427.12 & 143.25 & 66 & TL & 4 & 49880 & 515202 \\
\hline $\mathrm{rc} 25102$ & 368.94 & 47.07 & 87 & TL & 0 & 61055 & 530477 \\
\hline $\mathrm{rc} 25103$ & 297.69 & 38.05 & 87 & TL & 1 & 67955 & 540002 \\
\hline $\mathrm{rc} 25104$ & 276.32 & 29.73 & 89 & TL & 0 & 71705 & 544902 \\
\hline $\mathrm{rc} 25105$ & 452.32 & 48.59 & 89 & TL & 2 & 58630 & 526327 \\
\hline $\mathrm{rc} 25106$ & 269.41 & 45.93 & 83 & TL & 1 & 59580 & 527702 \\
\hline $\mathrm{rc} 25107$ & 257.94 & 29.68 & 88 & TL & 0 & 69805 & 541077 \\
\hline $\mathrm{rc} 25108$ & 260.16 & 29.68 & 89 & TL & 0 & 73455 & 547302 \\
\hline $\mathrm{rc} 25201$ & 351.48 & 226.79 & 35 & TL & 2416 & 53230 & 521677 \\
\hline $\mathrm{rc} 25203$ & 329.98 & 47.29 & 86 & TL & 9 & 62905 & 534402 \\
\hline $\mathrm{rc} 25204$ & 309.62 & 38.05 & 88 & TL & 1 & 69680 & 543027 \\
\hline $\mathrm{rc} 25205$ & 376.52 & 29.74 & 89 & TL & 0 & 72655 & 546777 \\
\hline $\mathrm{rc} 25206$ & 302.47 & 53.54 & 68 & TL & 1 & 61180 & 531702 \\
\hline $\mathrm{rc} 25207$ & 275.61 & 33.54 & 88 & TL & 1 & 61130 & 531877 \\
\hline $\mathrm{rc} 25208$ & 234.03 & 29.69 & 87 & TL & 0 & 68830 & 541302 \\
\hline Average & - & - & 81 & - & - & 64761.25 & 535809.81 \\
\hline
\end{tabular}

Table 13 - A comparison between the result of the MILP with HGA as initial solution and the optima from the VRPTW for the class C.

\begin{tabular}{l|c|c|c}
\hline Instance & MILP + HGA & VRPTW Optimal & GAP (\%) \\
\hline $\mathrm{c} 25101$ & 181.46 & 191.30 & -5.1 \\
\hline $\mathrm{c} 25102$ & 189.99 & 190.30 & -2.0 \\
\hline $\mathrm{c} 25103$ & 188.12 & 190.30 & -1.1 \\
\hline $\mathrm{c} 25104$ & 182.73 & 186.90 & -2.2 \\
\hline $\mathrm{c} 25105$ & 183.50 & 191.30 & -4.0 \\
\hline $\mathrm{c} 25106$ & 177.90 & 191.30 & -7.0 \\
\hline $\mathrm{c} 25107$ & 184.10 & 191.30 & -3.8 \\
\hline $\mathrm{c} 25108$ & 184.10 & 191.30 & -3.8 \\
\hline $\mathrm{c} 25109$ & 184.10 & 191.30 & -3.8 \\
\hline $\mathrm{c} 25201$ & 201.87 & 214.70 & -6.0 \\
\hline $\mathrm{c} 25202$ & 205.46 & 214.70 & -4.3 \\
\hline $\mathrm{c} 25203$ & 207.47 & 214.70 & -3.4 \\
\hline $\mathrm{c} 25204$ & 206.57 & 213.10 & -3.1 \\
\hline $\mathrm{c} 25205$ & 207.47 & 214.70 & -3.4 \\
\hline $\mathrm{c} 25206$ & 205.11 & 214.70 & -4.5 \\
\hline $\mathrm{c} 25207$ & 205.73 & 214.50 & -4.1 \\
\hline $\mathrm{c} 25208$ & 204.94 & 214.50 & -4.5 \\
\hline Average & - & - & $\mathbf{- 3 . 8 9}$ \\
\hline & & &
\end{tabular}


Table 14 - A comparison between the result of the MILP with HGA as initial solution and the optima from the VRPTW for the class R.

\begin{tabular}{l|c|c|c}
\hline Instance & MILP + HGA & VRPTW Optimal & GAP (\%) \\
\hline r25101 & 515.19 & 617.10 & -16.51 \\
\hline $\mathrm{r} 25102$ & 492.87 & 547.10 & -9.91 \\
\hline $\mathrm{r} 25103$ & 430.94 & 454.60 & -5.20 \\
\hline $\mathrm{r} 25104$ & 391.60 & 416.90 & -6.07 \\
\hline $\mathrm{r} 25105$ & 398.99 & 530.50 & -24.79 \\
\hline $\mathrm{r} 25106$ & 439.75 & 465.40 & -5.51 \\
\hline $\mathrm{r} 25107$ & 385.65 & 424.30 & -9.11 \\
\hline $\mathrm{r} 25108$ & 361.20 & 397.30 & -9.09 \\
\hline $\mathrm{r} 25109$ & 379.48 & 441.30 & -14.01 \\
\hline $\mathrm{r} 25110$ & 361.28 & 444.10 & -18.65 \\
\hline $\mathrm{r} 25111$ & 385.65 & 428.80 & -10.06 \\
\hline $\mathrm{r} 25112$ & 335.70 & 393.00 & -14.58 \\
\hline $\mathrm{r} 25201$ & 379.52 & 463.30 & -18.08 \\
\hline $\mathrm{r} 25202$ & 382.74 & 410.50 & -6.76 \\
\hline $\mathrm{r} 25203$ & 353.81 & 391.40 & -9.60 \\
\hline $\mathrm{r} 25204$ & 334.21 & 355.00 & -5.86 \\
\hline $\mathrm{r} 25205$ & 358.38 & 393.00 & -8.81 \\
\hline $\mathrm{r} 25206$ & 329.48 & 374.40 & -12.00 \\
\hline r25207 & 314.74 & 361.60 & -12.96 \\
\hline r25208 & 298.05 & 328.20 & -9.19 \\
\hline r25209 & 316.44 & 370.70 & -14.64 \\
\hline r25211 & 364.39 & 404.60 & -9.94 \\
\hline Average & 313.44 & 350.90 & -10.68 \\
\hline & - & - & $-\mathbf{1 1 . 3 9}$ \\
\hline & & &
\end{tabular}

Table 15 - A comparison between the result of the MILP with HGA as initial solution and the optima from the VRPTW for the class RC.

\begin{tabular}{l|c|c|c}
\hline Instance & MILP + HGA & VRPTW Optimal & GAP (\%) \\
\hline $\mathrm{rc} 25101$ & 427.12 & 461.10 & -7.4 \\
\hline $\mathrm{rc} 25102$ & 368.94 & 351.80 & 4.87 \\
\hline $\mathrm{rc} 25103$ & 297.69 & 333.10 & -10.6 \\
\hline $\mathrm{rc} 25104$ & 276.32 & 306.60 & -9.9 \\
\hline $\mathrm{rc} 25105$ & 452.32 & 411.30 & 10.0 \\
\hline $\mathrm{rc} 25106$ & 269.41 & 345.50 & -22.0 \\
\hline $\mathrm{rc} 25107$ & 257.94 & 298.30 & -13.5 \\
\hline $\mathrm{rc} 25108$ & 260.16 & 294.50 & -11.7 \\
\hline $\mathrm{rc} 25201$ & 351.48 & 360.20 & -2.4 \\
\hline $\mathrm{rc} 25202$ & 329.98 & 338.00 & -2.4 \\
\hline $\mathrm{rc} 25203$ & 309.62 & 326.90 & -5.3 \\
\hline $\mathrm{rc} 25204$ & 282.72 & 299.7 & -5.67 \\
\hline $\mathrm{rc} 25205$ & 376.56 & 338.0 & 11.4 \\
\hline $\mathrm{rc} 25206$ & 302.47 & 324.0 & -6.6 \\
\hline $\mathrm{rc} 25207$ & 275.61 & 298.3 & -7.6 \\
\hline $\mathrm{rc} 25208$ & 234.03 & 269.1 & -13.03 \\
\hline Average & - & - & -5.74 \\
\hline
\end{tabular}


Table 16 - The effect of changes in speed in the result of the MILP + HGA.

\begin{tabular}{l|c|c|c}
\hline Instance Class & $\begin{array}{c}\text { Speed 50\% } \\
\text { GAP to VRPTW }\end{array}$ & $\begin{array}{c}\text { Speed 100\% } \\
\text { GAP to VRPTW }\end{array}$ & $\begin{array}{c}\text { Speed 150\% } \\
\text { GAP to VRPTW }\end{array}$ \\
\hline $\mathrm{C}$ & $0.61 \%$ & $-3.89 \%$ & $-5.49 \%$ \\
\hline $\mathrm{R}$ & $1.43 \%$ & $-11.39 \%$ & $-18.60 \%$ \\
\hline $\mathrm{RC}$ & $-4.96 \%$ & $-5.74 \%$ & $-7.11 \%$ \\
\hline
\end{tabular}

Table 17 - The effect of changes in drone eligible weight in the result of the MILP + HGA.

\begin{tabular}{l|c|c|c}
\hline Instance Class & $\begin{array}{c}\text { Drone Eligible } \\
\text { W. } 25 \% \\
\text { GAP to VRPTW }\end{array}$ & $\begin{array}{c}\text { Drone Eligible } \\
\text { W. 50\% } \\
\text { GAP to VRPTW }\end{array}$ & $\begin{array}{c}\text { Drone Eligible } \\
\text { W. } 75 \% \\
\text { GAP to VRPTW }\end{array}$ \\
\hline $\mathrm{C}$ & $-2.56 \%$ & $-3.89 \%$ & $-3.81 \%$ \\
\hline $\mathrm{R}$ & $-4.08 \%$ & $-11.39 \%$ & $-13.30 \%$ \\
\hline $\mathrm{RC}$ & $2.76 \%$ & $-5.74 \%$ & $-6.97 \%$ \\
\hline
\end{tabular}

Table 18 - The HGA average for 5 runs and the best of these are compared to the VRPTW best known solutions of the class C. The average initial and final population fitness, and execution time are also shown.

\begin{tabular}{l|c|c|c|c|c|c|c}
\hline Instance & $\begin{array}{c}\text { Avg. Initial } \\
\text { Population }\end{array}$ & $\begin{array}{c}\text { Avg. Final } \\
\text { Population }\end{array}$ & $\begin{array}{c}\text { Avg. } \\
\text { Best }\end{array}$ & $\begin{array}{c}\text { GAP (\%) to } \\
\text { VRPTW } \\
\text { BKS }\end{array}$ & $\begin{array}{c}\text { Overall } \\
\text { Best }\end{array}$ & $\begin{array}{c}\text { GAP (\%)to } \\
\text { VRPTW } \\
\text { BKS }\end{array}$ & $\begin{array}{c}\text { Exec. } \\
\text { Time }\end{array}$ \\
\hline c101 & 4049.22 & 795.59 & 697.44 & $-15.86 \%$ & 694.43 & $-16.23 \%$ & TL \\
\hline c102 & 1603.07 & 388.73 & 355.88 & $-57.07 \%$ & 355.33 & $-57.13 \%$ & 520.92 \\
\hline c103 & 3431.80 & 845.87 & 754.36 & $-8.90 \%$ & 718.36 & $-13.25 \%$ & TL \\
\hline c104 & 3079.21 & 874.77 & 766.87 & $-7.02 \%$ & 741.70 & $-10.07 \%$ & TL \\
\hline c105 & 4032.10 & 812.86 & 726.49 & $-12.36 \%$ & 699.50 & $-15.62 \%$ & TL \\
\hline c106 & 3790.64 & 806.73 & 711.40 & $-14.18 \%$ & 697.12 & $-15.90 \%$ & TL \\
\hline c107 & 3996.90 & 823.48 & 736.24 & $-11.18 \%$ & 715.36 & $-13.70 \%$ & TL \\
\hline c108 & 3604.33 & 811.67 & 715.73 & $-13.66 \%$ & 704.73 & $-14.98 \%$ & TL \\
\hline c109 & 3346.49 & 836.91 & 746.59 & $-9.93 \%$ & 724.92 & $-12.55 \%$ & TL \\
\hline c201 & 4390.46 & 688.64 & 563.85 & $-4.68 \%$ & 521.23 & $-11.89 \%$ & TL \\
\hline c202 & 1661.88 & 364.47 & 336.04 & $-43.19 \%$ & 332.65 & $-43.77 \%$ & 709.36 \\
\hline c203 & 3481.79 & 749.31 & 611.45 & $3.43 \%$ & 588.77 & $-0.41 \%$ & TL \\
\hline c204 & 3107.49 & 822.06 & 688.03 & $16.50 \%$ & 640.29 & $8.41 \%$ & TL \\
\hline c205 & 3844.24 & 654.45 & 537.18 & $-8.78 \%$ & 526.38 & $-10.61 \%$ & TL \\
\hline c206 & 3660.49 & 672.95 & 570.09 & $-3.13 \%$ & 536.74 & $-8.79 \%$ & TL \\
\hline c207 & 3591.80 & 671.65 & 537.41 & $-8.65 \%$ & 496.68 & $-15.57 \%$ & TL \\
\hline c208 & 3517.30 & 665.71 & 557.88 & $-5.17 \%$ & 522.88 & $-11.12 \%$ & TL \\
\hline Average & & & & $-11.99 \%$ & & $-15.48 \%$ & - \\
\hline
\end{tabular}


Table 19 - The HGA average for 5 runs and the best of these are compared to the VRPTW best known solutions of the class R. The average initial and final population fitness, and execution time are also shown.

\begin{tabular}{l|c|c|c|c|c|c|c}
\hline Instance & $\begin{array}{c}\text { Avg. Initial } \\
\text { Population }\end{array}$ & $\begin{array}{c}\text { Avg. Final } \\
\text { Population }\end{array}$ & $\begin{array}{c}\text { Avg. } \\
\text { Best }\end{array}$ & $\begin{array}{c}\text { GAP (\%) to } \\
\text { VRPTW } \\
\text { BKS }\end{array}$ & $\begin{array}{c}\text { Overall } \\
\text { Best }\end{array}$ & $\begin{array}{c}\text { GAP (\%)to } \\
\text { VRPTW } \\
\text { BKS }\end{array}$ & $\begin{array}{c}\text { Exec. } \\
\text { Time }\end{array}$ \\
\hline r101 & 3800.53 & 1450.587 & 1378.90 & $-16.47 \%$ & 1365.15 & $-17.30 \%$ & TL \\
\hline r102 & 1947.18 & 822.6307 & 813.53 & $-45.26 \%$ & 806.51 & $-45.73 \%$ & 338.59 \\
\hline r103 & 3190.85 & 1094.622 & 1007.83 & $-22.04 \%$ & 987.98 & $-23.57 \%$ & TL \\
\hline r104 & 2877.30 & 893.5116 & 824.19 & $-18.18 \%$ & 813.45 & $-19.24 \%$ & TL \\
\hline r105 & 3576.28 & 1181.89 & 1070.65 & $-22.25 \%$ & 1053.52 & $-23.50 \%$ & TL \\
\hline r106 & 3398.87 & 1127.614 & 1016.70 & $-18.80 \%$ & 1000.64 & $-20.08 \%$ & TL \\
\hline r107 & 3062.30 & 977.0588 & 895.88 & $-18.90 \%$ & 884.95 & $-19.89 \%$ & TL \\
\hline r109 & 2832.98 & 863.0353 & 794.32 & $-17.33 \%$ & 763.44 & $-20.55 \%$ & TL \\
\hline r110 & 3247.62 & 1026.56 & 921.73 & $-22.85 \%$ & 886.15 & $-25.83 \%$ & TL \\
\hline r111 & 3055.64 & 947.32 & 854.18 & $-23.66 \%$ & 830.02 & $-25.81 \%$ & TL \\
\hline r112 & 2839.66 & 958.61 & 848.43 & $-22.64 \%$ & 822.21 & $-25.03 \%$ & TL \\
\hline r202 & 1819.86 & 878.48 & 802.51 & $-18.29 \%$ & 776.49 & $-20.94 \%$ & TL \\
\hline r203 & 3166.85 & 1012.62 & 930.44 & $-21.92 \%$ & 912.71 & $-23.41 \%$ & TL \\
\hline r204 & 2941.52 & 887.33 & 800.88 & $-14.75 \%$ & 787.32 & $-16.20 \%$ & TL \\
\hline r205 & 2717.57 & 749.48 & 649.37 & $-21.34 \%$ & 636.72 & $-22.87 \%$ & TL \\
\hline r206 & 2845.62 & 952.95 & 877.25 & $-11.78 \%$ & 848.55 & $-14.67 \%$ & TL \\
\hline r207 & 2749.56 & 887.60 & 801.98 & $-11.49 \%$ & 777.21 & $-14.23 \%$ & TL \\
\hline r208 & 2552.39 & 702.52 & 601.01 & $-17.31 \%$ & 555.30 & $-23.60 \%$ & TL \\
\hline r209 & 2752.94 & 862.21 & 726.42 & $-16.40 \%$ & 625.58 & $-19.97 \%$ & TL \\
\hline r210 & 2872.91 & 880.22 & 785.91 & $-16.34 \%$ & 761.01 & $-18.99 \%$ & TL \\
\hline r211 & 2692.67 & 823.82 & 720.81 & $-17.71 \%$ & 654.79 & $-26.07 \%$ & TL \\
\hline Average & & & & $-20.67 \%$ & & $-23.01 \%$ & - \\
\hline & & & & & & & \\
\hline
\end{tabular}

Table 20 - The HGA average for 5 runs and the best of these are compared to the VRPTW best known solutions of the class RC. The average initial and final population fitness, and execution time are also shown.

\begin{tabular}{|c|c|c|c|c|c|c|c|}
\hline Instance & $\begin{array}{l}\text { Avg. Initial } \\
\text { Population }\end{array}$ & $\begin{array}{l}\text { Avg. Final } \\
\text { Population }\end{array}$ & $\begin{array}{l}\text { Avg. } \\
\text { Best }\end{array}$ & $\begin{array}{c}\text { GAP (\%) to } \\
\text { VRPTW } \\
\text { BKS }\end{array}$ & $\begin{array}{c}\text { Overall } \\
\text { Best }\end{array}$ & $\begin{array}{c}\text { GAP (\%)to } \\
\text { VRPTW } \\
\text { BKS }\end{array}$ & $\begin{array}{l}\text { Exec. } \\
\text { Time }\end{array}$ \\
\hline rc101 & 4520.94 & 1532.99 & 1439.48 & $-15.17 \%$ & 1414.99 & $-16.62 \%$ & TL \\
\hline rc102 & 2667.74 & 793.32 & 690.55 & $-55.58 \%$ & $\begin{array}{l}634.83 \\
\end{array}$ & $-59.17 \%$ & 528.91 \\
\hline rc103 & 4045.26 & 1230.17 & 1115.57 & $-11.58 \%$ & 1089.46 & $-13.65 \%$ & TL \\
\hline $\mathrm{rc104}$ & 3730.75 & 1099.66 & 1004.83 & $-11.51 \%$ & 982.76 & $-13.45 \%$ & TL \\
\hline rc105 & 4487.25 & 1417.07 & 1257.35 & $-22.84 \%$ & 1195.46 & $-26.63 \%$ & TL \\
\hline rc106 & 4031.00 & 1261.29 & 1150.41 & $-19.25 \%$ & 1116.38 & $-21.64 \%$ & TL \\
\hline rc107 & 3886.94 & 1179.04 & 1060.01 & $-13.85 \%$ & 1047.37 & $-14.88 \%$ & TL \\
\hline rc108 & 3817.71 & 1079.67 & 964.30 & $-15.40 \%$ & 938.21 & $-17.69 \%$ & TL \\
\hline rc201 & 4211.00 & 1246.49 & 1151.74 & $-18.14 \%$ & 1128.36 & $-19.80 \%$ & TL \\
\hline rc202 & 2386.24 & 674.57 & 611.15 & $-55.25 \%$ & $\begin{array}{l}578.12 \\
\end{array}$ & $-57.67 \%$ & 629.98 \\
\hline rc203 & 3741.70 & $\begin{array}{l}992.38 \\
\end{array}$ & 878.43 & $-16.31 \%$ & 838.76 & $-20.09 \%$ & TL \\
\hline rc204 & 3450.02 & 870.62 & 751.56 & $-5.87 \%$ & 712.64 & $-10.75 \%$ & TL \\
\hline rc205 & 3928.10 & 1140.34 & 1047.60 & $-19.27 \%$ & 1020.84 & $-21.33 \%$ & TL \\
\hline rc206 & 3909.84 & 1072.14 & 975.96 & $-14.86 \%$ & 944.61 & $-17.60 \%$ & TL \\
\hline rc207 & 3666.33 & 1016.87 & 927.54 & $-12.59 \%$ & 874.05 & $-17.63 \%$ & TL \\
\hline rc208 & 3323.67 & 857.54 & 745.81 & $-9.94 \%$ & 730.65 & $-11.77 \%$ & TL \\
\hline Average & & & & $-19.84 \%$ & & $-22.52 \%$ & - \\
\hline
\end{tabular}




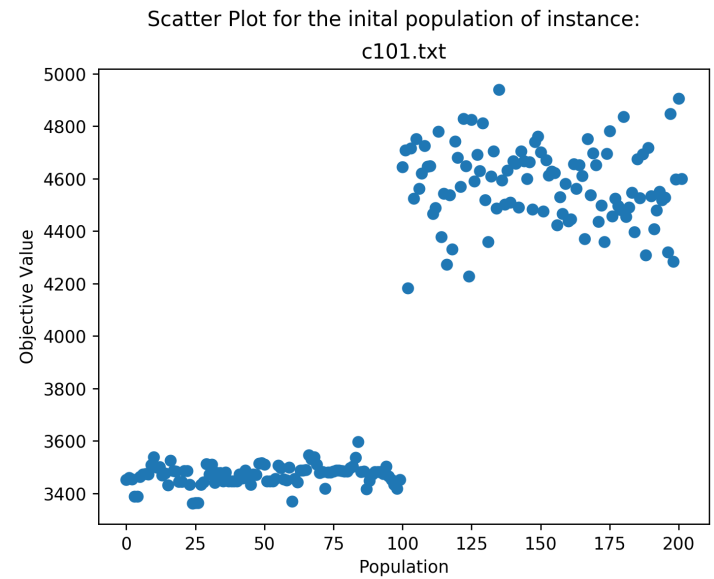

(a) Initial population of the HGA. The left side is

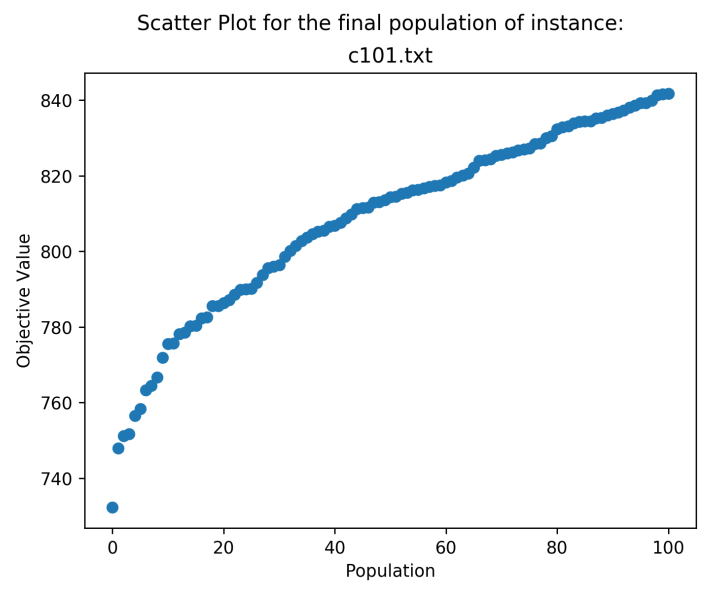

generated with the Sweep algorithm, and the(b) Result of the convergence in the population by right side by a random permutation. the end of execution.

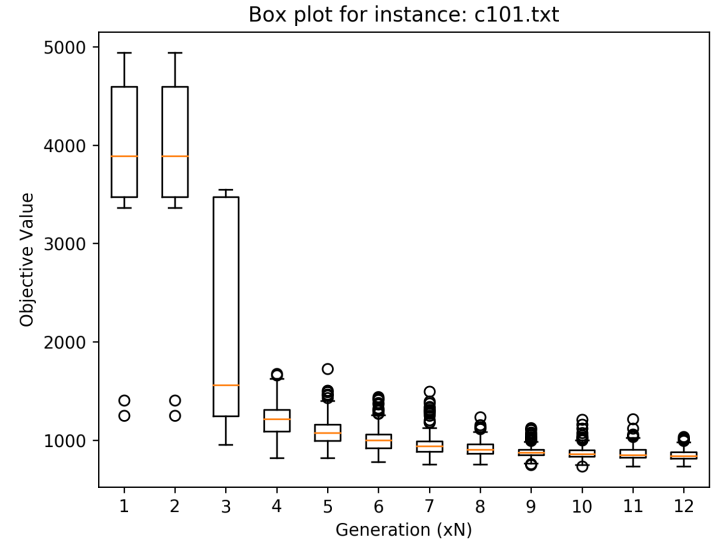

(c) Box plot of the population by generations.

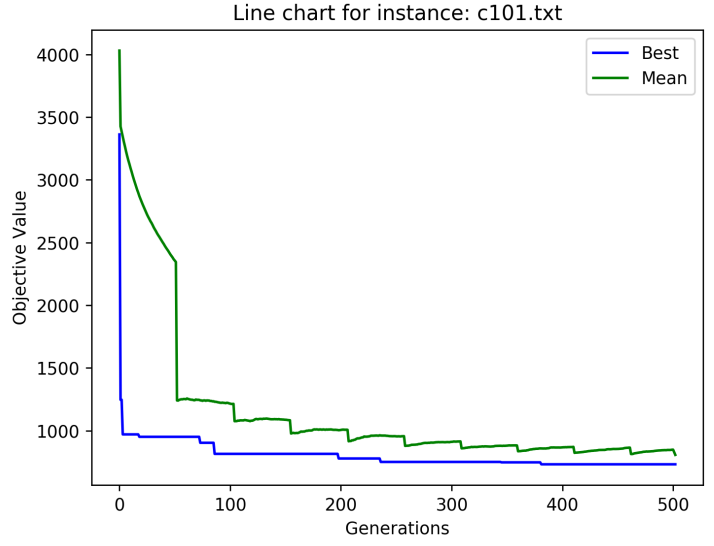

(d) Evolution of population and best individual through generations.

Figure 21 - Illustration of the HGA in execution. Example with instance C101 from (SOLOMON, 1987). 

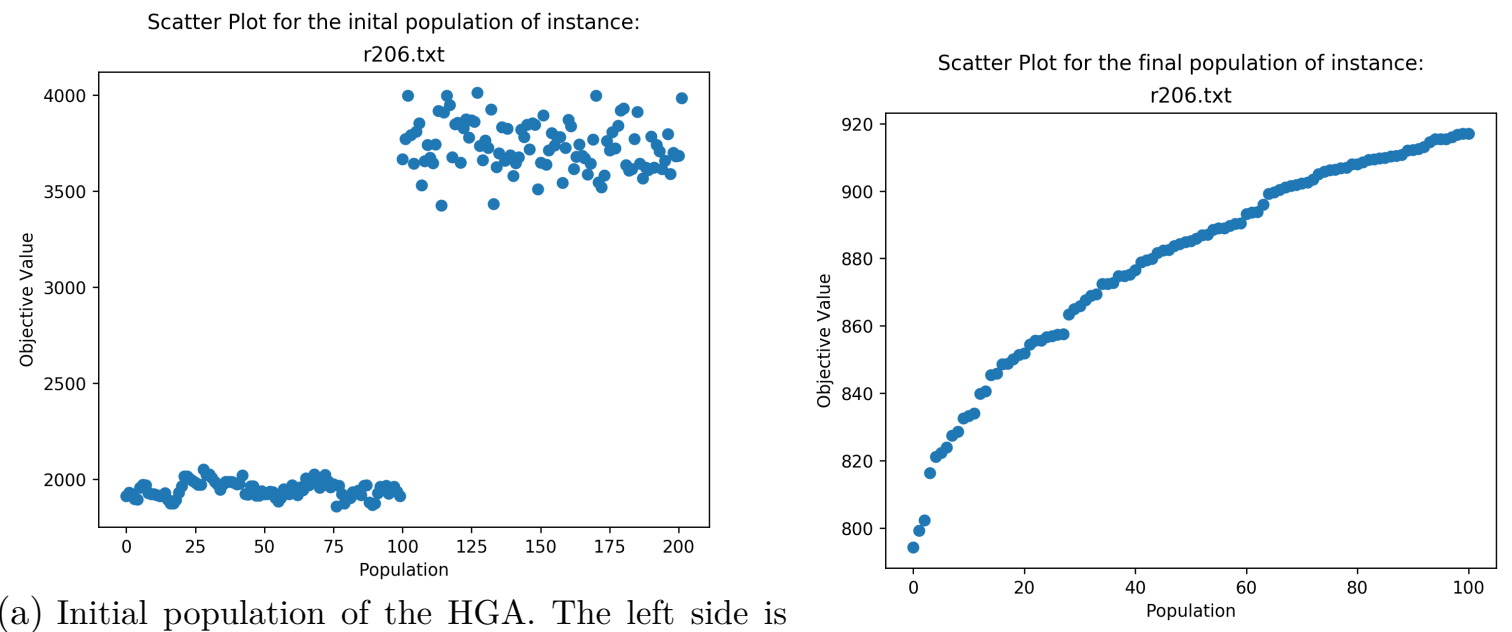

(a) Initial population of the HGA. The left side is generated with the Sweep algorithm, and the(b) Result of the convergence in the population by right side by a random permutation. the end of execution.



(c) Box plot of the population by generations.



(d) Evolution of population and best individual through generations.

Figure 22 - Illustration of the HGA in execution. Example with instance R206 from (SOLOMON, 1987). 

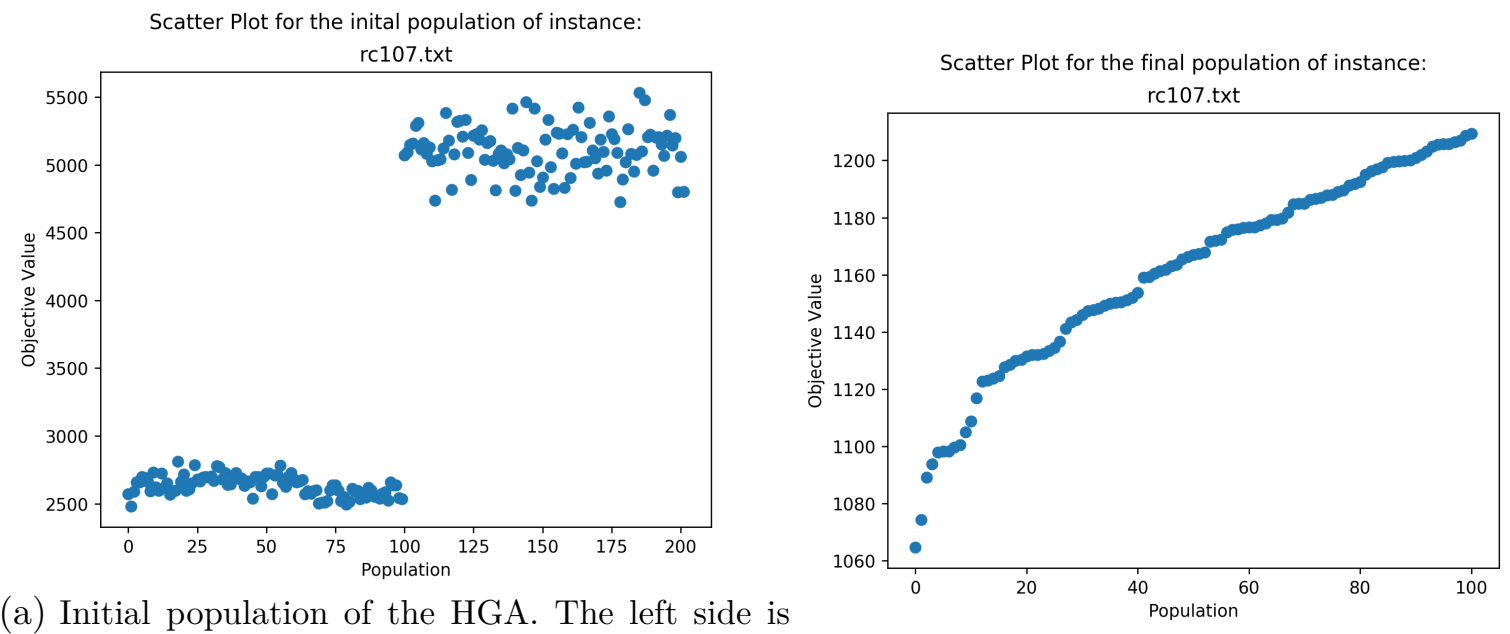

(a) Initial population of the HGA. The left side is generated with the Sweep algorithm, and the(b) Result of the convergence in the population by right side by a random permutation. the end of execution.

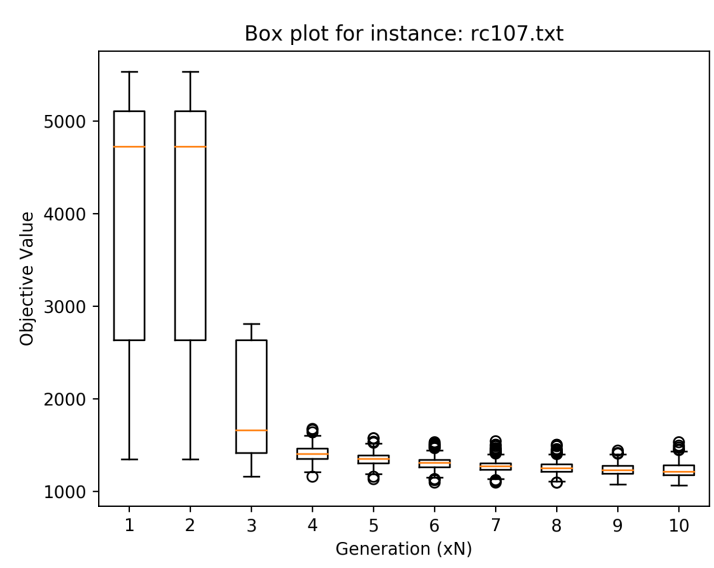

(c) Box plot of the population by generations.

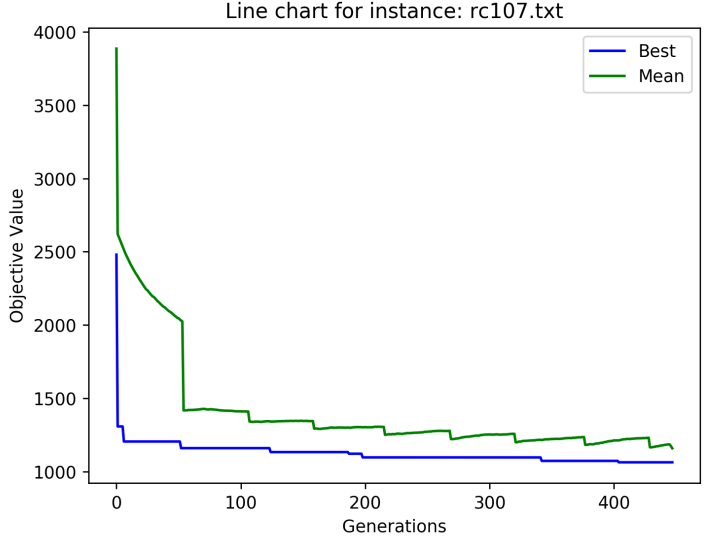

(d) Evolution of population and best individual through generations.

Figure 23 - Illustration of the HGA in execution. Example with instance RC107 from (SOLOMON, 1987). 

CHAPTER

6

FINAL REMARKS

In this dissertation, the Vehicle Routing Problem with Drones is discussed. The problem is considered a very topical discussion, not only in the scientific field but in politics and in general society as well. Several companies are already developing techniques to apply drones to their markets. Although there are complications implied in their use, drones can speed up delivery time and could directly help reduce traffic and greenhouse gas emissions.

We presented a literature review showing the most relevant work in the field of logistics applications considering drones as a form of delivery vehicle. Specifically, we focus on the work that also combines the drones with regular vehicles, in order to exploit the best of the two worlds: the truck's autonomy with the drone's agility.

To solve the problem we proposed two approaches: a Mixed Integer Linear Programming model, although this approach is more theoretical and to serve as a mathematical basis of comparison and structural definition; and a Hybrid Genetic Algorithm, which combines the benefits of a population-based algorithm, i.e exploration, with a local search that tries to find immediate neighbors with better solutions, i.e exploitation. The first approach was based on the model in (MURRAY; CHU, 2015) (discussed in Chapter 3), which was extended for allowing vehicle limited capacity and customers time windows, as well as the additional vehicles. The HGA was based on the idea of (VIDAL et al., 2014). We developed a new chromosome, split and drone procedures, and some neighborhoods for the VRPD, which resulted in a consistent output to a complex problem.

By using the dataset of (SOLOMON, 1987), we tested both approaches to verify their correctness and to measure the benefits of employing drones on the delivery network. The results show that the drones can reduce the distance traversed by trucks to astounding $50 \%$ in some test instances, and on average the reduction is around $20 \%$. This improvement is more notable in the larger tests, however, the small instances still have an 
average gain of 7\%. We also aimed to compare our model with the one in (PUGLIESE; GUERRIERO, 2017), since it is the only other MILP model for the VRPD. However, the model was unable to execute any of the 25 customer instances due to the excessive amount of memory consumption.

The results show the potential of drone driven applications. They are relatively cheap and given the opportunity can benefit a lot of parties: customers with faster deliveries; companies with less expenses; and the environment with fewer gas emission. There is still a lot to discuss and to study before the drones become a reality. We discuss some next steps on this matter next.

\subsection{Future Research}

The application of drones has a wide open field of possibilities. Considering only logistics and the approaches discussed in this work, some are very evident. For example, finding better and more sophisticated neighborhoods for drones routes, as well as a more refined way to determine their routes. One possibility is to find the optimal take-off and landing locations, based on the already fixed truck route. This would require a robust model that can be solved very quickly, but could improve the results significantly.

One obvious addition to the model is to have more then one drone per truck. Our model can be adapted to this, but since it is already hard to solve, adding more indexes to the variables or even more constraints may hinder it to a point where it becomes unsolvable. The proposed HGA can also be adapted, where each additional drone would add one more layer to our chromosome. This can become too complex to handle the local searches, but it is an interesting possibility.

Other research perspectives include: the drones visiting more than one customer before returning to the truck; consider weather to determine the use of drones or other types of stochasticity; more realistic scenarios, such as addition of no-fly zones.

For this work, we want to explore other parameters, such as different distance metrics for each vehicle type; compare to other solution methods in the literature and test with other datasets; evaluate more deeply the impact of each parameter, each neighborhood, and different initial algorithms; do a statistical analysis of the results; and, add energy and battery consumption constraints. 
AGATZ, N.; BOUMAN, P.; SCHMIDT, M. Optimization approaches for the traveling salesman problem with drone. SSRN Electronic Journal, Elsevier BV, 2015. Citations on pages 31,34 , and 37 .

BOUMAN, P.; AGATZ, N.; SCHMIDT, M. Dynamic programming approaches for the traveling salesman problem with drone. SSRN Electronic Journal, Elsevier BV, 2017. Citations on pages 34 and 37.

BRAEKERS, K.; RAMAEKERS, K.; NIEUWENHUYSE, I. V. The vehicle routing problem: State of the art classification and review. Computers \& Industrial Engineering, Elsevier BV, v. 99, p. 300-313, sep 2016. Citation on page 30.

CARLSSON, J. G.; SONG, S. Coordinated logistics with a truck and a drone. Management Science, Institute for Operations Research and the Management Sciences (INFORMS), v. 64, n. 9, p. 4052-4069, sep 2018. Citations on pages 34 and 37.

CBS. Amazon unveils futuristic plan: Delivery by drone. 2013. Available: <https:// www.cbsnews.com/news/amazon-unveils-futuristic-plan-delivery-by-drone/>. Citation on page 25 .

CHANG, Y. S.; LEE, H. J. Optimal delivery routing with wider drone-delivery areas along a shorter truck-route. Expert Systems with Applications, Elsevier BV, v. 104, p. 307-317, aug 2018. Citations on pages 35 and 37.

CHOWDHURY, S.; EMELOGU, A.; MARUfuZZAMAN, M.; NURRE, S. G.; BIAN, L. Drones for disaster response and relief operations: A continuous approximation model. International Journal of Production Economics, Elsevier BV, v. 188, p. 167-184, jun 2017. Citation on page 25.

CHUNG, J.-B. Heuristic method for collaborative parcel delivery with drone. Journal of Distribution Science, Korea Distribution Science Association, v. 16, n. 2, p. 19-24, feb 2018. Citations on pages 35 and 37.

CLARKE, G.; WRIGHT, J. W. Scheduling of vehicles from a central depot to a number of delivery points. Operations Research, Institute for Operations Research and the Management Sciences (INFORMS), v. 12, n. 4, p. 568-581, aug 1964. Citation on page 30.

COSTA, J. G. C.; SANTOS, M. O. d.; SILVA, A. V.; ASSUNçãO, M. A. F. d.; SANTOS, M. O. d. A mixed integer linear programming model for the drone-assisted tsp. In: Simpósio Brasileiro de Pesquisa Operacional - SBPO. [S.l.]: SOBRAPO, 2018. Citations on pages 27 and 113 .

DANTZIG, G. B.; RAMSER, J. H. The truck dispatching problem. Management Science, Institute for Operations Research and the Management Sciences (INFORMS), v. 6, n. 1 , p. 80-91, oct 1959. Citations on pages 29 and 30. 
DHL. DHL parcelcopter launches initial operations for research purposes. 2014. Available: <http://www.dhl.com/en/press/releases/releases_2014/group/ dhl_parcelcopter_launches_initial_operations_for_research_purposes.html $>$. Citation on page 25 .

DORLING, K.; HEINRICHS, J.; MESSIER, G. G.; MAGIEROWSKI, S. Vehicle routing problems for drone delivery. IEEE Transactions on Systems, Man, and Cybernetics: Systems, Institute of Electrical and Electronics Engineers (IEEE), v. 47, n. 1, p. 70-85, jan 2017. Citations on pages 32 and 37.

EKSIOGLU, B.; VURAL, A. V.; REISMAN, A. The vehicle routing problem: A taxonomic review. Computers \& Industrial Engineering, Elsevier BV, v. 57, n. 4, p. 1472-1483, nov 2009. Citation on page 30.

EREN, H.; ÇELIK, m. Risk assessment for aerial package delivery. Zenodo, 2018. Citation on page 27 .

FERRANDEZ, S. M.; HARBISON, T.; WEBER, T.; STURGES, R.; RICH, R. Optimization of a truck-drone in tandem delivery network using k-means and genetic algorithm. Journal of Industrial Engineering and Management, Omnia Publisher SL, v. 9, n. 2, p. 374, apr 2016. Citations on pages 32 and 37.

FREITAS, J. C. de; PENNA, P. H. V. A randomized variable neighborhood descent heuristic to solve the flying sidekick traveling salesman problem. Electronic Notes in Discrete Mathematics, Elsevier BV, v. 66, p. 95-102, apr 2018. Citations on pages 33 and 37.

GILCHRIST, K. World's first drone delivery service launches in Iceland. CNBC, 2017. Available: <https://www.cnbc.com/2017/08/22/ worlds-first-drone-delivery-service-launches-in-iceland.html>. Citation on page 27.

HA, Q. M.; DEVILLE, Y.; PHAM, Q. D.; Hà, M. H. Heuristic methods for the traveling salesman problem with drone. 2015. Citations on pages 32, 35, and 37.

HA, Q. M.; DEVILLE, Y.; PHAM, Q. D.; HA, M. H. On the min-cost traveling salesman problem with drone. Transportation Research Part C: Emerging Technologies, Elsevier BV, v. 86, p. 597-621, jan 2018. Citations on pages 35 and 37.

HAM, A. M. Integrated scheduling of m-truck, m-drone, and m-depot constrained by time-window, drop-pickup, and m-visit using constraint programming. Transportation Research Part C: Emerging Technologies, Elsevier BV, v. 91, p. 1-14, jun 2018. Citations on pages 35 and 37.

HONG, I.; KUBY, M.; MURRAY, A. T. A range-restricted recharging station coverage model for drone delivery service planning. Transportation Research Part C: Emerging Technologies, Elsevier BV, v. 90, p. 198-212, may 2018. Citation on page 27.

INTEL. Intel Drones at The Olympic Games. 2018. Available: <https://www.intel. com/content/www/us/en/sports/olympic-games/drones.html>. Citation on page 25. 
KüCKELHAUS, M.; NIEZGODA, D.; ENDRIß, S. resreport, Unmanned Aerial Vehicles In Logistics: A dhl perspective on implications and use cases for the logistics industry. 2014. Available: <http://www.dhl.com/content/dam/downloads/g0/about_us/ logistics_insights/dhl_trend_report_uav.pdf $>$. Citations on pages 27 and 41.

KUHLMANN, D. DHL Parcelcopter 3.0. 2016. Available: <http://www.dpdhl.com/ en/media_relations/specials/parcelcopter.html>. Citation on page 25.

LAPORTE, G. Fifty years of vehicle routing. Transportation Science, Institute for Operations Research and the Management Sciences (INFORMS), v. 43, n. 4, p. 408-416, nov 2009. Citation on page 30.

LENSTRA, J. K.; KAN, A. H. G. R. Complexity of vehicle routing and scheduling problems. Networks, Wiley-Blackwell, v. 11, n. 2, p. 221-227, 1981. Citation on page 29.

LIN, C.; CHOY, K.; HO, G.; CHUNG, S.; LAM, H. Survey of green vehicle routing problem: Past and future trends. Expert Systems with Applications, Elsevier BV, v. 41, n. 4, p. 1118-1138, mar 2014. Citation on page 31.

LITHIA, F. UPS Tests Residential Delivery Via Drone Launched From atop Package Car: Autonomous drone delivery system. 2017. Available: <https://www.pressroom.ups.com/pressroom/ContentDetailsViewer.page? ConceptType $=$ PressReleases\&id $=1487687844847-162>$. Citations on pages 25 and 28.

LUO, Z.; LIU, Z.; SHI, J. A two-echelon cooperated routing problem for a ground vehicle and its carried unmanned aerial vehicle. Sensors, MDPI AG, v. 17, n. 5, p. 1144, may 2017. Citations on pages 34 and 37.

MATHEW, N.; SMITH, S. L.; WASLANDER, S. L. Planning paths for package delivery in heterogeneous multirobot teams. IEEE Transactions on Automation Science and Engineering, Institute of Electrical and Electronics Engineers (IEEE), v. 12, n. 4, p. 1298-1308, oct 2015. Citations on pages 36 and 37.

MEIER, P. Humanitarian in the sky: drones for disaster response. 2018. Available: <https://www.virgin.com/virgin-unite/business-innovation/ humanitarian-sky-drones-disaster-response $>$. Citation on page 25.

MEOLA, A. Drone market shows positive outlook with strong industry growth and trends. 2017. Available: <http://www.businessinsider.com/ drone-industry-analysis-market-trends-growth-forecasts-2017-7>. Citation on page 25.

MURRAY, C. C.; CHU, A. G. The flying sidekick traveling salesman problem: Optimization of drone-assisted parcel delivery. Transportation Research Part C: Emerging Technologies, Elsevier BV, v. 54, p. 86-109, may 2015. Citations on pages 11, 13, 28, $31,32,33,35,37,43,50,85$, and 93 .

NETWORKInG; OPTIMIZATION, E. Vehicle Routing Problem. 2013. Available: $<$ http://neo.lcc.uma.es/vrp/vehicle-routing-problem/>. Citation on page 30.

O'KANE, S. Hamburgers and beer are about to start flying through the skies of Reykjavík, Iceland. 2017. Available: <https://www.theverge.com/2017/8/23/ 16185112/drone-delivery-iceland-flytrex-aha-food $>$. Citations on pages 26 and 113. 
OTHMAN, M. S. bin; SHURBEVSKI, A.; KARUNO, Y.; NAGAMOCHI, H. Routing of carrier-vehicle systems with dedicated last-stretch delivery vehicle and fixed carrier route. Journal of Information Processing, Information Processing Society of Japan, v. 25, n. 0, p. 655-666, 2017. Citations on pages 36 and 37.

POIKONEN, S.; WANG, X.; GOLDEN, B. The vehicle routing problem with drones: Extended models and connections. Networks, Wiley-Blackwell, v. 70, n. 1, p. 34-43, jun 2017. Citations on pages 32 and 37.

PONZA, A. Optimization of Drone-assisted Parcel Delivery. Master's Thesis (Master's Thesis), 2016. Citations on pages 36 and 37.

PRINS, C. A simple and effective evolutionary algorithm for the vehicle routing problem. Computers \& Operations Research, Elsevier BV, v. 31, n. 12, p. 1985-2002, oct 2004. Citations on pages 51, 53, and 58 .

PUGLIESE, L. D. P.; GUERRIERO, F. Last-mile deliveries by using drones and classical vehicles. In: Springer Proceedings in Mathematics \& Statistics. [S.l.]: Springer International Publishing, 2017. p. 557-565. Citations on pages 33, 37, and 86.

PULVER, A.; WEI, R. Optimizing the spatial location of medical drones. Applied Geography, Elsevier BV, v. 90, p. 9-16, jan 2018. Citation on page 25.

PUREZA, V.; MORABITO, R.; REIMANN, M. Vehicle routing with multiple deliverymen: Modeling and heuristic approaches for the VRPTW. European Journal of Operational Research, Elsevier BV, v. 218, n. 3, p. 636-647, may 2012. Citation on page 30 .

SCHERMER, D.; MOEINI, M.; WENDT, O. Algorithms for solving the vehicle routing problem with drones. In: Intelligent Information and Database Systems. [S.1.]: Springer International Publishing, 2018. p. 352-361. Citations on pages 33 and 37.

SCHOLZ, N. Pizza-by-drone a reality with world-first customer deliveries in New Zealand. 2016. Available: <https://www.dominos.com.au/inside-dominos/media/ november-2016-pizza-by-drone-a-reality-with-world-first-customer-deliveries-in-new-zealand $>$. Citation on page 25.

SEILER, B. University of Maryland's Schools of Medicine and Engineering First to Use Unmanned Aircraft to Successfully Deliver Kidney for Transplant at University of Maryland Medical Center. 2019. Available: <https://www.umms.org/ ummc/news/2019/pioneering-breakthrough-unmanned-aircraft $>$. Citation on page 25 .

SHAW, S. 7-Eleven Teams with Flirtey for First Ever FAA-Approved Drone Delivery to Customer's Home. 2016. Available: <http://corp.7-eleven.com/corp-press-releases/ 07-22-2016-7-eleven-teams-with-flirtey-for-first-ever-faa-approved-drone-delivery-to-customer-s-home $>$. Citation on page 25.

SIMMONS, D. Rwanda begins Zipline commercial drone deliveries. 2016. Available: <http://www.bbc.com/news/technology-37646474>. Citation on page 25. 
SOLOMON, M. M. Algorithms for the vehicle routing and scheduling problems with time window constraints. Operations Research, Institute for Operations Research and the Management Sciences (INFORMS), v. 35, n. 2, p. 254-265, apr 1987. Citations on pages $15,67,81,82,83$, and 85 .

STOLAROFF, J.; SAMARAS, C. Delivering packages with drones may benefit the environment. 2018. Available: <https://www.weforum.org/agenda/2018/02/ delivering-packages-with-drones-might-be-good-for-the-environment $>$. Citation on page 26.

ULMER, M. W.; THOMAS, B. W. Same-day delivery with heterogeneous fleets of drones and vehicles. Networks, Wiley Online Library, v. 72, n. 4, p. 475-505, 2018. Citations on pages 36 and 37.

VIDAL, T.; CRAINIC, T. G.; GENDREAU, M.; LAHRICHI, N.; REI, W. A hybrid genetic algorithm for multidepot and periodic vehicle routing problems. Operations Research, Institute for Operations Research and the Management Sciences (INFORMS), v. 60, n. 3, p. 611-624, jun 2012. Citation on page 53.

VIDAL, T.; CRAINIC, T. G.; GENDREAU, M.; PRINS, C. A unified solution framework for multi-attribute vehicle routing problems. European Journal of Operational Research, Elsevier BV, v. 234, n. 3, p. 658-673, may 2014. Citations on pages 51 and 85.

WANG, X.; POIKONEN, S.; GOLDEN, B. The vehicle routing problem with drones: several worst-case results. Optimization Letters, Springer Nature, v. 11, n. 4, p. 679-697, apr 2016. Citations on pages 28, 32, 33, and 37.

WREN, A.; HOLLIDAY, A. Computer scheduling of vehicles from one or more depots to a number of delivery points. Journal of the Operational Research Society, Informa UK Limited, v. 23, n. 3, p. 333-344, sep 1972. Citation on page 55.

XCOMPANY. Project Wing. 2017. Available: <https://x.company/wing/>. Citation on page 25 . 


(MURRAY; CHU, 2015).

$$
\begin{aligned}
& \operatorname{Min} t_{c+1} \\
& \text { s.t. } \sum_{\substack{i \in N_{0} \\
i \neq j}} x_{i j}+\sum_{\substack{i \in N_{0} \\
i \neq j}} \sum_{\substack{k \in N_{+} \\
(i, j, k) \in P}} y_{i j k}=1 \quad \forall j \in C \\
& \sum_{j \in N_{+}} x_{0 j}=1 \\
& \sum_{i \in N_{0}} x_{i, c+1}=1 \\
& \sum_{i \in N_{0}} x_{i, c+1}=1 \\
& u_{i}-u_{j}+1 \leq(c+2)\left(1-x_{i j}\right) \quad \forall i \in C, j \in\left\{N_{+}: j \neq i\right\} \\
& \sum_{\substack{i \in N_{0} \\
i \neq j}} x_{i j}=\sum_{\substack{k \in N_{+} \\
k \neq j}} x_{k j} \quad \forall j \in C \\
& \sum_{\substack{j \in C \\
j \neq i}} \sum_{\substack{k \in N_{+} \\
(i, j, k) \in P}} y_{i j k} \leq 1 \quad \forall i \in N_{0}
\end{aligned}
$$




$$
\begin{aligned}
& \sum_{\substack{i \in N_{0} \\
i \neq k}} \sum_{\substack{j \in C \\
i, j, k) \in P}} y_{i j k} \leq 1 \quad \forall k \in N_{+} \\
& 2 y_{i j k} \leq \sum_{\substack{h \in N_{0} \\
h \neq i}} x_{h i}+\sum_{\substack{l \in C \\
l \neq k}} x_{l k} \quad \forall i \in C, j \in\{C: j \neq i\}, k \in\left\{N_{+}:(i, j, k) \in P\right\} \\
& y_{0 j k} \leq \sum_{\substack{h \in N_{0} \\
h \neq k}} x_{h k} \quad \forall j \in C, k \in\left\{N_{+}:(0, j, k) \in P\right\} \\
& u_{k}-u_{i} \geq 1-(c+2)\left(1-\sum_{\substack{j \in C \\
(i, j, k) \in P}} y_{i j k}\right) \quad \forall i \in C, k \in\left\{N_{+}: k \neq i\right\} \\
& t_{i}^{\prime} \geq t_{i}-M\left(1-\sum_{\substack{j \in C \\
j \neq i}} \sum_{\substack{k \in N_{+} \\
(i, j, k) \in P}} y_{i j k}\right) \quad \forall i \in C \\
& t_{i}^{\prime} \leq t_{i}+M\left(1-\sum_{\substack{j \in C \\
j \neq i}} \sum_{\substack{k \in N_{+} \\
(i, j, k) \in P}} y_{i j k}\right) \quad \forall i \in C \\
& t_{k}^{\prime} \geq t_{k}-M\left(1-\sum_{\substack{i \in N_{0} \\
i \neq k}} \sum_{\substack{\left.j \in j_{j}, j, k\right) \in P \\
y_{i j k}}} y_{i j} \quad \forall k \in N_{+}\right. \\
& t_{k}^{\prime} \leq t_{k}+M\left(1-\sum_{\substack{i \in N_{0} \\
i \neq k}} \sum_{\substack{j \in C \\
i, j, k) \in P}} y_{i j k}\right) \quad \forall k \in N_{+} \\
& \left.\left.t_{k} \geq t_{h}+\tau_{h k}+S_{L}\left(\sum_{\substack{l \in C \\
l \neq k}} \sum_{\substack{m \in N_{+} \\
(k, l, m) \in P}} y_{k l m}\right)\right)+S_{R}\left(\sum_{\substack{i \in N_{0} \\
i \neq k}} \sum_{\substack{j \in C \\
i, j, k) \in P}} y_{i j k}\right)\right)-M\left(1-X_{h k}\right) \quad \forall h \in N_{0}, k \in\left\{N_{+}: k \neq h\right\} \\
& t_{j}^{\prime} \geq t_{i}^{\prime}+\tau_{i j}^{\prime}-M\left(1-\sum_{\substack{k \in N_{+} \\
(i, j, k) \in P}} y_{i j k}\right) \quad \forall j \in C^{\prime}, i \in\left\{N_{0}: i \neq j\right\}
\end{aligned}
$$




$$
\begin{gathered}
t_{k}^{\prime} \geq t_{j}^{\prime}+\tau_{j k}^{\prime}+S_{R}-M\left(1-\sum_{\substack{i \in N_{0} \\
(i, j, k) \in P}} y_{i j k}\right) \quad \forall j \in C^{\prime}, k \in\left\{N_{+}: k \neq j\right\} \\
t_{k}^{\prime}-\left(t_{j}^{\prime}-\tau_{i j}^{\prime}\right) \leq e+M\left(1-y_{i j k}\right) \quad \forall k \in N_{+}, j \in\{C: j \neq k\}, i \in\left\{N_{0}:(i, j, k) \in P\right\} \\
u_{i}-u_{j} \geq 1-(c+2) p_{i j} \quad \forall i \in C, j \in\{C: j \neq i\} \\
u_{i}-u_{j} \leq-1+(c+2)\left(1-p_{i j}\right) \quad \forall i \in C, j \in\{C: j \neq i\} \\
p_{i j}+p_{j i}=1 \quad \forall i \in C, j \in\{C: j \neq i\}
\end{gathered}
$$$$
t_{l}^{\prime} \geq t_{k}^{\prime}-M\left(3-\sum_{\substack{j \in C \\(i, j, k) \in P \\ j \neq l}} y_{i j k}-\sum_{\substack{m \in C \\ m \neq i \\ m \neq k \\ m \neq k}} \sum_{\substack{\left.n \in N_{+} \\ m \neq n, m\right) \in P \\ m \neq i \\ n \neq k}} y_{l m n}-p_{i l}\right) \quad \forall i \in N_{0}, k \in\left\{N_{+}: k \neq i\right\}, l \in\{C: l \neq i, l \neq k\}
$$

$$
t_{0}=0
$$$$
t_{0}^{\prime}=0
$$

$$
p_{0 j}=1 \quad \forall j \in C
$$$$
x_{i j} \in\{0,1\} \quad \forall i \in N_{0}, j \in\left\{N_{+}: j \neq i\right\}
$$

$y_{i j k} \in\{0,1\} \quad \forall i \in N_{0}, j \in\{C: j \neq i\}, k \in\left\{N_{+}:(i, j, k) \in P\right\}$

$$
1 \leq u_{i} \leq c+2 \quad \forall i \in N_{+}
$$




$$
\begin{gathered}
t_{i} \geq 0 \quad \forall i \in N \\
t_{i}^{\prime} \geq 0 \quad \forall i \in N \\
p_{i j} \in\{0,1\} \quad \forall i \in N_{0}, j \in\{C: j \neq i\}
\end{gathered}
$$




\section{ADDITIONAL RESULTS AND TABLES}

Here we present additional results according to the specifications in Chapter 5.

\section{B.1 MILP + Sweep (cont.)}

The first set of results are using the Sweep algorithm as a start solution. We test the model with the other two drone eligible weight parameter values: $25 \%$ and $75 \%$. The main additional results observed are the lower gaps in the $25 \%$ family, as well as the greater gaps in the $\mathbf{7 5 \%}$, as mentioned in Chapter 5 .

Table 21 - Results for the class of instances C, with 100\% drone speed and 25\% drone eligible weight, using the Sweep as a starting solution.

\begin{tabular}{l|c|c|c|c|c|c|c}
\hline Instance & $\begin{array}{c}\text { Objective } \\
\text { Function }\end{array}$ & GAP & Dual & Exec. Time & Nodes & Variables & Constraints \\
\hline $\mathrm{c} 25101$ & 181.50 & 0.00 & 181.50 & 21.03 & 2604 & 41405 & 501301 \\
\hline $\mathrm{c} 25102$ & 637.95 & 0.82 & 115.93 & 3600.61 & 3 & 54705 & 518251 \\
\hline $\mathrm{c} 25103$ & 502.13 & 0.79 & 103.26 & 3601.61 & 1 & 58805 & 524751 \\
\hline $\mathrm{c} 25104$ & 389.05 & 0.73 & 103.26 & 3601.61 & 2 & 62855 & 529676 \\
\hline $\mathrm{c} 25105$ & 177.94 & 0.00 & 177.94 & 656.16 & 72798 & 44955 & 505526 \\
\hline $\mathrm{c} 25106$ & 177.94 & 0.00 & 177.94 & 27.91 & 885 & 42455 & 502451 \\
\hline $\mathrm{c} 25107$ & 323.87 & 0.51 & 157.51 & 3622.14 & 34469 & 46030 & 507101 \\
\hline $\mathrm{c} 25108$ & 469.70 & 0.89 & 52.13 & 3845.14 & 10422 & 50455 & 512926 \\
\hline $\mathrm{c} 25109$ & 436.62 & 0.89 & 48.90 & 3601.28 & 5 & 53855 & 517626 \\
\hline $\mathrm{c} 25201$ & 209.89 & 0.00 & 209.89 & 59.43 & 16940 & 41455 & 501951 \\
\hline $\mathrm{c} 25202$ & 589.37 & 0.79 & 125.16 & 3601.83 & 15156 & 49155 & 513301 \\
\hline $\mathrm{c} 25204$ & 550.19 & 0.81 & 104.36 & 3602.91 & 72 & 55530 & 521851 \\
\hline $\mathrm{c} 25205$ & 547.24 & 0.77 & 104.36 & 3603.72 & 173 & 57180 & 524451 \\
\hline $\mathrm{c} 25206$ & 438.65 & 0.76 & 133.08 & 3601.16 & 3739 & 45680 & 507151 \\
\hline $\mathrm{c} 25207$ & 445.57 & 0.76 & 110.89 & 3601.60 & 973 & 47280 & 509376 \\
\hline $\mathrm{c} 25208$ & 398.32 & 0.74 & 104.42 & 3602.74 & 57 & 50155 & 513451 \\
\hline
\end{tabular}


Table 22 - Results for the class of instances C, with 100\% drone speed and 75\% drone eligible weight, using the Sweep as a starting solution.

\begin{tabular}{l|c|c|c|c|c|c|c}
\hline Instance & $\begin{array}{c}\text { Objective } \\
\text { Function }\end{array}$ & GAP & Dual & Exec. Time & Nodes & Variables & Constraints \\
\hline $\mathrm{c} 25101$ & 181.5 & 0.0 & 181.5 & 130.3 & 14793 & 54305 & 526501 \\
\hline $\mathrm{c} 25102$ & 637.9 & 0.9 & 46.1 & 3600.7 & 5 & 73005 & 548826 \\
\hline $\mathrm{c} 25103$ & 502.1 & 1.0 & 15.1 & 3602.2 & 1 & 78930 & 557126 \\
\hline $\mathrm{c} 25104$ & 389.0 & 1.0 & 14.5 & 3600.1 & 0 & 85055 & 564126 \\
\hline $\mathrm{c} 25105$ & 202.8 & 0.4 & 114.8 & 3600.7 & 69690 & 60480 & 533376 \\
\hline $\mathrm{c} 25106$ & 177.9 & 0.0 & 177.9 & 395.6 & 29453 & 55855 & 528151 \\
\hline $\mathrm{c} 25107$ & 661.2 & 0.9 & 86.6 & 3600.6 & 2 & 61555 & 534951 \\
\hline $\mathrm{c} 25108$ & 469.7 & 1.0 & 17.3 & 3601.1 & 1 & 67480 & 542226 \\
\hline $\mathrm{c} 25109$ & 436.6 & 1.0 & 15.3 & 3602.4 & 1 & 72955 & 548976 \\
\hline $\mathrm{c} 25201$ & 201.9 & 0.0 & 201.9 & 82.7 & 12808 & 57630 & 530426 \\
\hline $\mathrm{c} 25202$ & 589.4 & 0.9 & 80.5 & 3601.3 & 41 & 68355 & 544776 \\
\hline $\mathrm{c} 25203$ & 550.2 & 0.9 & 39.1 & 3602.6 & 1 & 76780 & 555351 \\
\hline $\mathrm{c} 25204$ & 461.2 & 0.9 & 33.4 & 3600.4 & 0 & 80705 & 560226 \\
\hline $\mathrm{c} 25205$ & 547.2 & 0.8 & 88.8 & 3600.8 & 421 & 64005 & 537751 \\
\hline $\mathrm{c} 25206$ & 438.7 & 0.9 & 39.2 & 3609.6 & 93 & 66655 & 541001 \\
\hline $\mathrm{c} 25207$ & 445.6 & 0.9 & 49.6 & 3603.0 & 1 & 71730 & 547276 \\
\hline $\mathrm{c} 25208$ & 398.3 & 0.9 & 36.4 & 3600.8 & 0 & 69480 & 544401 \\
\hline
\end{tabular}

Table 23 - Results for the class of instances R, with 100\% drone speed and 25\% drone eligible weight, using the Sweep as a starting solution.

\begin{tabular}{l|c|c|c|c|c|c|c}
\hline Instance & $\begin{array}{c}\text { Objective } \\
\text { Function }\end{array}$ & GAP & Dual & Exec. Time & Nodes & Variables & Constraints \\
\hline $\mathrm{r} 25101$ & 595.04 & 0.00 & 595.04 & 12.73 & 8596 & 27030 & 476776 \\
\hline $\mathrm{r} 25102$ & 773.97 & 0.58 & 323.58 & 3600.55 & 10471 & 35055 & 489651 \\
\hline $\mathrm{r} 25103$ & 678.39 & 0.65 & 237.06 & 3601.40 & 245 & 38005 & 495401 \\
\hline $\mathrm{r} 25104$ & 582.78 & 0.62 & 221.34 & 3601.63 & 531 & 40380 & 499151 \\
\hline $\mathrm{r} 25105$ & 469.19 & 0.16 & 396.02 & 3607.27 & 66410 & 30530 & 482226 \\
\hline $\mathrm{r} 25106$ & 656.17 & 0.61 & 255.88 & 3606.60 & 7600 & 36830 & 492676 \\
\hline $\mathrm{r} 25107$ & 617.44 & 0.62 & 237.06 & 3601.19 & 39 & 39030 & 497076 \\
\hline $\mathrm{r} 25108$ & 594.70 & 0.63 & 221.34 & 3601.72 & 221 & 40880 & 500126 \\
\hline $\mathrm{r} 25109$ & 689.29 & 0.60 & 272.29 & 3604.93 & 13804 & 33755 & 488201 \\
\hline $\mathrm{r} 25110$ & 563.54 & 0.58 & 239.24 & 3601.59 & 958 & 38405 & 495826 \\
\hline $\mathrm{r} 25111$ & 653.14 & 0.64 & 237.06 & 3601.64 & 81 & 38355 & 495801 \\
\hline $\mathrm{r} 25112$ & 522.93 & 0.58 & 221.34 & 3601.40 & 1414 & 42105 & 502576 \\
\hline $\mathrm{r} 25201$ & 435.36 & 0.00 & 435.33 & 1851.36 & 142571 & 32980 & 487126 \\
\hline $\mathrm{r} 25202$ & 635.44 & 0.62 & 243.94 & 3600.88 & 1018 & 38255 & 495526 \\
\hline $\mathrm{r} 25203$ & 612.00 & 0.61 & 237.06 & 3601.36 & 547 & 40205 & 499426 \\
\hline $\mathrm{r} 25204$ & 536.87 & 0.59 & 221.34 & 3601.66 & 1585 & 41630 & 501626 \\
\hline $\mathrm{r} 25205$ & 565.29 & 0.49 & 286.09 & 3608.67 & 14205 & 35830 & 492276 \\
\hline $\mathrm{r} 25206$ & 579.00 & 0.61 & 226.07 & 3601.26 & 1261 & 39455 & 497901 \\
\hline $\mathrm{r} 25207$ & 548.74 & 0.59 & 226.07 & 3615.47 & 2204 & 40555 & 500101 \\
\hline $\mathrm{r} 25208$ & 533.49 & 0.59 & 221.34 & 3601.79 & 1560 & 41730 & 501826 \\
\hline $\mathrm{r} 25209$ & 512.67 & 0.53 & 243.06 & 3601.78 & 110 & 38555 & 496476 \\
\hline $\mathrm{r} 25210$ & 605.60 & 0.56 & 265.94 & 3607.66 & 3934 & 39105 & 497401 \\
\hline $\mathrm{r} 25211$ & 475.78 & 0.53 & 221.34 & 3601.91 & 1223 & 42130 & 502626 \\
\hline
\end{tabular}


Table 24 - Results for the class of instances R, with 100\% drone speed and 75\% drone eligible weight, using the Sweep as a starting solution.

\begin{tabular}{l|c|c|c|c|c|c|c}
\hline Instance & $\begin{array}{c}\text { Objective } \\
\text { Function }\end{array}$ & GAP & Dual & Exec. Time & Nodes & Variables & Constraints \\
\hline $\mathrm{r} 25101$ & 478.69 & 0.00 & 478.69 & 39.87 & 10951 & 34305 & 498876 \\
\hline $\mathrm{r} 25102$ & 773.97 & 0.81 & 147.20 & 3600.47 & 8 & 45730 & 515101 \\
\hline $\mathrm{r} 25103$ & 678.39 & 0.82 & 119.12 & 3601.24 & 0 & 50905 & 523051 \\
\hline $\mathrm{r} 25104$ & 582.78 & 0.81 & 112.49 & 3602.33 & 1 & 54330 & 527851 \\
\hline $\mathrm{r} 25105$ & 480.53 & 0.45 & 264.30 & 3601.03 & 145729 & 40005 & 506501 \\
\hline $\mathrm{r} 25106$ & 782.37 & 0.84 & 127.74 & 3601.57 & 0 & 48730 & 519326 \\
\hline $\mathrm{r} 25107$ & 617.44 & 0.81 & 119.00 & 3601.97 & 1 & 52105 & 524901 \\
\hline $\mathrm{r} 25108$ & 594.70 & 0.81 & 112.49 & 3601.13 & 0 & 54980 & 528976 \\
\hline $\mathrm{r} 25109$ & 689.29 & 0.77 & 158.03 & 3601.46 & 2 & 44755 & 514026 \\
\hline $\mathrm{r} 25110$ & 563.54 & 0.79 & 117.06 & 3601.43 & 0 & 51880 & 524101 \\
\hline $\mathrm{r} 25111$ & 653.14 & 0.82 & 119.04 & 3602.18 & 1 & 51030 & 523226 \\
\hline $\mathrm{r} 25112$ & 522.93 & 0.79 & 107.97 & 3602.39 & 1 & 56430 & 531651 \\
\hline $\mathrm{r} 25201$ & 523.06 & 0.48 & 272.37 & 3600.41 & 23444 & 42430 & 511376 \\
\hline $\mathrm{r} 25202$ & 635.44 & 0.79 & 133.61 & 3601.25 & 1 & 50255 & 522276 \\
\hline $\mathrm{r} 25203$ & 612.00 & 0.80 & 119.50 & 3601.82 & 1 & 53355 & 527326 \\
\hline $\mathrm{r} 25204$ & 536.87 & 0.79 & 112.49 & 3603.04 & 1 & 55755 & 530501 \\
\hline $\mathrm{r} 25205$ & 565.43 & 0.68 & 178.51 & 3611.17 & 1754 & 47430 & 518676 \\
\hline $\mathrm{r} 25206$ & 579.00 & 0.80 & 114.95 & 3602.05 & 2 & 52280 & 525476 \\
\hline $\mathrm{r} 25207$ & 548.74 & 0.79 & 113.33 & 3602.01 & 3 & 53980 & 528276 \\
\hline $\mathrm{r} 25208$ & 533.49 & 0.79 & 112.40 & 3602.78 & 1 & 55930 & 530776 \\
\hline $\mathrm{r} 25209$ & 512.67 & 0.76 & 123.53 & 3601.05 & 2 & 51005 & 523701 \\
\hline $\mathrm{r} 25210$ & 605.60 & 0.75 & 151.68 & 3601.19 & 2 & 51605 & 524651 \\
\hline $\mathrm{r} 25211$ & 475.78 & 0.77 & 107.97 & 3601.34 & 0 & 56455 & 531701 \\
\hline
\end{tabular}

Table 25 - Results for the class of instances RC, with 100\% drone speed and 25\% drone eligible weight, using the Sweep as a starting solution.

\begin{tabular}{l|c|c|c|c|c|c|c}
\hline Instance & $\begin{array}{c}\text { Objective } \\
\text { Function }\end{array}$ & GAP & Dual & Exec. Time & Nodes & Variables & Constraints \\
\hline $\mathrm{rc} 25101$ & 394.08 & 0.40 & 238.13 & 3600.41 & 60916 & 33255 & 485676 \\
\hline $\mathrm{rc} 25102$ & 764.73 & 0.86 & 103.89 & 3607.86 & 1231 & 39355 & 495901 \\
\hline $\mathrm{rc} 25103$ & 622.29 & 0.86 & 84.70 & 3602.25 & 4317 & 44530 & 503726 \\
\hline $\mathrm{rc} 25104$ & 573.94 & 0.86 & 82.31 & 3602.77 & 609 & 45905 & 506251 \\
\hline $\mathrm{rc} 25105$ & 875.21 & 0.80 & 176.33 & 3698.49 & 8167 & 38155 & 493001 \\
\hline $\mathrm{rc} 25106$ & 695.34 & 0.84 & 113.08 & 3601.65 & 1071 & 38505 & 493776 \\
\hline $\mathrm{rc} 25107$ & 747.24 & 0.90 & 78.31 & 3602.49 & 659 & 43955 & 502376 \\
\hline $\mathrm{rc} 25108$ & 587.71 & 0.87 & 78.31 & 3603.03 & 241 & 46780 & 507776 \\
\hline $\mathrm{rc} 25201$ & 336.95 & 0.32 & 228.47 & 3600.58 & 245369 & 35580 & 491151 \\
\hline $\mathrm{rc} 25202$ & 794.62 & 0.88 & 96.88 & 3609.65 & 4055 & 40780 & 499426 \\
\hline $\mathrm{rc} 25203$ & 738.73 & 0.89 & 84.70 & 3602.12 & 634 & 45830 & 506326 \\
\hline $\mathrm{rc} 25204$ & 519.67 & 0.84 & 82.31 & 3602.68 & 207 & 46830 & 508101 \\
\hline $\mathrm{rc25205}$ & 738.90 & 0.76 & 176.63 & 3601.30 & 1020 & 40755 & 498426 \\
\hline $\mathrm{rc} 25206$ & 583.21 & 0.81 & 113.08 & 3601.20 & 593 & 40930 & 498826 \\
\hline $\mathrm{rc} 25207$ & 539.85 & 0.84 & 87.88 & 3602.15 & 471 & 44455 & 504051 \\
\hline $\mathrm{rc} 25208$ & 454.11 & 0.83 & 78.31 & 3603.43 & 351 & 47705 & 509551 \\
\hline
\end{tabular}


Table 26 - Results for the class of instances RC, with 100\% drone speed and 75\% drone eligible weight, using the Sweep as a starting solution.

\begin{tabular}{l|c|c|c|c|c|c|c}
\hline Instance & $\begin{array}{c}\text { Objective } \\
\text { Function }\end{array}$ & GAP & Dual & Exec. Time & Nodes & Variables & Constraints \\
\hline $\mathrm{rc} 25101$ & 869.38 & 0.92 & 65.97 & 3601.17 & 187 & 54030 & 523651 \\
\hline $\mathrm{rc} 25102$ & 764.73 & 0.95 & 41.00 & 3601.50 & 1 & 67580 & 541301 \\
\hline $\mathrm{rc} 25103$ & 622.29 & 0.95 & 31.01 & 3600.14 & 0 & 75005 & 551351 \\
\hline $\mathrm{rc} 25104$ & 573.94 & 0.96 & 24.95 & 3600.27 & 0 & 79555 & 557051 \\
\hline $\mathrm{rc} 25105$ & 875.21 & 0.96 & 38.58 & 3601.80 & 0 & 64605 & 536601 \\
\hline $\mathrm{rc} 25106$ & 695.34 & 0.95 & 31.98 & 3614.04 & 0 & 65805 & 538226 \\
\hline $\mathrm{rc} 25107$ & 747.24 & 0.97 & 24.88 & 3600.40 & 0 & 77205 & 552776 \\
\hline $\mathrm{rc} 25108$ & 587.71 & 0.96 & 24.84 & 3601.33 & 0 & 81555 & 559701 \\
\hline $\mathrm{rc} 25201$ & 804.39 & 0.84 & 130.67 & 3600.78 & 568 & 57630 & 530376 \\
\hline $\mathrm{rc} 25202$ & 794.62 & 0.95 & 42.14 & 3601.04 & 4 & 69355 & 545151 \\
\hline $\mathrm{rc} 25203$ & 738.73 & 0.96 & 31.02 & 3601.09 & 0 & 76730 & 554376 \\
\hline $\mathrm{rc} 25205$ & 519.67 & 0.95 & 24.95 & 3600.23 & 0 & 80505 & 558926 \\
\hline $\mathrm{rc} 25206$ & 583.90 & 0.92 & 56.08 & 3600.65 & 0 & 67080 & 541901 \\
\hline $\mathrm{rc} 25207$ & 539.85 & 0.94 & 35.86 & 3601.52 & 1 & 67680 & 542726 \\
\hline $\mathrm{rc} 25208$ & 454.11 & 0.95 & 25.04 & 3601.70 & 0 & 75780 & 552551 \\
\hline
\end{tabular}

Table 27 - Results for the class of instances C, with 50\% drone speed and 25\% drone eligible weight, using the HGA as a starting solution.

\begin{tabular}{l|c|c|c|c|c|c|c}
\hline Instance & $\begin{array}{c}\text { Objective } \\
\text { Function }\end{array}$ & GAP & Dual & Exec. Time & Nodes & Variables & Constraints \\
\hline $\mathrm{c} 25101$ & 191.81 & 0.00 & 191.81 & 5.10 & 0 & 30655 & 489777 \\
\hline $\mathrm{c} 25102$ & 190.74 & 0.22 & 148.77 & 3600.37 & 63 & 38405 & 501152 \\
\hline $\mathrm{c} 25103$ & 190.74 & 0.42 & 110.61 & 3601.30 & 283 & 41530 & 506702 \\
\hline $\mathrm{c} 25104$ & 187.45 & 0.45 & 103.41 & 3600.88 & 5 & 43255 & 509302 \\
\hline $\mathrm{c} 25105$ & 191.81 & 0.00 & 191.81 & 8.43 & 0 & 32705 & 492502 \\
\hline $\mathrm{c} 25106$ & 191.81 & 0.00 & 191.81 & 5.65 & 0 & 31255 & 490477 \\
\hline $\mathrm{c} 25107$ & 191.81 & 0.00 & 191.80 & 747.67 & 45860 & 33530 & 493827 \\
\hline $\mathrm{c} 25108$ & 191.81 & 0.52 & 92.59 & 3600.89 & 4751 & 36130 & 497827 \\
\hline $\mathrm{c} 25109$ & 191.81 & 0.72 & 53.37 & 3601.32 & 106 & 38005 & 500977 \\
\hline $\mathrm{c} 25201$ & 214.88 & 0.00 & 214.88 & 4.87 & 0 & 26680 & 486327 \\
\hline $\mathrm{c} 25202$ & 214.88 & 0.31 & 149.17 & 3600.49 & 39948 & 30530 & 493827 \\
\hline $\mathrm{c} 25203$ & 213.19 & 0.36 & 135.91 & 3617.12 & 2394 & 33280 & 498827 \\
\hline $\mathrm{c} 25204$ & 218.64 & 0.41 & 128.67 & 3600.70 & 5887 & 34230 & 500727 \\
\hline $\mathrm{c} 25205$ & 214.88 & 0.26 & 159.54 & 3600.38 & 248684 & 27930 & 488627 \\
\hline $\mathrm{c} 25206$ & 214.88 & 0.36 & 136.80 & 3632.69 & 22728 & 28705 & 490027 \\
\hline $\mathrm{c} 25207$ & 214.67 & 0.26 & 158.05 & 3600.81 & 12520 & 30105 & 492627 \\
\hline $\mathrm{c} 25208$ & 214.88 & 0.39 & 130.72 & 3606.91 & 9641 & 29480 & 491377 \\
\hline
\end{tabular}

\section{B.2 MILP + HGA (cont.)}

The second set of results are using the HGA algorithm as a start solution. Since this is our main contribution, we test the model with all other parameter values: drone eligible weight of $25 \%, 50 \%$ and $75 \%$; drone speed of $50 \%$, the default of $100 \%$ and the faster $150 \%$. Resulting in a combination of 9 combinations per instance class (the default parameter results are in the Chapter 5). The results in this section corroborate the increase in drone eligible customers being more detrimental to the model's solvability . 
Table 28 - Results for the class of instances C, with 50\% drone speed and 50\% drone eligible weight, using the HGA as a starting solution.

\begin{tabular}{l|c|c|c|c|c|c|c}
\hline Instance & $\begin{array}{c}\text { Objective } \\
\text { Function }\end{array}$ & GAP & Dual & Exec. Time & Nodes & Variables & Constraints \\
\hline $\mathrm{c} 25101$ & 191.81 & 0.00 & 191.81 & 6.53 & 0 & 34455 & 503527 \\
\hline $\mathrm{c} 25102$ & 190.74 & 0.26 & 141.34 & 3600.45 & 4 & 44780 & 517477 \\
\hline $\mathrm{c} 25103$ & 190.74 & 0.53 & 89.29 & 3600.45 & 0 & 48105 & 523177 \\
\hline $\mathrm{c} 25104$ & 187.45 & 0.57 & 80.27 & 3600.69 & 0 & 50580 & 526527 \\
\hline $\mathrm{c} 25105$ & 191.81 & 0.00 & 191.81 & 19.08 & 0 & 37380 & 507127 \\
\hline $\mathrm{c} 25106$ & 191.81 & 0.00 & 191.81 & 7.47 & 0 & 35280 & 504452 \\
\hline $\mathrm{c} 25107$ & 191.81 & 0.36 & 122.19 & 3600.87 & 3885 & 38205 & 508452 \\
\hline $\mathrm{c} 25108$ & 191.81 & 0.81 & 35.60 & 3601.54 & 2214 & 41580 & 513202 \\
\hline $\mathrm{c} 25109$ & 191.81 & 0.85 & 28.59 & 3601.18 & 5 & 44180 & 517077 \\
\hline $\mathrm{c} 25201$ & 214.88 & 0.00 & 214.88 & 68.93 & 12704 & 28280 & 497927 \\
\hline $\mathrm{c} 25202$ & 213.19 & 0.39 & 129.04 & 3851.21 & 30295 & 32280 & 505552 \\
\hline $\mathrm{c} 25204$ & 213.19 & 0.46 & 114.93 & 3601.28 & 691 & 35380 & 510827 \\
\hline $\mathrm{c} 25205$ & 214.67 & 0.50 & 106.48 & 3601.35 & 629 & 36480 & 512877 \\
\hline $\mathrm{c} 25206$ & 232.58 & 0.36 & 138.11 & 3600.68 & 16042 & 29705 & 500327 \\
\hline $\mathrm{c} 25207$ & 214.67 & 0.48 & 121.74 & 3600.69 & 6129 & 30480 & 501702 \\
\hline $\mathrm{c} 25208$ & 214.88 & 0.48 & 135.96 & 3602.05 & 1734 & 32155 & 504577 \\
\hline
\end{tabular}

Table 29 - Results for the class of instances C, with 50\% drone speed and 75\% drone eligible weight, using the HGA as a starting solution.

\begin{tabular}{l|c|c|c|c|c|c|c}
\hline Instance & $\begin{array}{c}\text { Objective } \\
\text { Function }\end{array}$ & GAP & Dual & Exec. Time & Nodes & Variables & Constraints \\
\hline $\mathrm{c} 25101$ & 191.81 & 0.00 & 191.81 & 6.98 & 0 & 35180 & 506702 \\
\hline $\mathrm{c} 25102$ & 190.74 & 0.28 & 137.87 & 3600.43 & 5 & 45680 & 520827 \\
\hline $\mathrm{c} 25103$ & 192.54 & 0.82 & 35.42 & 3601.40 & 43 & 49030 & 526552 \\
\hline $\mathrm{c} 25104$ & 187.45 & 0.90 & 18.33 & 3601.51 & 1 & 51555 & 529952 \\
\hline $\mathrm{c} 25105$ & 191.81 & 0.00 & 191.81 & 29.96 & 0 & 38330 & 510552 \\
\hline $\mathrm{c} 25106$ & 191.81 & 0.00 & 191.81 & 7.44 & 0 & 36005 & 507627 \\
\hline $\mathrm{c} 25107$ & 191.81 & 0.33 & 128.69 & 3600.88 & 812 & 39180 & 511902 \\
\hline $\mathrm{c} 25109$ & 191.81 & 0.86 & 26.99 & 3600.99 & 3168 & 42555 & 516627 \\
\hline $\mathrm{c} 25201$ & 2141.81 & 0.89 & 20.20 & 3600.89 & 4 & 45180 & 520527 \\
\hline $\mathrm{c} 25202$ & 214.88 & 0.03 & 215.54 & 19.32 & 0 & 28880 & 501002 \\
\hline $\mathrm{c} 25203$ & 214.88 & 0.53 & 126.46 & 3600.65 & 43520 & 32880 & 508627 \\
\hline $\mathrm{c} 25205$ & 218.88 & 0.58 & 91.88 & 3605.83 & 284 & 35980 & 513877 \\
\hline $\mathrm{c} 25206$ & 232.55 & 0.43 & 133.40 & 3600.57 & 436 & 37080 & 515927 \\
\hline $\mathrm{c} 25207$ & 214.88 & 0.49 & 119.47 & 3614.68 & 9854 & 30355 & 503427 \\
\hline $\mathrm{c} 25208$ & 215.54 & 0.49 & 116.66 & 3601.25 & 2431 & 32805 & 504802 \\
\hline
\end{tabular}


Table 30 - Results for the class of instances C, with 100\% drone speed and 25\% drone eligible weight, using the HGA as a starting solution.

\begin{tabular}{l|c|c|c|c|c|c|c}
\hline Instance & $\begin{array}{c}\text { Objective } \\
\text { Function }\end{array}$ & GAP & Dual & Exec. Time & Nodes & Variables & Constraints \\
\hline $\mathrm{c} 25101$ & 181.49 & $<0.01$ & 181.49 & 75.21 & 15839 & 41405 & 500677 \\
\hline $\mathrm{c} 25102$ & 190.72 & 0.40 & 114.88 & 3601.02 & 3 & 54705 & 517627 \\
\hline $\mathrm{c} 25103$ & 188.26 & 0.45 & 103.26 & 3601.70 & 2 & 58805 & 524127 \\
\hline $\mathrm{c} 25104$ & 184.06 & 0.43 & 104.33 & 3601.49 & 5 & 62855 & 529052 \\
\hline $\mathrm{c} 25105$ & 177.93 & $<0.01$ & 177.92 & 96.36 & 12460 & 44955 & 504902 \\
\hline $\mathrm{c} 25106$ & 177.93 & 0.00 & 177.93 & 21.78 & 5 & 42455 & 501827 \\
\hline $\mathrm{c} 25107$ & 177.93 & 0.06 & 166.77 & 3600.59 & 90196 & 46030 & 506477 \\
\hline $\mathrm{c} 25108$ & 186.82 & 0.72 & 52.13 & 3601.47 & 1394 & 50455 & 512302 \\
\hline $\mathrm{c} 25209$ & 184.13 & 0.73 & 48.90 & 3601.24 & 6 & 53855 & 517002 \\
\hline $\mathrm{c} 25202$ & 209.89 & 0.00 & 209.89 & 24.58 & 1223 & 41455 & 501327 \\
\hline $\mathrm{c} 25203$ & 210.83 & 0.39 & 128.49 & 3601.27 & 6031 & 49155 & 512677 \\
\hline $\mathrm{c} 25204$ & 212.81 & 0.50 & 106.30 & 3601.55 & 8 & 55530 & 521227 \\
\hline $\mathrm{c} 25205$ & 212.56 & 0.51 & 104.36 & 3602.30 & 2 & 57180 & 523827 \\
\hline $\mathrm{c} 25206$ & 212.52 & 0.37 & 133.43 & 3600.70 & 9918 & 45680 & 506527 \\
\hline $\mathrm{c} 25207$ & 212.32 & 0.50 & 111.80 & 3601.18 & 4508 & 47280 & 508752 \\
\hline $\mathrm{c} 25208$ & 212.52 & 0.50 & 104.73 & 3602.58 & 2572 & 50155 & 512827 \\
\hline & & & & & & & 510802 \\
\hline
\end{tabular}

Table 31 - Results for the class of instances C, with 100\% drone speed and 75\% drone eligible weight, using the HGA as a starting solution.

\begin{tabular}{l|c|c|c|c|c|c|c}
\hline Instance & $\begin{array}{c}\text { Objective } \\
\text { Function }\end{array}$ & GAP & Dual & Exec. Time & Nodes & Variables & Constraints \\
\hline $\mathrm{c} 25101$ & 181.46 & 0.00 & 181.45 & 334.54 & 27555 & 54305 & 525877 \\
\hline $\mathrm{c} 25102$ & 188.98 & 0.76 & 46.07 & 3601.93 & 12 & 73005 & 548202 \\
\hline $\mathrm{c} 25103$ & 188.12 & 0.92 & 15.15 & 3602.25 & 1 & 78930 & 556502 \\
\hline $\mathrm{c} 25104$ & 182.73 & 0.92 & 14.51 & 3603.55 & 0 & 85055 & 563502 \\
\hline $\mathrm{c} 25105$ & 184.10 & 0.34 & 122.04 & 3600.65 & 1365 & 60480 & 532752 \\
\hline $\mathrm{c} 25106$ & 177.90 & 0.00 & 177.90 & 1069.93 & 29012 & 55855 & 527527 \\
\hline $\mathrm{c} 25107$ & 186.47 & 0.55 & 83.42 & 3602.92 & 18 & 61555 & 534327 \\
\hline $\mathrm{c} 25109$ & 184.10 & 0.91 & 17.27 & 3601.42 & 4 & 67480 & 541602 \\
\hline $\mathrm{c} 25201$ & 184.10 & 0.92 & 15.32 & 3602.07 & 1 & 72955 & 548352 \\
\hline $\mathrm{c} 25202$ & 201.88 & 0.00 & 201.87 & 44.31 & 222 & 57630 & 529802 \\
\hline $\mathrm{c} 25203$ & 205.73 & 0.61 & 80.26 & 3601.03 & 15 & 68355 & 544152 \\
\hline $\mathrm{c} 25204$ & 205.38 & 0.81 & 39.09 & 3601.97 & 0 & 76780 & 554727 \\
\hline $\mathrm{c} 25206$ & 205.11 & 0.57 & 83.36 & 3601.44 & 0 & 80705 & 559602 \\
\hline $\mathrm{c} 25207$ & 205.11 & 0.81 & 39.87 & 3600.64 & 4 & 64005 & 537127 \\
\hline $\mathrm{c} 25208$ & 205.25 & 0.76 & 49.56 & 3602.48 & 10 & 66655 & 540377 \\
\hline & & 0.81 & 38.45 & 3602.60 & 0 & 69480 & 546652 \\
\hline
\end{tabular}


Table 32 - Results for the class of instances C, with 150\% drone speed and 25\% drone eligible weight, using the HGA as a starting solution.

\begin{tabular}{l|c|c|c|c|c|c|c}
\hline Instance & $\begin{array}{c}\text { Objective } \\
\text { Function }\end{array}$ & GAP & Dual & Exec. Time & Nodes & Variables & Constraints \\
\hline $\mathrm{c} 25101$ & 173.06 & 0.00 & 173.06 & 27.06 & 0 & 72705 & 532077 \\
\hline $\mathrm{c} 25102$ & 189.39 & 0.39 & 114.89 & 3601.48 & 4 & 101505 & 564452 \\
\hline $\mathrm{c} 25103$ & 187.52 & 0.45 & 103.60 & 3605.90 & 127 & 111555 & 576877 \\
\hline $\mathrm{c} 25104$ & 183.29 & 0.44 & 103.26 & 3601.39 & 0 & 118355 & 584552 \\
\hline $\mathrm{c} 25105$ & 179.14 & 0.07 & 166.64 & 3600.98 & 27752 & 80505 & 540502 \\
\hline $\mathrm{c} 25106$ & 173.06 & 0.00 & 173.06 & 36.51 & 1 & 74280 & 533752 \\
\hline $\mathrm{c} 25107$ & 184.13 & 0.10 & 165.59 & 3601.11 & 3 & 83430 & 543927 \\
\hline $\mathrm{c} 25108$ & 186.81 & 0.70 & 56.13 & 3602.92 & 355 & 91930 & 553802 \\
\hline $\mathrm{c} 25109$ & 182.26 & 0.73 & 48.90 & 3603.31 & 2 & 99030 & 562177 \\
\hline $\mathrm{c} 25201$ & 197.00 & 0.00 & 197.00 & 502.34 & 19769 & 75355 & 535252 \\
\hline $\mathrm{c} 25202$ & 220.44 & 0.43 & 125.16 & 3601.87 & 10 & 94830 & 558377 \\
\hline $\mathrm{c} 25203$ & 220.73 & 0.52 & 105.89 & 3604.15 & 7 & 110605 & 576302 \\
\hline $\mathrm{c} 25204$ & 217.11 & 0.52 & 104.36 & 3601.64 & 0 & 113180 & 579827 \\
\hline $\mathrm{c} 25205$ & 210.37 & 0.35 & 136.86 & 3602.11 & 29728 & 83405 & 544277 \\
\hline $\mathrm{c} 25206$ & 212.52 & 0.47 & 112.35 & 3601.75 & 12434 & 86705 & 548177 \\
\hline $\mathrm{c} 25207$ & 212.52 & 0.38 & 130.88 & 3604.31 & 299 & 93705 & 556377 \\
\hline $\mathrm{c} 25208$ & 212.52 & 0.51 & 104.42 & 3602.44 & 1722 & 89655 & 551702 \\
\hline
\end{tabular}

Table 33 - Results for the class of instances C, with $150 \%$ drone speed and 50\% drone eligible weight, using the HGA as a starting solution.

\begin{tabular}{l|c|c|c|c|c|c|c}
\hline Instance & $\begin{array}{c}\text { Objective } \\
\text { Function }\end{array}$ & GAP & Dual & Exec. Time & Nodes & Variables & Constraints \\
\hline $\mathrm{c} 25101$ & 159.65 & 0.00 & 159.65 & 69.83 & 8281 & 102805 & 572002 \\
\hline $\mathrm{c} 25102$ & 189.24 & 0.43 & 107.12 & 3602.81 & 1 & 143605 & 616352 \\
\hline $\mathrm{c} 25103$ & 187.38 & 0.58 & 79.61 & 3600.82 & 0 & 158180 & 633302 \\
\hline $\mathrm{c} 25104$ & 183.05 & 0.57 & 79.61 & 3602.21 & 0 & 169455 & 645452 \\
\hline $\mathrm{c} 25105$ & 186.80 & 0.34 & 122.78 & 3810.11 & 13779 & 114905 & 584727 \\
\hline $\mathrm{c} 25106$ & 159.65 & 0.00 & 159.65 & 498.48 & 24984 & 105330 & 574627 \\
\hline $\mathrm{c} 25107$ & 186.47 & 0.43 & 106.35 & 3601.56 & 1 & 118380 & 588702 \\
\hline $\mathrm{c} 25109$ & 184.10 & 0.86 & 26.00 & 3602.67 & 1 & 130505 & 602177 \\
\hline $\mathrm{c} 25201$ & 182.25 & 0.88 & 22.77 & 3601.35 & 0 & 142105 & 615052 \\
\hline $\mathrm{c} 25202$ & 205.15 & 0.41 & 110.60 & 3600.63 & 11276 & 111855 & 581577 \\
\hline $\mathrm{c} 25203$ & 205.38 & 0.70 & 60.94 & 3602.69 & 3 & 137330 & 610677 \\
\hline $\mathrm{c} 25205$ & 206.36 & 0.78 & 49.93 & 3603.42 & 0 & 157780 & 633277 \\
\hline $\mathrm{c} 25206$ & 205.11 & 0.75 & 51.31 & 3601.45 & 0 & 166055 & 642502 \\
\hline $\mathrm{c} 25207$ & 205.11 & 0.79 & 43.22 & 3601.13 & 3 & 123430 & 594102 \\
\hline $\mathrm{c} 25208$ & 205.11 & 0.76 & 48.81 & 36.73 & 2 & 129080 & 600352 \\
\hline
\end{tabular}


Table 34 - Results for the class of instances C, with 150\% drone speed and 75\% drone eligible weight, using the HGA as a starting solution.

\begin{tabular}{l|c|c|c|c|c|c|c}
\hline Instance & $\begin{array}{c}\text { Objective } \\
\text { Function }\end{array}$ & GAP & Dual & Exec. Time & Nodes & Variables & Constraints \\
\hline $\mathrm{c} 25101$ & 157.79 & 0.00 & 157.79 & 85.67 & 5525 & 110030 & 581677 \\
\hline $\mathrm{c} 25102$ & 188.00 & 0.76 & 44.67 & 3603.22 & 1 & 152880 & 628077 \\
\hline $\mathrm{c} 25103$ & 188.54 & 0.93 & 14.07 & 3602.32 & 0 & 169630 & 647202 \\
\hline $\mathrm{c} 25104$ & 183.64 & 1.00 & 0.00 & 4000.09 & 0 & 182105 & 660552 \\
\hline $\mathrm{c} 25105$ & 186.77 & 0.51 & 91.48 & 3601.19 & 6 & 123880 & 596152 \\
\hline $\mathrm{c} 25106$ & 157.79 & 0.00 & 157.78 & 899.62 & 21373 & 112555 & 584302 \\
\hline $\mathrm{c} 25107$ & 184.10 & 0.59 & 75.86 & 3601.88 & 2 & 127980 & 600752 \\
\hline $\mathrm{c} 25108$ & 184.09 & 0.92 & 14.48 & 3602.65 & 1 & 140905 & 615027 \\
\hline $\mathrm{c} 25109$ & 182.25 & 0.92 & 14.29 & 3600.16 & 0 & 154155 & 629552 \\
\hline $\mathrm{c} 25201$ & 201.36 & 0.50 & 100.26 & 3602.99 & 9530 & 121155 & 593327 \\
\hline $\mathrm{c} 25202$ & 207.12 & 0.77 & 48.34 & 3603.21 & 1 & 152530 & 628327 \\
\hline $\mathrm{c} 25203$ & 205.38 & 0.83 & 34.26 & 3606.14 & 0 & 172980 & 650927 \\
\hline $\mathrm{c} 25204$ & 206.68 & 0.85 & 30.18 & 3601.47 & 0 & 181705 & 660602 \\
\hline $\mathrm{c} 25205$ & 205.11 & 0.81 & 38.08 & 3601.20 & 4 & 133705 & 606827 \\
\hline $\mathrm{c} 25206$ & 205.11 & 0.87 & 27.61 & 3601.87 & 3 & 139805 & 613527 \\
\hline $\mathrm{c} 25207$ & 205.46 & 0.85 & 29.86 & 3601.03 & 0 & 151680 & 626602 \\
\hline $\mathrm{c} 25208$ & 206.88 & 0.86 & 29.07 & 3602.34 & 1 & 145255 & 619552 \\
\hline
\end{tabular}

Table 35 - Results for the class of instances $\mathrm{R}$, with $50 \%$ drone speed and $25 \%$ drone eligible weight, using the HGA as a starting solution.

\begin{tabular}{l|c|c|c|c|c|c|c}
\hline Instance & $\begin{array}{c}\text { Objective } \\
\text { Function }\end{array}$ & GAP & Dual & Exec. Time & Nodes & Variables & Constraints \\
\hline $\mathrm{r} 25101$ & 612.62 & 0.00 & 612.62 & 24.35 & 2314 & 22005 & 470127 \\
\hline $\mathrm{r} 25102$ & 548.11 & 0.32 & 375.16 & 3605.75 & 481825 & 26755 & 479652 \\
\hline $\mathrm{r} 25103$ & 464.83 & 0.36 & 296.89 & 3611.98 & 459436 & 29555 & 485252 \\
\hline $\mathrm{r} 25104$ & 439.76 & 0.34 & 292.27 & 3611.86 & 551877 & 31005 & 488152 \\
\hline $\mathrm{r} 25105$ & 525.83 & 0.00 & 525.79 & 87.27 & 29803 & 23905 & 473927 \\
\hline $\mathrm{r} 25106$ & 470.33 & 0.33 & 315.61 & 3611.22 & 462802 & 28005 & 482152 \\
\hline $\mathrm{r} 25107$ & 431.81 & 0.30 & 300.20 & 3609.04 & 484477 & 30205 & 486552 \\
\hline $\mathrm{r} 25108$ & 398.94 & 0.29 & 282.65 & 3612.51 & 513980 & 31480 & 489102 \\
\hline $\mathrm{r} 25109$ & 433.66 & 0.00 & 433.62 & 680.22 & 200485 & 26680 & 479477 \\
\hline $\mathrm{r} 25110$ & 441.17 & 0.35 & 284.89 & 3609.95 & 484022 & 29705 & 485552 \\
\hline $\mathrm{r} 25111$ & 408.76 & 0.25 & 305.74 & 3602.84 & 637250 & 29605 & 485352 \\
\hline $\mathrm{r} 25112$ & 401.86 & 0.30 & 280.75 & 3633.41 & 408871 & 32705 & 491552 \\
\hline $\mathrm{r} 25201$ & 454.61 & 0.00 & 454.61 & 111.24 & 29739 & 26380 & 478877 \\
\hline $\mathrm{r} 25202$ & 441.83 & 0.32 & 298.88 & 3611.07 & 645111 & 29430 & 485002 \\
\hline $\mathrm{r} 25203$ & 392.33 & 0.26 & 289.06 & 3614.02 & 470152 & 31380 & 488902 \\
\hline $\mathrm{r} 25204$ & 356.57 & 0.21 & 280.93 & 3608.47 & 503661 & 32230 & 490602 \\
\hline $\mathrm{r} 25206$ & 377.51 & 0.00 & 377.49 & 228.49 & 50212 & 28655 & 483427 \\
\hline $\mathrm{r} 25207$ & 375.48 & 0.24 & 285.00 & 3607.37 & 479607 & 30605 & 487352 \\
\hline $\mathrm{r} 25208$ & 340.53 & 0.22 & 282.37 & 3623.68 & 473070 & 31705 & 489552 \\
\hline $\mathrm{r} 25209$ & 375.97 & 0.18 & 279.83 & 3609.01 & 479394 & 32330 & 490802 \\
\hline $\mathrm{r} 25210$ & 405.48 & 0.27 & 297.10 & 3606.36 & 619063 & 30155 & 486452 \\
\hline $\mathrm{r} 25211$ & 352.50 & 0.21 & 279.31 & 367.79 & 486405 & 30480 & 487102 \\
\hline
\end{tabular}


Table 36 - Results for the class of instances R, with 50\% drone speed and 50\% drone eligible weight, using the HGA as a starting solution.

\begin{tabular}{|c|c|c|c|c|c|c|c|}
\hline Instance & $\begin{array}{l}\text { Objective } \\
\text { Function }\end{array}$ & GAP & Dual & Exec. Time & Nodes & Variables & Constraints \\
\hline r25101 & 612.62 & 0.00 & 612.62 & 16.57 & 1 & 22005 & 478702 \\
\hline r25102 & 548.11 & 0.32 & 374.25 & 3605.24 & 202328 & 26805 & 488377 \\
\hline r25103 & 462.93 & 0.33 & 308.23 & 3616.03 & 464687 & 29630 & 494052 \\
\hline r25104 & 436.52 & 0.36 & 278.63 & 3606.30 & 405455 & 31105 & 497027 \\
\hline $\mathrm{r} 25105$ & 525.79 & 0.00 & 525.74 & 38.99 & 13086 & 23930 & 482577 \\
\hline r25106 & 468.68 & 0.33 & 312.66 & 3606.86 & 430404 & 28080 & 490952 \\
\hline r25107 & 431.81 & 0.32 & 293.48 & 3606.72 & 454842 & 30280 & 495352 \\
\hline r25108 & 451.10 & 0.39 & 274.13 & 3606.42 & 364196 & 31580 & 497977 \\
\hline r25109 & 430.40 & 0.00 & 430.36 & 530.91 & 152242 & 26730 & 488202 \\
\hline r25110 & 448.25 & 0.36 & 287.52 & 3699.84 & 255028 & 29805 & 494427 \\
\hline r25111 & 440.05 & 0.36 & 281.57 & 3619.82 & 275579 & 29680 & 494152 \\
\hline r25112 & 404.60 & 0.34 & 265.53 & 3612.38 & 383572 & 32805 & 500427 \\
\hline r25201 & 454.61 & 0.00 & 454.60 & 104.57 & 30115 & 26405 & 487527 \\
\hline r25202 & 411.49 & 0.27 & 300.86 & 3609.53 & 512960 & 29505 & 493802 \\
\hline r25203 & 394.70 & 0.27 & 289.56 & 3613.52 & 475923 & 31455 & 497702 \\
\hline r25204 & 366.95 & 0.28 & 265.20 & 3607.71 & 425110 & 32330 & 499477 \\
\hline r25205 & 377.51 & 0.13 & 328.06 & 3614.46 & 584204 & 28705 & 492152 \\
\hline r25206 & 375.48 & 0.24 & 284.09 & 3608.76 & 535266 & 30680 & 496152 \\
\hline r25207 & 362.63 & 0.23 & 279.96 & 3612.92 & 618728 & 31780 & 498352 \\
\hline r25208 & 347.80 & 0.24 & 265.53 & 3614.34 & 452830 & 32430 & 499677 \\
\hline $\mathrm{r} 25209$ & 373.06 & 0.17 & 309.55 & 3600.73 & 851888 & 30205 & 495177 \\
\hline r25210 & 412.80 & 0.24 & 315.46 & 3609.01 & 353067 & 30555 & 495902 \\
\hline r25211 & 350.44 & 0.25 & 263.53 & 3606.94 & 389855 & 32830 & 500477 \\
\hline
\end{tabular}

Table 37 - Results for the class of instances R, with 50\% drone speed and 75\% drone eligible weight, using the HGA as a starting solution.

\begin{tabular}{l|c|c|c|c|c|c|c}
\hline Instance & $\begin{array}{c}\text { Objective } \\
\text { Function }\end{array}$ & GAP & Dual & Exec. Time & Nodes & Variables & Constraints \\
\hline $\mathrm{r} 25101$ & 612.62 & 0.00 & 612.62 & 21.25 & 3 & 22030 & 484902 \\
\hline $\mathrm{r} 25102$ & 548.11 & 0.32 & 370.58 & 3606.38 & 477836 & 26855 & 494652 \\
\hline $\mathrm{r} 25103$ & 455.70 & 0.34 & 302.01 & 3608.15 & 554220 & 29680 & 500327 \\
\hline $\mathrm{r} 25104$ & 422.34 & 0.37 & 266.77 & 3620.17 & 441764 & 31155 & 503302 \\
\hline $\mathrm{r} 25105$ & 522.54 & 0.00 & 522.54 & 46.83 & 27723 & 23980 & 488852 \\
\hline $\mathrm{r} 25106$ & 494.41 & 0.37 & 313.31 & 3612.52 & 447825 & 28130 & 497227 \\
\hline $\mathrm{r} 25107$ & 431.81 & 0.32 & 292.26 & 3608.98 & 529671 & 30330 & 501627 \\
\hline $\mathrm{r} 25108$ & 398.94 & 0.32 & 270.18 & 3618.35 & 383311 & 31630 & 504252 \\
\hline $\mathrm{r} 25109$ & 430.40 & 0.00 & 430.36 & 975.83 & 280650 & 26780 & 494477 \\
\hline $\mathrm{r} 25110$ & 447.47 & 0.38 & 278.36 & 3668.23 & 193969 & 29855 & 500702 \\
\hline $\mathrm{r} 25111$ & 440.05 & 0.36 & 281.57 & 3645.98 & 330001 & 29730 & 500427 \\
\hline $\mathrm{r} 25112$ & 395.41 & 0.36 & 254.94 & 3600.01 & 289541 & 32855 & 506702 \\
\hline $\mathrm{r} 25201$ & 452.90 & 0.00 & 452.90 & 46.57 & 14777 & 26455 & 493802 \\
\hline $\mathrm{r} 25202$ & 411.49 & 0.27 & 299.54 & 3611.50 & 550056 & 29555 & 500077 \\
\hline $\mathrm{r} 25203$ & 392.33 & 0.26 & 289.66 & 3614.91 & 504403 & 31505 & 503977 \\
\hline $\mathrm{r} 25204$ & 355.14 & 0.27 & 258.40 & 3613.44 & 425830 & 32380 & 505752 \\
\hline $\mathrm{r} 25205$ & 374.26 & 0.00 & 374.23 & 355.36 & 105927 & 28755 & 498427 \\
\hline $\mathrm{r} 25206$ & 375.48 & 0.25 & 282.64 & 3611.47 & 362634 & 30730 & 502427 \\
\hline $\mathrm{r} 25207$ & 361.61 & 0.23 & 278.86 & 3618.00 & 536119 & 31830 & 504627 \\
\hline $\mathrm{r} 25208$ & 352.79 & 0.29 & 251.92 & 3616.95 & 471969 & 32480 & 505952 \\
\hline $\mathrm{r} 25209$ & 370.48 & 0.17 & 309.32 & 3602.20 & 864048 & 30255 & 501452 \\
\hline $\mathrm{r} 25210$ & 405.48 & 0.25 & 305.97 & 3609.28 & 468854 & 30605 & 502177 \\
\hline $\mathrm{r} 25211$ & 352.45 & 0.27 & 256.44 & 3621.34 & 294696 & 32880 & 506752 \\
\hline
\end{tabular}


Table 38 - Results for the class of instances R, with 100\% drone speed and 25\% drone eligible weight, using the HGA as a starting solution.

\begin{tabular}{l|c|c|c|c|c|c|c}
\hline Instance & $\begin{array}{c}\text { Objective } \\
\text { Function }\end{array}$ & GAP & Dual & Exec. Time & Nodes & Variables & Constraints \\
\hline $\mathrm{r} 25101$ & 595.04 & 0.00 & 595.04 & 5.22 & 0 & 27030 & 476152 \\
\hline $\mathrm{r} 25102$ & 540.37 & 0.40 & 326.86 & 3619.86 & 10656 & 35055 & 489027 \\
\hline $\mathrm{r} 25103$ & 434.57 & 0.43 & 247.82 & 3601.62 & 4995 & 38005 & 494777 \\
\hline $\mathrm{r} 25104$ & 411.65 & 0.46 & 221.34 & 3601.11 & 2 & 40380 & 498527 \\
\hline $\mathrm{r} 25105$ & 485.48 & 0.23 & 373.01 & 3607.85 & 41506 & 30530 & 481602 \\
\hline $\mathrm{r} 25106$ & 461.03 & 0.43 & 262.86 & 3601.20 & 1358 & 36830 & 492052 \\
\hline $\mathrm{r} 25107$ & 445.93 & 0.47 & 237.06 & 3601.46 & 5239 & 39030 & 496452 \\
\hline $\mathrm{r} 25108$ & 380.40 & 0.42 & 221.34 & 3601.86 & 276 & 40880 & 499502 \\
\hline $\mathrm{r} 25109$ & 428.14 & 0.35 & 277.49 & 3615.30 & 3658 & 33755 & 487577 \\
\hline $\mathrm{r} 25110$ & 413.54 & 0.40 & 248.17 & 3601.49 & 1412 & 38405 & 495202 \\
\hline $\mathrm{r} 25111$ & 395.03 & 0.38 & 243.84 & 3602.77 & 2671 & 38355 & 495177 \\
\hline $\mathrm{r} 25112$ & 372.74 & 0.39 & 228.56 & 3601.42 & 1794 & 42105 & 501952 \\
\hline $\mathrm{r} 25201$ & 435.36 & 0.15 & 370.24 & 3613.19 & 311358 & 32980 & 486502 \\
\hline $\mathrm{r} 25202$ & 390.08 & 0.34 & 255.80 & 3601.04 & 3576 & 38255 & 494902 \\
\hline $\mathrm{r} 25203$ & 372.23 & 0.34 & 243.94 & 3601.45 & 211 & 40205 & 498802 \\
\hline $\mathrm{r} 25204$ & 355.62 & 0.35 & 230.10 & 3601.66 & 911 & 41630 & 501002 \\
\hline $\mathrm{r} 25205$ & 371.26 & 0.22 & 290.27 & 3609.76 & 17913 & 35830 & 491652 \\
\hline $\mathrm{r} 25206$ & 340.01 & 0.32 & 230.67 & 3601.05 & 4069 & 39455 & 497277 \\
\hline $\mathrm{r} 25207$ & 341.97 & 0.34 & 226.82 & 3601.27 & 2914 & 40555 & 499477 \\
\hline $\mathrm{r} 25208$ & 323.94 & 0.31 & 222.13 & 3602.28 & 351 & 41730 & 501202 \\
\hline $\mathrm{r} 25209$ & 362.09 & 0.33 & 243.06 & 3601.42 & 709 & 38555 & 495852 \\
\hline $\mathrm{r} 25210$ & 382.81 & 0.30 & 267.53 & 3601.36 & 2088 & 39105 & 496777 \\
\hline $\mathrm{r} 25211$ & 335.97 & 0.32 & 229.17 & 3601.94 & 1678 & 42130 & 502002 \\
\hline
\end{tabular}

Table 39 - Results for the class of instances R, with 100\% drone speed and 75\% drone eligible weight, using the HGA as a starting solution.

\begin{tabular}{l|c|c|c|c|c|c|c}
\hline Instance & $\begin{array}{c}\text { Objective } \\
\text { Function }\end{array}$ & GAP & Dual & Exec. Time & Nodes & Variables & Constraints \\
\hline $\mathrm{r} 25101$ & 478.69 & 0.00 & 478.69 & 123.89 & 16636 & 34305 & 498252 \\
\hline $\mathrm{r} 25102$ & 487.52 & 0.70 & 141.43 & 3600.84 & 1 & 45730 & 514477 \\
\hline $\mathrm{r} 25103$ & 393.07 & 0.70 & 119.12 & 3601.92 & 1 & 50905 & 522427 \\
\hline $\mathrm{r} 25104$ & 370.39 & 0.70 & 112.65 & 3602.38 & 1 & 54330 & 527227 \\
\hline $\mathrm{r} 25105$ & 446.31 & 0.47 & 236.24 & 3600.42 & 8 & 40005 & 505877 \\
\hline $\mathrm{r} 25106$ & 425.68 & 0.70 & 127.74 & 3601.69 & 1 & 48730 & 518702 \\
\hline $\mathrm{r} 25107$ & 378.70 & 0.69 & 119.00 & 3602.28 & 1 & 52105 & 524277 \\
\hline $\mathrm{r} 25108$ & 340.30 & 0.67 & 112.49 & 3601.09 & 0 & 54980 & 528352 \\
\hline $\mathrm{r} 25109$ & 349.11 & 0.56 & 154.55 & 3601.50 & 1 & 44755 & 513402 \\
\hline $\mathrm{r} 25110$ & 368.16 & 0.68 & 117.06 & 3602.05 & 1 & 51880 & 523477 \\
\hline $\mathrm{r} 25111$ & 371.74 & 0.68 & 119.04 & 3602.17 & 1 & 51030 & 522602 \\
\hline $\mathrm{r} 25112$ & 303.47 & 0.64 & 107.97 & 3601.05 & 0 & 56430 & 531027 \\
\hline $\mathrm{r} 25201$ & 411.25 & 0.32 & 279.05 & 3633.29 & 36287 & 42430 & 510752 \\
\hline $\mathrm{r} 25202$ & 365.85 & 0.63 & 133.61 & 3601.31 & 2 & 50255 & 521652 \\
\hline $\mathrm{r} 25203$ & 348.47 & 0.66 & 119.50 & 3602.07 & 2 & 53355 & 526702 \\
\hline $\mathrm{r} 25204$ & 327.51 & 0.66 & 112.49 & 3602.69 & 1 & 55755 & 529877 \\
\hline $\mathrm{r} 25205$ & 354.47 & 0.49 & 178.70 & 3601.05 & 3 & 47430 & 518052 \\
\hline $\mathrm{r} 25206$ & 324.13 & 0.65 & 114.95 & 3601.98 & 1 & 52280 & 524852 \\
\hline $\mathrm{r} 25207$ & 326.87 & 0.65 & 113.33 & 3602.41 & 1 & 53980 & 527652 \\
\hline $\mathrm{r} 25208$ & 290.39 & 0.61 & 112.40 & 3602.22 & 1 & 55930 & 530152 \\
\hline $\mathrm{r} 25209$ & 306.62 & 0.57 & 131.34 & 3601.83 & 4 & 51005 & 523077 \\
\hline $\mathrm{r} 25210$ & 363.76 & 0.58 & 152.12 & 3601.83 & 2 & 51605 & 524027 \\
\hline $\mathrm{r} 25211$ & 315.78 & 0.66 & 107.97 & 3601.11 & 0 & 56455 & 531077 \\
\hline
\end{tabular}


Table 40 - Results for the class of instances R, with 150\% drone speed and 25\% drone eligible weight, using the HGA as a starting solution.

\begin{tabular}{l|c|c|c|c|c|c|c}
\hline Instance & $\begin{array}{c}\text { Objective } \\
\text { Function }\end{array}$ & GAP & Dual & Exec. Time & Nodes & Variables & Constraints \\
\hline $\mathrm{r} 25101$ & 542.28 & 0.00 & 542.28 & 35.66 & 829 & 41755 & 491002 \\
\hline $\mathrm{r} 25102$ & 507.20 & 0.38 & 312.91 & 3602.78 & 4539 & 59505 & 513552 \\
\hline $\mathrm{r} 25103$ & 423.62 & 0.43 & 239.47 & 3602.07 & 15 & 63080 & 519902 \\
\hline $\mathrm{r} 25104$ & 374.25 & 0.39 & 229.63 & 3605.17 & 267 & 67255 & 525452 \\
\hline $\mathrm{r} 25105$ & 454.12 & 0.21 & 359.35 & 3600.77 & 19269 & 48305 & 499502 \\
\hline $\mathrm{r} 25106$ & 428.56 & 0.39 & 260.50 & 3601.71 & 18 & 62030 & 517302 \\
\hline $\mathrm{r} 25107$ & 400.46 & 0.39 & 243.76 & 3602.62 & 16 & 64680 & 522152 \\
\hline $\mathrm{r} 25108$ & 363.98 & 0.37 & 229.63 & 3604.70 & 357 & 68880 & 526552 \\
\hline $\mathrm{r} 25109$ & 427.65 & 0.35 & 276.53 & 3603.06 & 3359 & 55030 & 508977 \\
\hline $\mathrm{r} 25110$ & 399.80 & 0.38 & 247.44 & 3604.12 & 793 & 62905 & 519802 \\
\hline $\mathrm{r} 25111$ & 394.71 & 0.40 & 237.06 & 3604.10 & 3 & 64455 & 521327 \\
\hline $\mathrm{r} 25112$ & 351.62 & 0.33 & 234.63 & 3606.03 & 595 & 69105 & 529002 \\
\hline $\mathrm{r} 25201$ & 394.50 & 0.08 & 362.00 & 3600.45 & 81693 & 50905 & 504552 \\
\hline $\mathrm{r} 25202$ & 390.08 & 0.36 & 248.76 & 3602.95 & 245 & 63555 & 520252 \\
\hline $\mathrm{r} 25203$ & 372.01 & 0.36 & 237.06 & 3603.08 & 1 & 65855 & 524502 \\
\hline $\mathrm{r} 25204$ & 395.06 & 0.44 & 222.37 & 3605.81 & 947 & 68630 & 528052 \\
\hline $\mathrm{r} 25205$ & 334.25 & 0.20 & 266.72 & 3601.26 & 16772 & 56730 & 512652 \\
\hline $\mathrm{r} 25206$ & 337.63 & 0.31 & 233.29 & 3604.17 & 495 & 65280 & 523152 \\
\hline $\mathrm{r} 25207$ & 337.49 & 0.33 & 226.55 & 3604.60 & 1021 & 66555 & 525527 \\
\hline $\mathrm{r} 25208$ & 323.86 & 0.30 & 226.74 & 3607.10 & 1116 & 68730 & 528252 \\
\hline $\mathrm{r} 25209$ & 368.33 & 0.31 & 252.38 & 3604.03 & 160 & 61855 & 519227 \\
\hline $\mathrm{r} 25210$ & 382.81 & 0.38 & 237.06 & 3601.97 & 7 & 64305 & 522052 \\
\hline $\mathrm{r} 25211$ & 308.63 & 0.27 & 224.01 & 3607.58 & 543 & 69130 & 529052 \\
\hline
\end{tabular}

Table 41 - Results for the class of instances R, with 150\% drone speed and 50\% drone eligible weight, using the HGA as a starting solution.

\begin{tabular}{l|c|c|c|c|c|c|c}
\hline Instance & $\begin{array}{c}\text { Objective } \\
\text { Function }\end{array}$ & GAP & Dual & Exec. Time & Nodes & Variables & Constraints \\
\hline $\mathrm{r} 25101$ & 330.34 & 0.00 & 330.34 & 193.37 & 9926 & 62355 & 520177 \\
\hline $\mathrm{r} 25102$ & 426.92 & 0.53 & 202.38 & 3602.37 & 2 & 90505 & 553127 \\
\hline $\mathrm{r} 25103$ & 388.43 & 0.53 & 182.06 & 3601.52 & 0 & 99255 & 564652 \\
\hline $\mathrm{r} 25104$ & 342.58 & 0.51 & 167.97 & 3601.77 & 0 & 105830 & 572602 \\
\hline $\mathrm{r} 25105$ & 360.16 & 0.13 & 312.06 & 3600.84 & 2038 & 74455 & 534252 \\
\hline $\mathrm{r} 25106$ & 383.09 & 0.47 & 202.38 & 3601.20 & 0 & 95230 & 559077 \\
\hline $\mathrm{r} 25107$ & 375.21 & 0.51 & 182.06 & 3605.80 & 1 & 101480 & 567527 \\
\hline $\mathrm{r} 25108$ & 338.26 & 0.50 & 167.97 & 3601.23 & 0 & 106755 & 574002 \\
\hline $\mathrm{r} 25109$ & 370.95 & 0.42 & 216.49 & 3602.69 & 8 & 85730 & 548277 \\
\hline $\mathrm{r} 25110$ & 338.21 & 0.48 & 175.23 & 3602.76 & 0 & 99880 & 565352 \\
\hline $\mathrm{r} 25111$ & 359.79 & 0.49 & 182.06 & 3605.31 & 1 & 99780 & 565227 \\
\hline $\mathrm{r} 25112$ & 293.79 & 0.38 & 180.81 & 3610.35 & 80 & 109505 & 577977 \\
\hline $\mathrm{r} 25201$ & 324.10 & 0.09 & 294.26 & 3601.18 & 55390 & 77730 & 539977 \\
\hline $\mathrm{r} 25202$ & 371.44 & 0.46 & 202.38 & 3603.45 & 1 & 97280 & 562552 \\
\hline $\mathrm{r} 25203$ & 351.78 & 0.48 & 182.06 & 3600.60 & 0 & 102705 & 569927 \\
\hline $\mathrm{r} 25205$ & 316.87 & 0.47 & 167.97 & 3601.22 & 0 & 107530 & 575527 \\
\hline $\mathrm{r} 25206$ & 350.16 & 0.40 & 209.62 & 3603.63 & 768 & 88930 & 553452 \\
\hline $\mathrm{r} 25207$ & 311.37 & 0.46 & 178.29 & 3606.81 & 112 & 101480 & 567927 \\
\hline $\mathrm{r} 25208$ & 280.72 & 0.46 & 168.76 & 3608.59 & 213 & 104605 & 572152 \\
\hline $\mathrm{r} 25209$ & 317.58 & 0.39 & 198.64 & 3605.08 & 4 & 108055 & 576152 \\
\hline $\mathrm{r} 25210$ & 364.39 & 0.50 & 182.06 & 3605.46 & 144 & 96780 & 562727 \\
\hline $\mathrm{r} 25211$ & 278.00 & 0.39 & 167.97 & 3601.34 & 0 & 99230 & 565552 \\
\hline
\end{tabular}


Table 42 - Results for the class of instances R, with $150 \%$ drone speed and $75 \%$ drone eligible weight, using the HGA as a starting solution.

\begin{tabular}{c|c|c|c|c|c|c|c}
\hline Instance & $\begin{array}{c}\text { Objective } \\
\text { Function }\end{array}$ & GAP & Dual & Exec. Time & Nodes & Variables & Constraints \\
\hline $\mathrm{r} 25101$ & 272.16 & 0.14 & 232.76 & 3603.55 & 85686 & 73430 & 537377 \\
\hline $\mathrm{r} 25102$ & 407.35 & 0.70 & 122.45 & 3603.19 & 0 & 106505 & 575252 \\
\hline $\mathrm{r} 25103$ & 374.28 & 0.69 & 115.21 & 3604.90 & 1 & 118555 & 590077 \\
\hline $\mathrm{r} 25104$ & 340.41 & 0.68 & 107.97 & 3602.05 & 0 & 127180 & 600077 \\
\hline $\mathrm{r} 25105$ & 382.35 & 0.55 & 172.43 & 3601.58 & 1 & 88580 & 554502 \\
\hline $\mathrm{r} 25106$ & 378.47 & 0.68 & 122.45 & 3604.93 & 2 & 112480 & 582452 \\
\hline $\mathrm{r} 25107$ & 365.45 & 0.68 & 115.21 & 3601.68 & 0 & 121030 & 593202 \\
\hline $\mathrm{r} 25108$ & 334.38 & 0.68 & 107.97 & 3601.68 & 0 & 128105 & 601477 \\
\hline $\mathrm{r} 25109$ & 366.29 & 0.64 & 132.21 & 3600.85 & 0 & 103280 & 571952 \\
\hline $\mathrm{r} 25110$ & 334.61 & 0.67 & 110.98 & 3601.19 & 0 & 120005 & 591602 \\
\hline $\mathrm{r} 25111$ & 336.38 & 0.66 & 115.21 & 3602.06 & 0 & 119305 & 590877 \\
\hline $\mathrm{r} 25112$ & 258.47 & 0.59 & 106.34 & 3601.77 & 0 & 131080 & 605677 \\
\hline $\mathrm{r} 25201$ & 442.21 & 0.61 & 170.96 & 3600.44 & 17281 & 90730 & 559102 \\
\hline $\mathrm{r} 25202$ & 356.26 & 0.66 & 122.45 & 3603.29 & 1 & 114380 & 585777 \\
\hline $\mathrm{r} 25203$ & 350.88 & 0.67 & 115.21 & 3600.98 & 0 & 122380 & 595727 \\
\hline $\mathrm{r} 25204$ & 301.11 & 0.64 & 107.97 & 3607.22 & 1 & 128905 & 603027 \\
\hline $\mathrm{r} 25205$ & 346.34 & 0.62 & 132.21 & 3602.69 & 2 & 106905 & 577552 \\
\hline $\mathrm{r} 25206$ & 308.96 & 0.65 & 107.97 & 3604.85 & 1 & 120455 & 593027 \\
\hline $\mathrm{r} 25207$ & 304.01 & 0.64 & 107.97 & 3601.94 & 0 & 124755 & 598427 \\
\hline $\mathrm{r} 25208$ & 265.33 & 0.59 & 107.97 & 3601.73 & 0 & 129430 & 603652 \\
\hline $\mathrm{r} 25209$ & 328.76 & 0.65 & 115.24 & 3604.31 & 1 & 115580 & 587652 \\
\hline $\mathrm{r} 25210$ & 367.06 & 0.69 & 115.21 & 3604.17 & 2 & 118380 & 590827 \\
\hline $\mathrm{r} 25211$ & 275.77 & 0.61 & 106.34 & 3601.44 & 0 & 131105 & 605727 \\
\hline
\end{tabular}

Table 43 - Results for the class of instances RC, with 50\% drone speed and 25\% drone eligible weight, using the HGA as a starting solution.

\begin{tabular}{c|c|c|c|c|c|c|c}
\hline Instance & $\begin{array}{c}\text { Objective } \\
\text { Function }\end{array}$ & GAP & Dual & Exec. Time & Nodes & Variables & Constraints \\
\hline rc25101 & 406.84 & 0.24 & 309.63 & 3600.39 & 148168 & 28255 & 479927 \\
\hline rc25102 & 441.77 & 0.72 & 125.54 & 3631.48 & 6964 & 33705 & 489577 \\
\hline rc25103 & 358.59 & 0.76 & 86.11 & 3608.60 & 15199 & 37855 & 496377 \\
\hline rc25104 & 303.42 & 0.72 & 84.41 & 3601.60 & 1314 & 39005 & 498677 \\
\hline rc25105 & 513.32 & 0.65 & 179.86 & 3600.43 & 11582 & 32330 & 486452 \\
\hline rc25106 & 331.78 & 0.65 & 116.39 & 3625.40 & 5699 & 32780 & 487352 \\
\hline rc25107 & 287.15 & 0.73 & 78.86 & 3601.23 & 2308 & 36930 & 494677 \\
\hline rc25108 & 287.15 & 0.73 & 78.66 & 3601.62 & 2564 & 39805 & 500127 \\
\hline rc25201 & 360.35 & 0.00 & 360.34 & 28.64 & 7943 & 31080 & 485852 \\
\hline rc25202 & 338.68 & 0.65 & 117.56 & 3614.36 & 12682 & 35630 & 493577 \\
\hline rc25203 & 326.84 & 0.74 & 86.42 & 3601.22 & 4760 & 39155 & 498977 \\
\hline rc25204 & 304.08 & 0.72 & 84.53 & 3603.37 & 1931 & 39930 & 500527 \\
\hline rc25205 & 337.40 & 0.46 & 180.72 & 3600.94 & 10644 & 35180 & 492152 \\
\hline rc25206 & 325.07 & 0.63 & 119.72 & 3604.92 & 17478 & 35255 & 492452 \\
\hline rc25207 & 295.65 & 0.70 & 88.28 & 3601.43 & 4579 & 37655 & 496577 \\
\hline rc25208 & 260.22 & 0.70 & 78.40 & 3602.07 & 702 & 40655 & 501827 \\
\hline
\end{tabular}


Table 44 - Results for the class of instances RC, with 50\% drone speed and 50\% drone eligible weight, using the HGA as a starting solution.

\begin{tabular}{l|c|c|c|c|c|c|c}
\hline Instance & $\begin{array}{c}\text { Objective } \\
\text { Function }\end{array}$ & GAP & Dual & Exec. Time & Nodes & Variables & Constraints \\
\hline rc25101 & 340.31 & 0.45 & 188.05 & 3600.66 & 41939 & 36130 & 501402 \\
\hline rc25102 & 331.04 & 0.83 & 57.14 & 3601.12 & 4 & 43830 & 513202 \\
\hline rc25103 & 306.04 & 0.88 & 38.14 & 3601.45 & 1 & 49130 & 521127 \\
\hline rc25104 & 287.27 & 0.90 & 30.04 & 3602.12 & 1 & 51030 & 524177 \\
\hline rc25105 & 450.41 & 0.72 & 124.37 & 3600.79 & 5 & 41630 & 509252 \\
\hline rc25106 & 305.81 & 0.84 & 47.26 & 3601.24 & 4 & 42355 & 510427 \\
\hline rc25107 & 276.40 & 0.88 & 31.95 & 3601.51 & 3 & 48830 & 520052 \\
\hline rc25108 & 272.51 & 0.89 & 29.95 & 3601.68 & 1 & 52355 & 526152 \\
\hline rc25201 & 348.73 & 0.23 & 267.85 & 3600.41 & 189965 & 39105 & 507477 \\
\hline rc25202 & 334.93 & 0.81 & 63.95 & 3601.93 & 258 & 45755 & 517202 \\
\hline rc25203 & 320.90 & 0.88 & 38.47 & 3601.19 & 5 & 50430 & 523727 \\
\hline rc25204 & 297.04 & 0.89 & 31.87 & 3602.26 & 2 & 51955 & 526027 \\
\hline rc25205 & 333.99 & 0.54 & 154.39 & 3601.96 & 207 & 44655 & 515127 \\
\hline rc25206 & 318.90 & 0.82 & 57.33 & 3601.57 & 1038 & 44380 & 515077 \\
\hline rc25207 & 290.32 & 0.88 & 33.74 & 3602.44 & 34 & 48905 & 521327 \\
\hline rc25208 & 252.22 & 0.88 & 29.95 & 3601.05 & 0 & 53205 & 527852 \\
\hline
\end{tabular}

Table 45 - Results for the class of instances RC, with 50\% drone speed and 75\% drone eligible weight, using the HGA as a starting solution.

\begin{tabular}{c|c|c|c|c|c|c|c}
\hline Instance & $\begin{array}{c}\text { Objective } \\
\text { Function }\end{array}$ & GAP & Dual & Exec. Time & Nodes & Variables & Constraints \\
\hline rc25101 & 450.81 & 0.66 & 154.21 & 3600.77 & 70739 & 37630 & 506577 \\
\hline rc25102 & 378.69 & 0.88 & 46.81 & 3600.45 & 0 & 46230 & 519277 \\
\hline rc25103 & 311.90 & 0.90 & 31.37 & 3601.59 & 1 & 51980 & 527652 \\
\hline rc25104 & 286.12 & 0.91 & 25.30 & 3602.26 & 2 & 54380 & 531202 \\
\hline rc25105 & 444.98 & 0.75 & 112.55 & 3600.84 & 3 & 43880 & 515177 \\
\hline rc25106 & 361.77 & 0.91 & 33.41 & 3601.23 & 3 & 44955 & 516702 \\
\hline rc25107 & 277.21 & 0.90 & 27.04 & 3601.84 & 1 & 52030 & 526927 \\
\hline rc25108 & 271.23 & 0.91 & 25.13 & 3601.79 & 3 & 55855 & 533327 \\
\hline rc25201 & 350.48 & 0.24 & 264.63 & 3600.39 & 97080 & 40605 & 512652 \\
\hline rc25202 & 334.93 & 0.80 & 65.81 & 3620.15 & 2771 & 48155 & 523277 \\
\hline rc25203 & 321.86 & 0.90 & 32.80 & 3634.34 & 62 & 53280 & 530252 \\
\hline rc25204 & 295.15 & 0.91 & 25.34 & 3602.00 & 2 & 55305 & 533052 \\
\hline rc25205 & 331.00 & 0.57 & 143.03 & 3601.58 & 648 & 46905 & 521052 \\
\hline rc25206 & 384.27 & 0.88 & 46.76 & 3601.18 & 20 & 46830 & 521202 \\
\hline rc25208 & 287.28 & 0.91 & 25.75 & 3600.70 & 0 & 51655 & 527752 \\
\hline
\end{tabular}


Table 46 - Results for the class of instances RC, with 100\% drone speed and 25\% drone eligible weight, using the HGA as a starting solution.

\begin{tabular}{c|c|c|c|c|c|c|c}
\hline Instance & $\begin{array}{c}\text { Objective } \\
\text { Function }\end{array}$ & GAP & Dual & Exec. Time & Nodes & Variables & Constraints \\
\hline rc25101 & 392.82 & 0.33 & 262.15 & 3600.78 & 45323 & 33255 & 485052 \\
\hline rc25102 & 441.16 & 0.70 & 130.28 & 3601.44 & 2530 & 39355 & 495277 \\
\hline rc25103 & 373.61 & 0.76 & 88.37 & 3601.52 & 8 & 44530 & 503102 \\
\hline rc25104 & 355.12 & 0.76 & 83.93 & 3602.57 & 425 & 45905 & 505627 \\
\hline rc25105 & 488.03 & 0.64 & 177.99 & 3600.96 & 9751 & 38155 & 492377 \\
\hline rc25106 & 394.68 & 0.71 & 113.08 & 3601.41 & 5813 & 38505 & 493152 \\
\hline rc25107 & 286.93 & 0.73 & 78.31 & 3616.08 & 1459 & 43955 & 501752 \\
\hline rc25108 & 286.93 & 0.73 & 78.66 & 3602.59 & 853 & 46780 & 507152 \\
\hline rc25201 & 336.95 & 0.14 & 289.77 & 3675.45 & 131371 & 35580 & 490527 \\
\hline rc25202 & 335.81 & 0.65 & 117.55 & 3600.70 & 12654 & 40780 & 498802 \\
\hline rc25203 & 320.39 & 0.73 & 86.58 & 3602.20 & 2121 & 45830 & 505702 \\
\hline rc25204 & 302.09 & 0.72 & 83.09 & 3602.42 & 559 & 46830 & 507477 \\
\hline rc25205 & 337.18 & 0.47 & 177.04 & 3600.67 & 17171 & 40755 & 497802 \\
\hline rc25206 & 308.70 & 0.62 & 118.85 & 3600.65 & 9390 & 40930 & 498202 \\
\hline rc25207 & 291.31 & 0.69 & 90.05 & 3602.00 & 1253 & 44455 & 503427 \\
\hline rc25208 & 254.87 & 0.69 & 78.61 & 3603.17 & 575 & 47705 & 508927 \\
\hline
\end{tabular}

Table 47 - Results for the class of instances RC, with 100\% drone speed and 75\% drone eligible weight, using the HGA as a starting solution.

\begin{tabular}{|c|c|c|c|c|c|c|c|}
\hline Instance & $\begin{array}{l}\text { Objective } \\
\text { Function }\end{array}$ & GAP & Dual & Exec. Time & Nodes & Variables & Constraints \\
\hline rc25101 & 377.09 & 0.82 & 69.17 & 3601.05 & 1 & 54030 & 523027 \\
\hline $\mathrm{rc} 25102$ & 361.33 & 0.89 & 41.00 & 3600.76 & 0 & 67580 & 540677 \\
\hline rc25103 & 300.84 & 0.90 & 31.01 & 3600.11 & 0 & 75005 & 550727 \\
\hline rc25104 & 275.68 & 0.91 & 24.95 & 3603.90 & 0 & 79555 & 556427 \\
\hline $\mathrm{rc} 25105$ & 393.97 & 0.90 & 38.21 & 3600.74 & 0 & 64605 & 535977 \\
\hline rc25106 & 286.73 & 0.89 & 31.98 & 3602.17 & 1 & 65805 & 537602 \\
\hline $\mathrm{rc} 25107$ & 264.54 & 0.91 & 24.88 & 3601.27 & 0 & 77205 & 552152 \\
\hline rc25108 & 260.32 & 0.90 & 24.84 & 3600.17 & 0 & 81555 & 559077 \\
\hline rc25201 & 402.08 & 0.54 & 184.30 & 3601.11 & 1173 & 57630 & 529752 \\
\hline rc25202 & 328.37 & 0.87 & 42.61 & 3601.54 & 3 & 69355 & 544527 \\
\hline rc25203 & 306.59 & 0.90 & 31.02 & 3602.61 & 1 & 76730 & 553752 \\
\hline rc25204 & 284.20 & 0.91 & 24.95 & 3601.24 & 0 & 80505 & 558302 \\
\hline rc25205 & 325.21 & 0.83 & 56.08 & 3601.35 & 7 & 67080 & 541277 \\
\hline rc25206 & 308.30 & 0.88 & 36.82 & 3601.51 & 1 & 67680 & 542102 \\
\hline rc25207 & 278.37 & 0.91 & 25.04 & 3601.18 & 0 & 75780 & 551927 \\
\hline rc25208 & 231.11 & 0.89 & 24.84 & 3601.37 & 0 & 82605 & 560977 \\
\hline
\end{tabular}


Table 48 - Results for the class of instances RC, with $150 \%$ drone speed and 25\% drone eligible weight, using the HGA as a starting solution.

\begin{tabular}{l|c|c|c|c|c|c|c}
\hline Instance & $\begin{array}{c}\text { Objective } \\
\text { Function }\end{array}$ & GAP & Dual & Exec. Time & Nodes & Variables & Constraints \\
\hline $\mathrm{rc} 25101$ & 450.54 & 0.43 & 256.66 & 3601.13 & 36158 & 49355 & 501177 \\
\hline $\mathrm{rc} 25102$ & 428.65 & 0.76 & 103.89 & 3601.32 & 2 & 59830 & 515777 \\
\hline $\mathrm{rc} 25103$ & 320.32 & 0.73 & 84.70 & 3604.56 & 328 & 68780 & 527352 \\
\hline $\mathrm{rc} 25104$ & 302.88 & 0.73 & 82.31 & 3605.10 & 132 & 70455 & 530177 \\
\hline $\mathrm{rc} 25105$ & 452.16 & 0.61 & 177.55 & 3602.46 & 2179 & 59530 & 513752 \\
\hline $\mathrm{rc} 25106$ & 389.78 & 0.71 & 113.08 & 3603.21 & 382 & 59730 & 514377 \\
\hline $\mathrm{rc} 25107$ & 286.93 & 0.72 & 80.46 & 3603.51 & 1 & 67905 & 525702 \\
\hline $\mathrm{rc} 25108$ & 286.93 & 0.73 & 78.31 & 3601.93 & 2 & 72255 & 532627 \\
\hline $\mathrm{rc} 25201$ & 341.14 & 0.28 & 244.11 & 3600.44 & 73539 & 52105 & 507102 \\
\hline $\mathrm{rc} 25202$ & 329.42 & 0.69 & 103.07 & 3601.99 & 390 & 61180 & 519202 \\
\hline $\mathrm{rc} 25203$ & 320.39 & 0.74 & 84.70 & 3603.19 & 3 & 70630 & 530502 \\
\hline $\mathrm{rc} 25204$ & 302.24 & 0.72 & 83.93 & 3605.03 & 436 & 71755 & 532402 \\
\hline $\mathrm{rc} 25205$ & 370.63 & 0.52 & 176.63 & 3601.92 & 3492 & 61555 & 518602 \\
\hline $\mathrm{rc} 25206$ & 308.70 & 0.62 & 117.08 & 3601.98 & 717 & 61530 & 518802 \\
\hline $\mathrm{rc} 25208$ & 291.31 & 0.70 & 87.88 & 3603.90 & 132 & 67530 & 526527 \\
\hline
\end{tabular}

Table 49 - Results for the class of instances RC, with 150\% drone speed and 50\% drone eligible weight, using the HGA as a starting solution.

\begin{tabular}{c|c|c|c|c|c|c|c}
\hline Instance & $\begin{array}{c}\text { Objective } \\
\text { Function }\end{array}$ & GAP & Dual & Exec. Time & Nodes & Variables & Constraints \\
\hline rc25101 & 365.58 & 0.63 & 136.03 & 3601.68 & 2 & 91180 & 556527 \\
\hline rc25102 & 364.03 & 0.87 & 46.94 & 3602.25 & 1 & 116055 & 585477 \\
\hline rc25103 & 294.23 & 0.87 & 37.93 & 3601.81 & 0 & 131905 & 603952 \\
\hline rc25104 & 271.78 & 0.89 & 29.54 & 3602.74 & 0 & 138130 & 611327 \\
\hline rc25105 & 368.41 & 0.88 & 45.93 & 3601.39 & 0 & 112405 & 580102 \\
\hline rc25106 & 277.57 & 0.83 & 45.93 & 3603.75 & 2 & 116155 & 584277 \\
\hline rc25107 & 263.37 & 0.89 & 29.54 & 3601.15 & 0 & 135455 & 606727 \\
\hline rc25108 & 256.03 & 0.88 & 29.54 & 3602.23 & 0 & 143130 & 616977 \\
\hline rc25201 & 378.99 & 0.66 & 127.23 & 3601.35 & 11933 & 97480 & 565952 \\
\hline rc25202 & 397.86 & 0.88 & 46.94 & 3601.94 & 0 & 118855 & 590352 \\
\hline rc25203 & 311.83 & 0.88 & 37.93 & 3602.58 & 0 & 134955 & 608302 \\
\hline rc25204 & 279.43 & 0.89 & 29.54 & 3602.05 & 0 & 140030 & 614152 \\
\hline rc25205 & 324.89 & 0.69 & 100.69 & 3602.40 & 1 & 114505 & 585027 \\
\hline rc25207 & 305.70 & 0.83 & 51.93 & 3602.40 & 2 & 114730 & 585477 \\
\hline rc25208 & 234.15 & 0.88 & 33.54 & 3601.78 & 0 & 130905 & 603377 \\
\hline
\end{tabular}


Table 50 - Results for the class of instances RC, with 150\% drone speed and 75\% drone eligible weight, using the HGA as a starting solution.

\begin{tabular}{l|c|c|c|c|c|c|c}
\hline Instance & $\begin{array}{c}\text { Objective } \\
\text { Function }\end{array}$ & GAP & Dual & Exec. Time & Nodes & Variables & Constraints \\
\hline rc25101 & 363.55 & 0.84 & 56.45 & 3601.84 & 1 & 100830 & 569852 \\
\hline rc25102 & 348.75 & 0.88 & 40.77 & 3601.22 & 0 & 130405 & 603502 \\
\hline rc25103 & 287.38 & 0.89 & 30.80 & 3602.84 & 0 & 147655 & 623377 \\
\hline rc25104 & 272.66 & 0.91 & 24.80 & 3602.77 & 0 & 155630 & 632502 \\
\hline rc25105 & 427.61 & 0.93 & 31.67 & 3603.74 & 1 & 125030 & 596402 \\
\hline rc25106 & 271.50 & 0.88 & 33.24 & 3601.54 & 0 & 130455 & 602252 \\
\hline rc25107 & 260.45 & 0.90 & 24.78 & 3602.08 & 0 & 152580 & 627527 \\
\hline rc25201 & 257.81 & 0.90 & 24.78 & 3600.41 & 0 & 161380 & 638902 \\
\hline rc25202 & 323.31 & 0.87 & 40.77 & 3601.20 & 0 & 132580 & 607752 \\
\hline rc25203 & 308.97 & 0.90 & 30.81 & 3601.99 & 0 & 150105 & 627127 \\
\hline rc25204 & 282.22 & 0.91 & 24.80 & 3602.81 & 0 & 157530 & 635327 \\
\hline rc25205 & 328.99 & 0.90 & 32.16 & 3603.04 & 1 & 127055 & 601252 \\
\hline rc25206 & 306.11 & 0.90 & 31.67 & 3601.17 & 0 & 129030 & 603452 \\
\hline rc25207 & 274.81 & 0.91 & 24.82 & 3602.18 & 0 & 146780 & 622927 \\
\hline rc25208 & 228.84 & 0.89 & 24.78 & 3603.48 & 0 & 162430 & 640802 \\
\hline
\end{tabular}


ANNEX

\section{A MIXED INTEGER LINEAR PROGRAMMING MODEL FOR THE DRONE-ASSISTED TSP}

Here we present the paper published in the Simpósio Brasileiro de Pesquisa Operacional, 2018 (COSTA et al., 2018). This work was derived from an early brainstorm on drones and logistics applications, as this real case of drone usage in Iceland (O'KANE, 2017) made us wonder if this type of application was more likely to be expanded. However, since the circumstances in which this problem is more relevant are less general, i.e. the physical constraints such as lakes, islands, mountains, etc. are not applicable to every site, we decided to follow a more general possibility of the VRPD. 


\title{
A Mixed Integer Linear Programming Model for the Drone-Assisted TSP
}

\author{
Joao Guilherme Cavalcanti Costa \\ Instituto de Ciencias Matemáticas e de Computação - Universidade de São Paulo - Brasil \\ Avenida Trabalhador São-carlense, 400 - Centro- São Carlos \\ joaogcavalcanti@usp.br \\ Allan Vinícius Silva \\ Instituto de Ciencias Matemáticas e de Computação - Universidade de São Paulo - Brasil \\ Avenida Trabalhador São-carlense, 400 - Centro- São Carlos \\ allan.vinicius.silva@usp.br \\ Matheus Augusto Fernandes de Assunção \\ Instituto de Ciencias Matemáticas e de Computação - Universidade de São Paulo - Brasil \\ Avenida Trabalhador São-carlense, 400 - Centro- São Carlos \\ matheusfernandes@usp.br \\ Maristela Oliveira dos Santos \\ Instituto de Ciencias Matemáticas e de Computação - Universidade de São Paulo - Brasil \\ Avenida Trabalhador São-carlense, 400 - Centro- São Carlos \\ marieicmc.usp.br
}

\begin{abstract}
In this article, the Drone-Assisted Traveling Salesman Problem (DATSP), a new context in which drones are used to extend a classic TSP, is explored. Drones travel unimpeded by traffic and can reach areas of difficult access, whereas other vehicles might not be able to do so. In this new paradigm, an obstacle, such as a river or a mountain, would hinder a company's delivery time to the other side of that obstacle. The drone would then fly repeatedly across it, carrying packages which are then delivered to the clients by a deliveryman. This research is motivated by a real life application, where an Icelandic company started using drones to deliver food across a bay. This operational problem inspired the DATSP, which is modeled mathematically as a Mixed Integer Linear Programming (MILP) problem in this article. Finally, instances were generated and solved with the solver GUROBI and their solutions were investigated.
\end{abstract}

KEYWORDS. Drones. Traveling Salesman Problem. Mathematical Model.

Paper topics (L\&T - Logística e Transportes) 


\section{Introduction}

Drones have increasingly become part of modern society. Many companies have sought to streamline their services using drones in the context of delivering goods to customers. Icelandic company AHA, for instance, is already using Flytrex's drones to fly over a bay in Reykjavik, cutting costs and delivery times significantly [Gilchrist, 2017], as seen in Figure 1. Zipline, a company in Silicon Valley, has teamed up with the Rwandan health department and is saving lives using drones to deliver blood and medicine to hospitals in remote regions [Simmons, 2016]. DHL, the German postal service, trialled delivery drones in combination with fixed stations between which the drones fly, saving around 20 minutes in delivery time during winter [DHL, 2016]. Amazon announced plans in 2013 to make deliveries using drones 30 minutes after customers purchase an item on their website [CBS, 2013]. Dominos is testing pizza deliveries using drones in New Zealand directly to customers [Scholz, 2016]. UPS successfully tested using drones in tandem with a truck to deliver products to clients in rural areas [Lithia, 2017].

Figure 1: A comparison between a drone's route (green) and a delivery vehicle's (red) in Reykjavik.

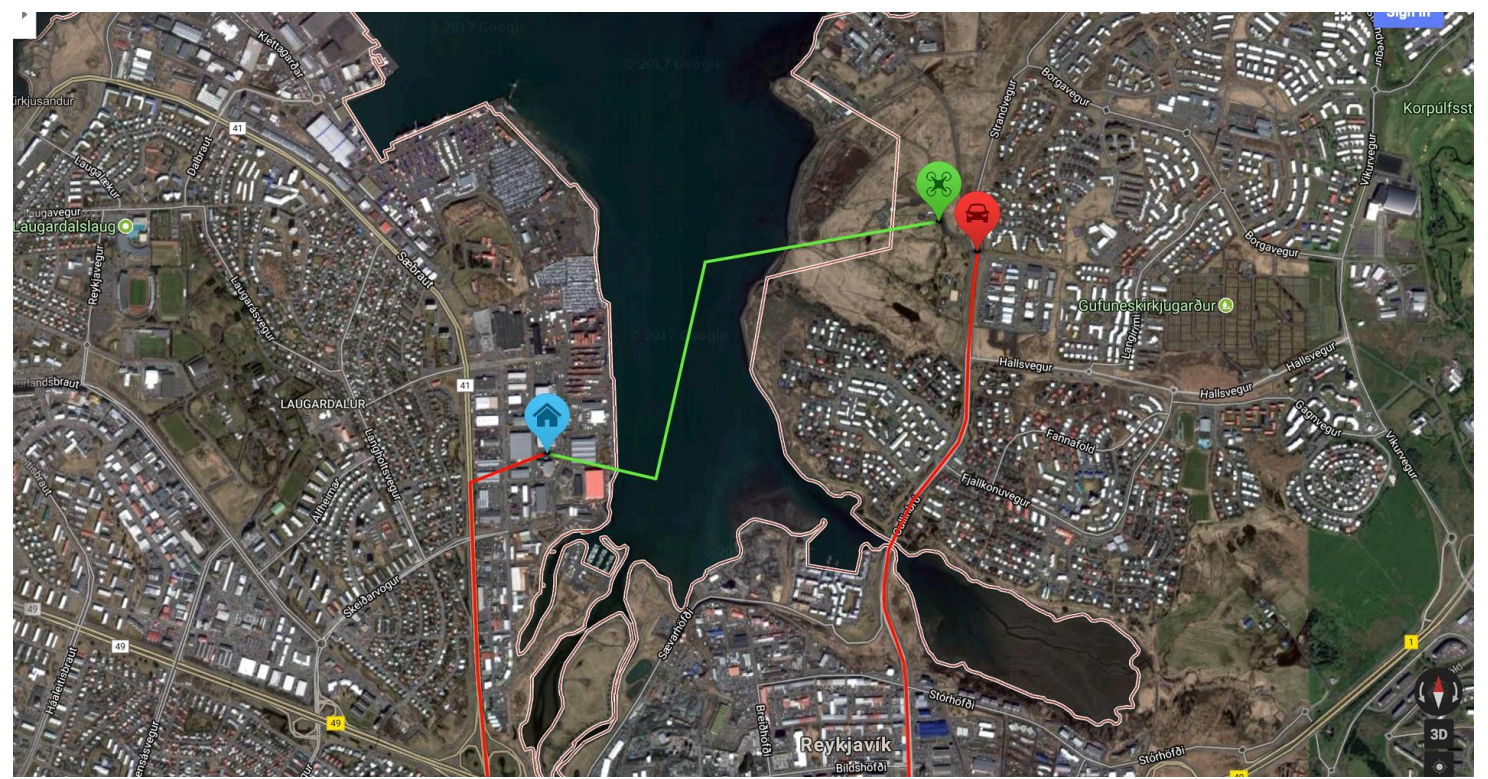

Source: Flytrex, found in [Gilchrist, 2017].

This sudden interest in this type of application is due to the advantages drones provide, such as not following traffic, being able to reach areas of difficult access, being efficient energywise [Stolaroff et al., 2018], and being greener alternatives, which could reduce greenhouse gas emissions by almost 54\% [Stolaroff and Samaras, 2018] when compared to trucks. Due to these factors, drones are already being studied in the academic field. For instance, [Murray and Chu, 2015] use a drone in tandem with a truck in the context of last-mile delivery, similarly to what has been tested by UPS. The authors propose the use of a drone to deliver to clients while the truck delivers to other clients and serves as a base for the drone, reducing the total delivery time when compared to a classic Traveling Salesman Problem (TSP) model. The work of [Dorling et al., 2017] uses drones to make deliveries within a radius around the depot on their own, allowing for multiple trips and multiple clients in the same route.

Despite the benefits granted from using drones, there are still many hindrances that need to be assessed. The issue of privacy is among the problems being discussed, since drones use cameras for their guidance systems. The risk of damage to private property or to people in case of accidents is also taken into account. The re-use of drones and the toll on battery life are also issues 
that hinder the continuous use of drones in the context of delivering goods, since modern delivery drone technology does not allow for long flight times. Moreover, modern cargo drone models have load capacities limited to only a few kilos. In spite of these issues, the authors deem pertinent the use of drones in logistics, since there are many potential benefits to using drones. Amazon's CEO, for instance, claimed in an interview that $86 \%$ of all deliveries the company makes weigh less than 3 kilos [Rose, 2015], which would more than justify their investments in drone technology.

This paper proposes and solves the Drone-Assisted Traveling Salesman Problem (DATSP), where a drone is employed to gradually transport products (such as food, medicine, or any products light enough) to a deliveryman, aiming to minimize the operational time. Unlike the other works mentioned before, the drone does not itself deliver to the customers, its function is to transport those products to the deliveryman who is then responsible for delivering them to the customers. In fact, the authors could not find a similar problem that was explored in the literature.

The rest of this paper is organized as follows: Section 2 explains the DATSP; Section 3 presents the mathematical model based on Mixed Integer Linear Programming techniques; Next, Section 4 shows the computational results obtained using the solver GUROBI. Finally, Section 5 concludes the work and presents research perspectives.

\section{The Drone-Assisted TSP}

The problem to be discussed in this section is, to the authors' best knowledge, not one that has been treated in the literature. The goal of the Drone-Assisted Traveling Salesman Problem (DATSP) is to utilize a drone to help in the delivery of goods by flying over areas of difficult access, such as rivers, lakes, bays, forests or mountains. The scenario involved comprises a drone, the obstacle, and the deliveryman waiting across from the obstacle. The drone flies over and drops off the goods to the deliveryman who delivers them to the clients on the other side. Since the total cargo to be delivered exceeds the drone's load capacity, multiple trips are necessary until every product has been delivered. After the drone unloads the products, the deliveryman executes the route and meets the drone at the drop-off point to receive a new batch of goods that needs to be delivered. The deliveryman's route is described by the solution to a TSP. Thus, the decisions of the DATSP are determining how many visits the drone makes, which client is served in which visit, and the deliveryman's routes, minimizing total operational time. A similar mode of operation being used by Icelandic company AHA demonstrates that using drones to help deliveries manages to reduce time and costs to the company [Gilchrist, 2017].

Figure 2 illustrates the DATSP, where two landmasses separated by a body of water can only be crossed using a boat or a ferry (there is no bridge nearby). On one side there is a company that delivers products locally. On the other side there are customers to whom delivering products might not be feasible without an alternative mode of transportation. The presence of an obstacle hence limits the number of possible customers to that company or discourages clients from using their services. Therefore, a drone can fly across the water and the deliveryman waiting on the other side can deliver to those customers. In the example, the route shown visits both customers with a green mark, whereas the other ones will be served during later visits.

Companies that deliver food, medicine or other light objects would benefit from this type of application by increasing their client base, reducing delivery times, improving market competitiveness and customer satisfaction. Given that this type of delivery is time-sensitive, the DATSP model could be used to schedule deliveries in short periods of time. For instance, in the context of food delivery, the customer does not expect to wait more than two hours, thus a possible strategy could be to solve the DATSP hourly or whenever a certain amount of orders is placed.

\section{The Mathematical Model for the DATSP}

The problem can be represented by a graph in which $V=\{0,1, \ldots, n\}$ is the set of vertices (0 being the drone's drop-off point) and $E=\{(i, j) \mid i, j \in V\}$ is the arcs set, in which $(i, j)$ is an 
Figure 2: An example of a DATSP application: A company utilizes the drone to cross the river.

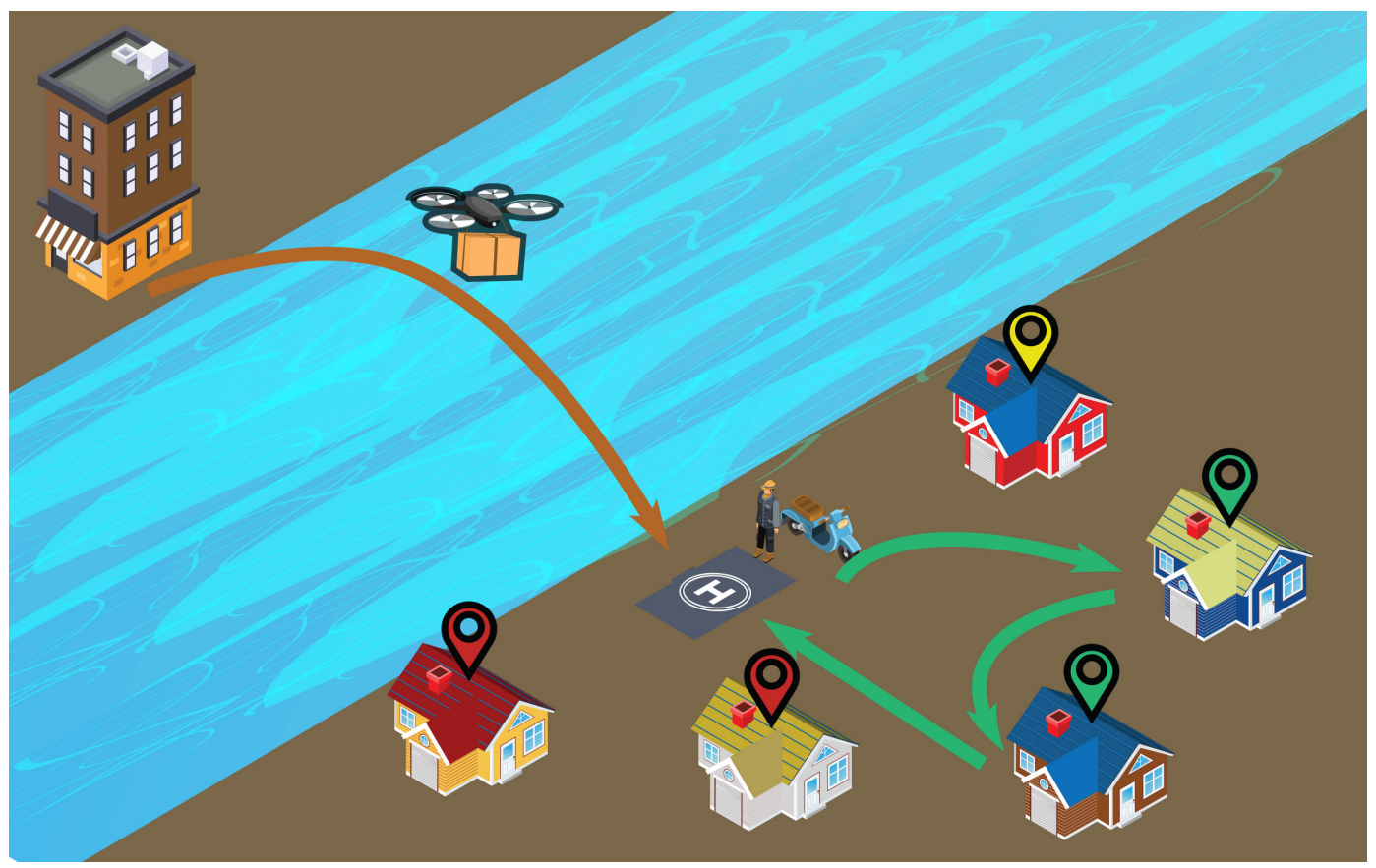

Source: Authors, based on "Designed by Freepik" artwork.

arc that represents the deliveryman's transit from $i$ to $j$. Each client $i$ also has a demand that must be served by the deliveryman.

The main decisions of the DATSP are determining the number of trips the drone makes, the clients that deliveryman serves after each trip and the routes the deliveryman travels, aiming to minimize the total operational time. All demands are known at the start of the operation, and, since the total weight of the demands exceeds the drone's load capacity, many trips between the depot and the drone's drop-off point are necessary. For every time the drone unloads goods at the drop-off point, the route that must be executed by the deliveryman must be determined in a way that the deliveryman will always return to the drop-off point before the drone arrives with more products to be delivered. There is no priority between clients. The mathematical model for the DATSP is presented with its parameters and variables as follows.

\section{Parameters:}

$V: \quad$ vertex set;

$C: \quad$ client set $(C=V \backslash\{0\})$;

$M: \quad$ set of the possible number of times the drone unloads at the drop-off point;

$l_{i}: \quad$ the demand of client $i$;

$d_{i j}: \quad$ the distance between clients $i$ and $j$;

$Q_{d}: \quad$ the drone's load capacity;

$Q_{e}: \quad$ the deliveryman's load capacity;

$v_{d}: \quad$ the drone's average flight speed;

$v_{e}: \quad$ the deliveryman's average speed;

$\tau: \quad$ average total time the drone takes to take off and land;

$h$ : the distance between the depot and the drone's drop-off point;

$T_{\max }:$ maximum operational time;

$K: \quad$ a large enough number. 
Variables:



- Flow constraints

$$
\begin{array}{ll}
\sum_{\substack{i \in M \\
i \neq j}} p_{i m}=1, & \forall i \in C ; \\
\sum_{\substack{j \in C \\
j \in J}} x_{0 j m}=y_{m}, & \forall j \in C, \forall m \in M ; \\
\sum_{\substack{i \in V \\
i \neq j}} x_{i j m}=\sum_{\substack{i \in V \\
i \neq j}} x_{j i m}, & \forall j \in V, \forall m \in M ;
\end{array}
$$

Constraints (1) guarantee that every client is served by the end of the operation. Constraints (2) link the visit variables with the flow variables, where if a client $j$ is served on the $m$-th visit, then the deliveryman necessarily serves $j$ exactly once on that route. Constraints (3) demand that deliveries only happen in the $m$-th visit if and only if the drone makes that visit. Constraints (4) are responsible for ensuring the flow of the routes, meaning if the deliveryman arrives at client $j$ 's location on the $m$-th visit, then he must also leave $j$ on that same visit.

- Visit constraints

$$
\begin{array}{ll}
y_{m-1} \geq y_{m}, & \forall m \in M \backslash\{1\} ; \\
p_{i m} \leq s_{i m}, & \forall i \in C, \forall m \in M ; \\
s_{i,(m-1)} \leq s_{i m}, & \forall j \in C, \forall m \in M \backslash\{1\} ; \\
\sum_{i \in C}\left(1-s_{i,(m-1)}\right) \leq|C| y_{m}, & \forall m \in M \backslash\{1\} ; \\
\sum_{i \in C}\left(1-s_{i,(m-1)}\right) \geq y_{m}, & \forall m \in M \backslash\{1\} ;
\end{array}
$$


Constraints (5) guarantee that the $m$-th visit will happen if and only if the previous visit occurs as well. Constraints (6) link the variable that determines if client $i$ is served on visit $m, p_{i m}$, to the variable that memorizes the clients that have already been served, $s_{i m}$. For instance, if client 4 is served on visit number $3, p_{4,3}=1$ and, therefore, $s_{4,3}=1$. Constraints (7) ensure that, if client $i$ has already been served, then this information is passed on to future visits. Constraints (8) and (9) establish the drone's stopping point, that is, if all the clients have already been served on the $m$-th visit, then there are no more visits from the drone.

- Load constraints

$$
\begin{array}{ll}
q_{j} \leq q_{i}+l_{j}+K\left(1-\sum_{m \in M} x_{i j m}\right), & \forall i \in V, j \in C, i \neq j ; \\
q_{j} \geq q_{i}+l_{j}-K\left(1-\sum_{m \in M} x_{i j m}\right), & \forall i \in V, j \in C, i \neq j ; \\
l_{i} \leq q_{i} \leq \min \left\{Q_{e}, Q_{d}\right\}, & \forall i \in C ; \\
q_{0}=0 ; &
\end{array}
$$

Constraints (10) and (11) determine the load that has already been delivered by the deliveryman after serving client $j$, preceded by client $i$, if the route goes from $i$ to $j$. Constraints (12) guarantee that the load capacity will never be exceeded. Constraint (13) indicates the total cargo delivered at the start of every route.

- Time constraints

$$
\begin{array}{ll}
t_{j} \leq t_{i}+\frac{d_{i j}}{v_{e}}+K\left(1-\sum_{m \in M} x_{i j m}\right), & \forall i \in C, j \in C, i \neq j ; \\
t_{j} \geq t_{i}+\frac{d_{i j}}{v_{e}}-K\left(1-\sum_{m \in M} x_{i j m}\right), & \forall i \in C, j \in C, i \neq j ; \\
t_{j} \leq \gamma_{m}+\frac{d_{0 j}}{v_{e}}+K\left(1-x_{0 j m}\right), & \forall j \in C, \forall m \in M ; \\
t_{j} \geq \gamma_{m}+\frac{d_{0 j}}{v_{e}}-K\left(1-x_{0 j m}\right), & \forall j \in C, \forall m \in M ;
\end{array}
$$

Constraints (14) and (15) refer to the delivery times, meaning the time when the deliveryman starts serving client $j$, if he serves client $i$ before, is the time when he arrived on client $i$ plus the time to travel between $i$ and $j$. Constraints (16) and (17) refer to the times of the first deliveries of each route, in which the first client to be served in the $m$-th visit depends on the time the drone arrives at the drop-off point, and the time to travel from the drop-off point to client $j$.

- Time constraints for the last deliveries in each route

$$
\begin{array}{ll}
a_{i m} \leq t_{i}+\frac{d_{i 0}}{v_{e}}+K\left(1-x_{i 0 m}\right), & \forall i \in C, \forall m \in M ; \\
a_{i m} \geq t_{i}+\frac{d_{i 0}}{v_{e}}-K\left(1-x_{i 0 m}\right), & \forall i \in C, \forall m \in M ; \\
a_{i m} \leq K x_{i 0 m}, & \forall i \in C, \forall m \in M ; \\
T \geq a_{i m}, & \forall i \in C, \forall m \in M ; \\
T \leq T_{\text {max }} ; &
\end{array}
$$


Constraints (18) and (19) determine the time when the deliveryman returns to the drone's drop-off point in each visit $m$, similarly to constraints (14) and (15). Constraints (20) guarantee that the time $a_{i m}$ will be zero if $i$ is not the last client to be served in the $m$-th visit. Constraints (21) define the floor for the time of last delivery $T$. Constraint (22) limits the time for the last delivery to the maximum delivery time.

- The drone's time constraints

$$
\begin{aligned}
& \gamma_{m} \geq 2\left(\tau+\frac{h}{v_{d}}\right)+\gamma_{m-1}-K\left(1-y_{m}\right), \quad \forall m \in M \backslash\{1\} ; \\
& \gamma_{1}=\tau+\frac{h}{v_{d}}
\end{aligned}
$$

Constraints (23) determine the minimum intervals between each of the drone's visits, which are the time to travel back and forth between the depot and the drop-off point if the $m$-th visit happens. Constraint (24) establishes the time of the first visit.

- Constraints synchronizing the drone and the deliveryman

$$
\begin{array}{ll}
a_{i m} \geq \gamma_{m}-K\left(1-x_{i 0 m}\right), & \forall i \in C, \forall m \in M ; \\
a_{i(m-1)} \leq \gamma_{m}+K\left(1-x_{i 0(m-1)}\right) & \forall i \in C, \forall m \in M \backslash\{1\} ;
\end{array}
$$

Constraints (25) ensure that the deliveryman's $m$-th route ends after the drone's $m$-th visit, if visit $m$ happens. Constraints (26) guarantee that the deliveryman will be at the drone's drop-off point when the drone arrives.

$$
\begin{array}{ll}
x_{i j m} \in\{0,1\}, & \forall i \in V, \forall j \in V, \forall m \in M ; \\
p_{i m} \in\{0,1\}, & \forall i \in V, \forall m \in M ; \\
s_{i m} \in\{0,1\}, & \forall i \in V, \forall m \in M ; \\
y_{m} \in\{0,1\}, & \forall m \in M ; \\
t_{i} \geq 0, & \forall i \in C ; \\
a_{i m} \geq 0, & \forall i \in C, \forall m \in M ; \\
\gamma_{m} \geq 0, & \forall m \in M .
\end{array}
$$

The domain of the variables is described in (27) - (33)

- Objective function

\section{Minimize $T$}

The objective function (34) aims to minimize the total operational time $T$. Thus, the model for the DATSP can be written as such:

Minimize $T$

subject to: $(1)-(33)$ 


\section{Computational experiments}

In this section the computational experiments and their results are presented.

\subsection{Test environment}

The model was implemented using the programming language Python v.2.7.3 and the results were obtained using the optimization solver GUROBI v.7.0.1. The tests were executed in a computer with an Intel(R) Xeon(R) CPU E5-2620 0 @ 2.00GHz processor and 70 GB of RAM in a Linux Ubuntu v.3.5.0 x64 operating system.

\subsection{Test instances data}

Five test instances were developed for the problem, containing 4, 9, 16, 25 and 32 customers (see Table 2), randomly distributed on a $10 \mathrm{~km}$ by $10 \mathrm{~km}$ field, where the depot is located at the bottom-left corner. The demands of these clients were generated randomly between 1,2 , and 3 units of weight, and the load capacities were of 4 units of weight for the drone and 5 units of weight for the deliveryman. The average speeds were defined as $60 \mathrm{~km} / \mathrm{h}$ for the drone and $40 \mathrm{~km} / \mathrm{h}$ for the deliveryman. The distance between the depot and the drone's drop-off point is $2 \mathrm{~km}$, while the time for the drone to take off and land was defined as 1 minute. Moreover, $M$ was defined as a set $\{1, \ldots, n\}$, in which $n$ is the number of clients in a given instance, since $n$ is the maximum number of visits necessary to serve every client. The maximum operational time was defined as $T_{\max }=30 h$. The values considered for $K$ in each constraint are described in Table 1 .

Table 1: Calculation of the value given to $\mathrm{K}$ in each constraint.

\begin{tabular}{lc|l}
\hline No. & Constraint & Value \\
\hline$(10)$ & $q_{j} \leq q_{i}+l_{j}+K\left(1-\sum_{m \in M} x_{i j m}\right)$ & $K=\min \{Q e, Q d\}$ \\
\hline$(11)$ & $q_{j} \geq q_{i}+l_{j}-K\left(1-\sum_{m \in M} x_{i j m}\right)$ & $K=\min \{Q e, Q d\}+\max _{i \in C}\left\{l_{i}\right\}$ \\
\hline$(14)$ & $t_{j} \leq t_{i}+\frac{d_{i j}}{v_{e}}+K\left(1-\sum_{m \in M} x_{i j m}\right)$ & $K=T_{\max }$ \\
\hline$(15)$ & $t_{j} \geq t_{i}+\frac{d_{i j}}{v_{e}}-K\left(1-\sum_{m \in M} x_{i j m}\right)$ & $K=T_{\max }+\max _{i, j \in C}\left\{\frac{d_{i, j}}{v_{e}}\right\}$ \\
\hline$(16)$ & $t_{j} \leq \gamma_{m}+\frac{d_{0 j}}{v_{e}}+K\left(1-x_{0 j m}\right)$ & $K=T_{\max }$ \\
\hline$(17)$ & $t_{j} \geq \gamma_{m}+\frac{d_{0 j}}{v_{e}}-K\left(1-x_{0 j m}\right)$ & $\left.K=T_{\max }+\max _{j \in C}\left\{\frac{d_{0, j}}{v_{e}}\right)\right\}$ \\
\hline$(18)$ & $a_{i m} \leq t_{i}+\frac{d_{i 0}}{v_{e}}+K\left(1-x_{i 0 m}\right)$ & $K=T_{\max }$ \\
\hline$(19)$ & $a_{i m} \geq t_{i}+\frac{d_{i 0}}{v_{e}}-K\left(1-x_{i 0 m}\right)$ & $K=T_{\max }+\max _{i \in C}\left\{\frac{d_{i, 0}}{v_{e}}\right\}$ \\
\hline$(20)$ & $a_{i m} \leq K x_{i 0 m}$ & $K=T_{\max }$ \\
\hline$(23)$ & $\gamma_{m} \geq 2\left(\tau+\frac{h}{v_{d}}\right)+\gamma_{m-1}-K\left(1-y_{m}\right)$ & $K=2\left(\tau+\frac{h}{v_{d}}\right)+T_{\max }$ \\
\hline$(25)$ & $a_{i m} \geq \gamma_{m}-K\left(1-x_{i 0 m}\right)$ & $K=T_{\max }$ \\
\hline$(26)$ & $a_{i, m-1} \leq \gamma_{m}+K\left(1-x_{i 0(m-1)}\right)$ & $K=T_{\max }$ \\
\hline
\end{tabular}

\subsection{Results}

Table 2 presents the results obtained using the model and test instances. The maximum execution time was defined as 3600 seconds. The first two columns of the table indicate the instance number and the number of clients. The solutions for each instance are in column O.F. (Objective Function). The fourth column, Bound, presents the values of the lower bounds. Column Visits 
shows how many visits the drone made to the drop-off point in each instance. In Gap the relative gap values are presented. Column Execution time displays the execution times in seconds. The last two columns present the number of variables and constraints generated in each instance.

Table 2: Results for the instances obtained using the solver GUROBI.

\begin{tabular}{|c|c|c|c|c|c|c|c|c|}
\hline \multirow[b]{2}{*}{ Inst. } & \multirow[b]{2}{*}{ Clients } & \multicolumn{5}{|c|}{ Model } & \multicolumn{2}{|c|}{ Dimensions } \\
\hline & & O.F. (h) & Bound & Visits & Gap & Execution time (s) & Variables & Constraints \\
\hline 1 & 4 & 1.04 & 1.04 & 2 & 0.00 & $<1$ & 146 & 297 \\
\hline 2 & 8 & 2.01 & 2.01 & 4 & 0.00 & 77 & 802 & 1142 \\
\hline 3 & 15 & 3.63 & 0.70 & 9 & 0.81 & 3600 & 4337 & 3928 \\
\hline 4 & 24 & 6.11 & 0.30 & 13 & 0.95 & 3600 & 16226 & 9958 \\
\hline 5 & 32 & 7.56 & 0.06 & 17 & 0.99 & 3600 & 36994 & 17630 \\
\hline
\end{tabular}

Analyzing the results obtained, it is clear that the dimensions of the problem grow immensely when the number of clients is increased. It is also worth noting that, for smaller instances, the solver found the optimal solutions quickly, but cannot do so for larger instances. One aspect that might be responsible for the poor quality of the dual bounds of the problem is the amount of constraints that include the parameter $\mathrm{K}$.

Figure 3 displays the order of events in the solution for instance 1. Firstly, the drone arrives at the drop-off point ( $\gamma_{1}$, that is, $m=1$ ), bringing with it the products for customers 2 and 3 . The deliveryman then travels a route defined by the TSP between the two, going first to client 3 (time $t_{3}$ ), and then to client 2 (time $t_{2}$ ), returning to the drop-off point at time $a_{21}$. The drone's second visit $\left(\gamma_{2}, m=2\right)$ occurs at the same time the deliveryman returns from his first route. The drone does not wait at the drop-off point, as expected. The deliveryman then serves the remaining customers, 4 (time $t_{4}$ ) and 1 (time $t_{1}$ ), returning to the drop-off point at time $a_{12}$. As there are no more clients left, the drone does not make any new trips, marking the end of the operation.

Figure 3: Timeline of events for Instance 1.

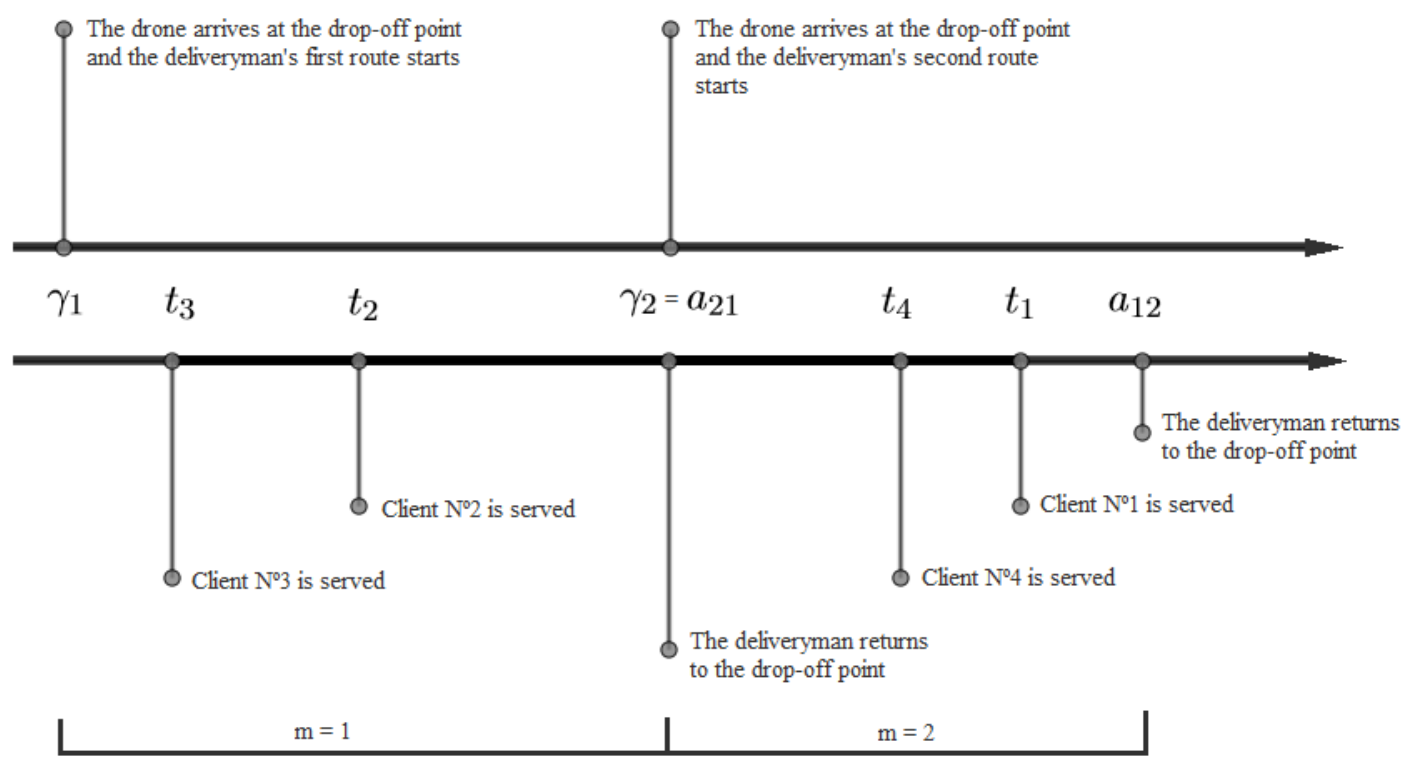

Source: Authors. 
Figure 4 shows the complete route for instance 1 in the same order as presented in Figure 3. Figure 5 illustrates the routes taken by the deliveryman during each of the drone's visits.

Figure 4: The complete route traveled by the deliveryman.

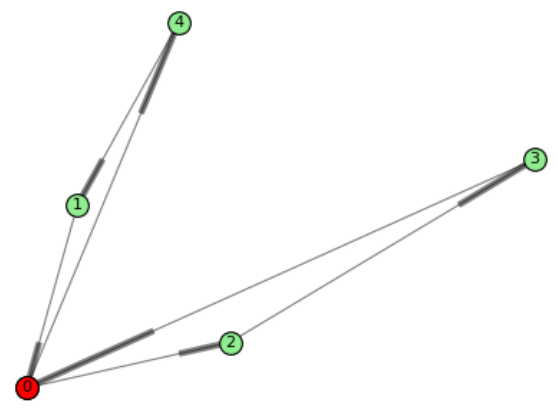

Source: Authors.

Figure 5: Individual routes taken by the deliveryman in each visit.


Source: Authors.

\section{Concluding Remarks}

In this article a preliminary study on the Drone-Assisted Traveling Salesman Problem (DATSP) was introduced. The DATSP is a new type of problem wherein a drone flies over an obstacle carrying products to a deliveryman. The authors establish that there is commercial relevance in this type of application, given that several companies have shown interest in using drones. A mathematical model elaborated as a Mixed Integer Linear Programming (MILP) problem was developed so as to represent the DATSP.

In order to test the model, five different instances were created and then solved using the solver GUROBI. The results showed that the mathematical model behaves as expected, providing optimal solutions for small instances. However, the optimal solution is not found for larger instances within the time limit, due both to the poor quality of the dual bounds and to the problem's NP-Hard nature, since the complexity of the problem grows exponentially as the number of clients increases.

Since the model does not provide optimal solutions for instances of medium and large scales, the study of heuristics or other solution methods is the starting point for any future research. Investigations concerning the mathematical model can be made so as to bring it closer to reality, as 
the current model does not consider the drone's battery consumption, which may change depending on the weight being carried, the total flight time, and even the drone's model. Another perspective could be to expand the current model for multiple drones or multiple deliverymen.

\section{Acknowledgements}

The authors would like to thank CNPq, CAPES, and CeMEAI-CEPID (FAPESP No. 2013/07375-0) for the financial support received.

\section{References}

CBS (2013). Amazon unveils futuristic plan: Delivery by drone. URL https: //www.cbsnews.com/news/amazon-unveils-futuristic-plan-deliveryby-drone/.

DHL (2016). Successful trial integration of dhl parcelcopter into logistics chain. URL http: / / www.dhl.com/en/press/releases/releases_2016/all/parcel_ecommerce/ successful_trial_integration_dhl_parcelcopter_logistics_chain.html.

Dorling, K., Heinrichs, J., Messier, G. G., and Magierowski, S. (2017). Vehicle routing problems for drone delivery. IEEE Transactions on Systems, Man, and Cybernetics: Systems, 47(1):70-85.

Gilchrist, K. (2017). World's first drone delivery service launches in iceland. URL https://www.cnbc.com/2017/08/22/worlds-first-drone-deliveryservice-launches-in-iceland.html.

Lithia, F. (2017). Ups tests residential delivery via drone launched from atop package car. URL https://www.pressroom.ups.com/pressroom/ ContentDetailsViewer.page?Concept Type=PressReleases\&id= $1487687844847-162$.

Murray, C. C. and Chu, A. G. (2015). The flying sidekick traveling salesman problem: Optimization of drone-assisted parcel delivery. Transportation Research Part C: Emerging Technologies, 54: 86-109. URL https://doi.org/10.1016/j.trc.2015.03.005.

Rose, C. (2015). Amazons jeff bezos looks to the future. URL https: / / www.cbsnews.com/ news/amazons-jeff-bezos-looks-to-the-future/.

Scholz, N. (2016). Pizza-by-drone a reality with world-first customer deliveries in new zealand. URL https://www.dominos.com.au/inside-dominos/media/november2016-pizza-by-drone-a-reality-with-world-first-customerdeliveries-in-new-zealand.

Simmons, D. (2016). Rwanda begins zipline commercial drone deliveries. URL http:// www.bbc.com/news/technology-37646474.

Stolaroff, J. and Samaras, C. (2018). Delivering packages with drones may benefit the environment. URL https://www.weforum.org/agenda/2018/02/deliveringpackages-with-drones-might-be-good-for-the-environment.

Stolaroff, J. K., Samaras, C., O’Neill, E. R., Lubers, A., Mitchell, A. S., and Ceperley, D. (2018). Energy use and life cycle greenhouse gas emissions of drones for commercial package delivery. Nature Communications, 9(1). URL https://doi.org/10.1038/s41467-017$02411-5$. 


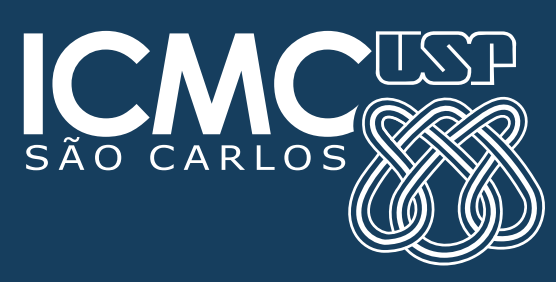

